\title{
Sparsity-Based Multipath Exploitation for Through-the-Wall Radar Imaging
}

\author{
Vom Fachbereich 18 \\ Elektrotechnik und Informationstechnik \\ der Technischen Universität Darmstadt \\ zur Erlangung der Würde eines \\ Doktor-Ingenieurs (Dr.-Ing.) \\ genehmigte Dissertation \\ von \\ Dipl.-Ing. Michael Leigsnering \\ geboren am 18. Juli 1984 in Karlsruhe
}

Referent:

Prof. Dr.-Ing. Abdelhak M. Zoubir

Korreferent:

Prof. Dr. Moeness G. Amin

Tag der Einreichung:

1. Juni 2015

Tag der mündlichen Prüfung:

28. August 2015

D 17

Darmstadt 2016 



\section{Acknowledgments}

I would like to thank all the people who have helped, inspired and supported me during my doctoral study.

First, I would like to thank Prof. Dr.-Ing. Abdelhak Zoubir for his supervision. I am very grateful for the opportunity of accomplishing my $\mathrm{PhD}$ studies in his research group and also for the great environment that I found there.

I wish to thank Prof. Dr. Moeness Amin for his supervision, guidance and exceptional hospitality when visiting Villanova University. I benefited greatly from our discussions and interactions.

Many thanks go to all the current and former colleagues at the Signal Processing Group. It has been a real pleasure to work with you all. Thanks to Dr.-Ing. Christian Debes, Dr.-Ing. Raquel Fandos, Dr.-Ing. Philipp Heidenreich, Dr.-Ing. Michael Muma, Dr.-Ing. Fiky Suratman, Dr.-Ing. Feng Yin and Dr.-Ing. Stefan Leier. Special thanks to Nevine Demitri, Sara Al-Sayed, Jürgen Hahn, Christian Weiß and Tim Schäck for proofreading and all the useful comments. I also enjoyed working together with Michael Fauss, Di Jin, Gökhan Gül, Adrian Šošić, Mark Balthasar, Wassim Suleinmann, Simon Rosenkranz and I always appreciated the support from Renate Koschella and Hauke Fath.

A special thanks to everybody at CAC at Villanova University for fruitful discussions and great hospitality - especially to Fauzia Ahmad. Thanks to Janice Moughan, Qian Jiang, Eva Lagunas, Qisong Wu and Branka Jokanovic for making my stays enjoyable.

I was happy to supervise great students whose efforts have contributed to this thesis. My sincere thanks go to Dominik Reinhard, Max Hüttenrauch and Max Stiefel.

I wish to thank my parents Karin and Franz, and other family, especially Marilena and Helmut for their unconditional love and support in all my decisions.

Finally, I am most grateful to my wife Yvonne for her love, devotion and encouragement.

Darmstadt, 1.7.2015 



\section{Kurzfassung}

In dieser Doktorarbeit werden Methoden für die Radarbildgebung durch Wände entwickelt. Ziel ist die Ausnutzung von Mehrwegausbreitung unter Verwendung der Dünnbesetztheit der Szene. Diese Art der Bildgebung macht sich das Radarprinzip zu Nutze, um verdeckte Ziele, z.B. hinter einer Wand, aufzudecken. Die gestreute elektromagnetische Welle, die von den Zielen zurückkehrt, kann den Empfänger über verschiedene Ausbreitungspfade erreichen. Dieser Effekt wird Mehrwegausbreitung genannt. Dadurch werden die Messungen mehrdeutig, was unerwünschte Geisterziele im Bild hervorruft. Daher wird Compressive Sensing (etwa komprimiertes Abtasten) angewandt, um die wahren Ziele zu rekonstruieren und Geisterziele zu unterdrücken. Dies hat den zusätzlichen Vorteil, dass weniger Messungen für die Bildrekonstruktion erforderlich sind.

Mehrwegausbreitung wird mittels additiver Signalkomponenten modelliert, welche sowohl die Rückstreuung der Ziele als auch des Gebäudes beinhalten. Dieses Modell wird in den Bildgebungsalgorithmus aufgenommen. Somit kann die zusätzliche Signalenergie, die in indirekten Reflektionen enthalten ist, ausgenutzt werden. Compressive Sensing wird eingesetzt, um Mehrwegausbreitung bei unbewegten und bewegten Zielen auszunutzen. Die Methode nutzt einerseits, dass das Bild dünnbesetzt ist. Andererseits wird die Struktur, die für Mehrwegausbreitung charakteristisch ist, ausgenutzt. Weiterhin wird untersucht, inwiefern der Dopplereffekt zusätzliche Informationen in indirekten Ausbreitungspfaden hervorruft. Darauf basierend wird ein Zweischritt-Verfahren vorgeschlagen, welches zunächst die Ziele lokalisiert. Im zweiten Schritt wird die DopplerInformation in indirekten Ausbreitungspfaden verwendet, um den Geschwindigkeitsvektor des Ziels zu schätzen. Ebenso wird eine Erweiterung auf ein Szenario mit verteilten kompakten Radarmodulen wird diskutiert. Zwei mögliche Anordnungen der Module werden betrachtet, nämlich eng zusammenstehend und weit verteilt. Der Einfluss dieser Anordnungen auf die Bildgebung wird analysiert.

Diese Arbeit beschäftigt sich Weiterhin mit nachteiligen Effekten auf die Bildgebung, welche durch die Interaktion der Welle mit dem Gebäude hervorgerufen werden. Die direkte Rückstreuung der Außenwand wird üblicherweise in der Vorverarbeitung der Messungen unterdrückt. Ein alternatives Verfahren wird vorgeschlagen, welches die Wandreflektionen und das Radarbild gemeinsam rekonstruiert. Somit wird der vorgenannte Vorverarbeitungsschritt überflüssig. Weiterhin wird der Fall betrachtet in dem kein Vorwissen über den genauen Grundriss des Gebäudes vorhanden ist. Es wird gezeigt, dass Positionsfehler der Innenwände ein Scheitern des Verfahrens zur Ausnutzung von Mehrwegausbreitung verursachen. Daher wird eine Methode vorgeschlagen, welche die Positionen der Innenwände und das Radarbild gemeinsam berechnen. Dadurch kann 
der Algorithmus auch bei unbekannten bzw. ungenau bekannten Grundrissen verwendet werden.

Die entwickelten Methoden werden anhand simulierter und gemessener Daten aus Laborexperimenten evaluiert. 


\section{Abstract}

In this $\mathrm{PhD}$ thesis sparsity-based multipath exploitation methods are developed for through-the-wall radar imaging. This imaging modality uses the radar principle to reveal targets in a scene obscured by, for example, a building wall. The scattered electromagnetic wave returning from a target may reach the receiver via different propagation paths which is called multipath. This creates ambiguities in the measurements provoking unwanted ghost targets in the image. For image reconstruction, the aforementioned issue can be resolved by utilizing the sparsity of the scene. Hence, compressive sensing is employed to recover the positions of valid targets while suppressing ghosts. As an additional benefit, fewer measurements are required for image reconstruction.

An additive multipath signal model is developed that includes returns from the targets of interest and the building structure. Incorporating the model in the image reconstruction methods allows exploitation of additional energy contained in secondary reflections. Multipath exploitation of stationary and moving targets employs compressive sensing. Therein, both sparsity and the structure originating from multipath propagation are utilized in the reconstruction problem. Moreover, the Doppler information contained in indirect propagation paths is investigated. A computationally efficient two-step approach is proposed that localizes the targets first. As a second step, the velocity vector is estimated from multipath Doppler. The scenario is extended to multiple compact radar modules, distributed around the scene. The reconstruction performance for closely-spaced and widely-separated placement is analyzed.

This work also deals with adverse effects on the imaging results caused by signal interaction with the building structure. Returns from the front wall, so-called wall clutter, are normally suppressed using a pre-processing step. A joint wall signal and target image reconstruction approach is proposed that renders prior wall clutter mitigation unnecessary. Furthermore, the case of imperfect prior knowledge of the building layout is discussed. It is shown that errors in the position of interior walls lead to complete failure of multipath exploitation. The proposed joint wall position estimation and image reconstruction procedure can deal with uncertainties in the building layout.

All proposed methods are evaluated using simulated as well as measured data from semi-controlled laboratory experiments. 



\section{Contents}

1 Introduction and Motivation 1

1.1 Motivation . . . . . . . . . . . . . . . . . . . . 1

1.2 State-of-the-Art . . . . . . . . . . . . . . . . . 2

1.3 Contributions . . . . . . . . . . . . . . . . . . 4

1.4 Publications . . . . . . . . . . . . . . . . . 5

1.5 Thesis Overview . . . . . . . . . . . . . . . . . . 7

2 Fundamentals of Compressive Sensing 9

2.1 Assumptions and Conditions for Reconstruction . . . . . . . . . . . . 10

2.1.1 Sensing on Linear Bases . . . . . . . . . . . . . . . . . . . . . . 10

2.1.2 Sparsity ....................... 11

2.1.3 Conditions on the Measurement Matrix . . . . . . . . . . . . . 12

2.2 Reconstruction Algorithms . . . . . . . . . . . . . . . . . . . . . 14

2.2.1 Optimization-Based Approaches . . . . . . . . . . . . . 14

2.2.2 Greedy Approaches . . . . . . . . . . . . . . . 15

2.3 Application to Through-the-Wall Radar Imaging . . . . . . . . . . . . . 16

3 Signal Model $\quad 19$

3.1 Ultra-Wideband Signal Model . . . . . . . . . . . . . . . . . . . 20

3.2 Stepped-Frequency Signal Model . . . . . . . . . . . . . . . . . 22

3.3 Multipath Propagation . . . . . . . . . . . . . . . . . . . 23

3.3.1 Direct Path and Wall Ringing Multipath . . . . . . . . . . . 25

3.3.2 Interior Wall Multipath . . . . . . . . . . . . . . . 27

3.3 .3 Bistatic Received Signal Model . . . . . . . . . . . . . . . . 28

3.4 Direct Wall Reflections . . . . . . . . . . . . . . . . . . . . . . 31

3.5 Efficient Sampling Schemes . . . . . . . . . . . . . . . . . . . 33

3.5.1 Ultra-Wideband Pulse Radar . . . . . . . . . . . . . . . . . 34

3.5.2 Stepped-Frequency Radar . . . . . . . . . . . . . . . 35

4 Sparsity-Based Multipath Exploitation $\quad 37$

4.1 Motivation . . . . . . . . . . . . . . . . . 37

4.2 Conventional Image Formation . . . . . . . . . . . . . . . . . . . 38

4.3 Stationary Targets . . . . . . . . . . . . . . . . . . . . . 40

4.3.1 Conventional Sparse Reconstruction . . . . . . . . . . . . . . . 41

4.3.2 Group Sparse Reconstruction . . . . . . . . . . . . . . . . . . . 41

4.3.3 Sparse Reconstruction With Overlapping Groups . . . . . . . . 44

4.3.4 Simulation and Experimental Results . . . . . . . . . . . 45 
4.4 Stationary and Moving Targets . . . . . . . . . . . . . . . . . . . . . . 49

4.4 .1 Apparent Doppler Speed . . . . . . . . . . . . . . . 49

4.4.2 Joint Target Location and Velocity Estimation . . . . . . . . . . 52

4.4.3 Target Location Reconstruction With Subsequent Velocity Estimation ...................... 53

4.4.4 Simulation and Experimental Results . . . . . . . . . . 55

4.5 Distributed Radar . . . . . . . . . . . . . . . . . . . . 64

4.5.1 Multiple Radar Unit Model . . . . . . . . . . . . . . . . . . . . 64

4.5.2 Dictionary Analysis . . . . . . . . . . . . . . . . . 66

4.5.3 Joint Group Sparse Reconstruction . . . . . . . . . . . . . . . . 68

4.5.4 Simulation Results . . . . . . . . . . . . . . . 70

4.6 Conclusions . . . . . . . . . . . . . . . . . . . . . . . . . . 74

5 Mitigating Wall Effects and Uncertainties $\quad 75$

5.1 Motivation . . . . . . . . . . . . . . . . . . . . . . . . . . . . . . . . 75

5.2 Front Wall Reflections . . . . . . . . . . . . . . . . . 77

5.2.1 Wall Reflection Model . . . . . . . . . . . . . . . . 77

5.2 .2 Separate Reconstruction . . . . . . . . . . . . . . . 79

5.2.3 Joint Group Sparse Reconstruction . . . . . . . . . . . . . . . . 79

5.2.4 Joint Overlapping Group Sparse Reconstruction . . . . . . . . . 80

5.2.5 Simulation and Experimental Results . . . . . . . . . . . . 81

5.3 Wall Location Correction . . . . . . . . . . . . . . . . . . . 85

5.3.1 Multipath Model Including Wall Position Errors . . . . . . . . . 86

5.3.2 Joint Sparse Reconstruction and Wall Position Estimation . . . 87

5.3.3 Simulation and Experimental Results . . . . . . . . . . . . . . 89

5.4 Conclusions . . . . . . . . . . . . . . . . . . . . . . . 94

6 Conclusions and Outlook $\quad 97$

6.1 Conclusions . . . . . . . . . . . . . . . . . . 97

6.1.1 Multipath Model . . . . . . . . . . . . . . . . 97

6.1.2 Sparsity-Based Multipath Exploitation . . . . . . . . . . . . 97

6.1.3 Mitigating Wall Effects and Uncertainties . . . . . . . . . . . 98

6.2 Outlook . . . . . . . . . . . . . . . . . . . . . . 99

6.2.1 Signal Model . . . . . . . . . . . . . . . . . . . . 99

6.2.2 Sparsity-Based Multipath Exploitation . . . . . . . . . . . . . 99

6.2.3 Sparse Reconstruction With Parameter Uncertainties . . . . . . 100

$\begin{array}{ll}\text { Appendix } & 101\end{array}$

A.1 Complex Amplitude Derivation . . . . . . . . . . . . . . . . . 101

A.2 Justification of the Invariance of Complex Amplitude Across the Array 102 


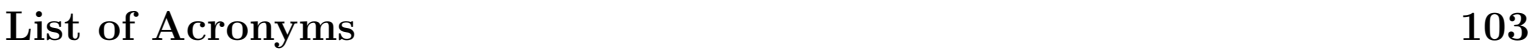

$\begin{array}{ll}\text { List of Symbols } & 105\end{array}$

$\begin{array}{ll}\text { Bibliography } & 111\end{array}$

$\begin{array}{ll}\text { Curriculum vitae } & 123\end{array}$ 



\section{Chapter 1}

\section{Introduction and Motivation}

The idea of "X-ray vision", i.e., the ability to see through walls or other visually opaque obstacles, has been popularized in many science fiction stories and comic books, most notably Superman. From a scientific perspective, real X-rays are not well-suited for this purpose as they are absorbed by walls and pass through possible objects of interest. Apart from that, ionizing radiation would be harmful for passersby. However, ElectroMagnetic (EM) waves in the radio frequency range are able to penetrate walls and may be reflected by objects beyond. This property is utilized in the emerging technology of Through-the-Wall Radar Imaging (TWRI). Employing the radar principle, i.e., transmitting and receiving EM signals, images of scenes can be obtained that cannot be accessed by optical, acoustic or thermal means. Due to its various applications, there has been a tremendous spark in related research in recent years [AAK05a, SYL05, Bur06, SS07, Bar08, DS08, DAZ09, DL09, SAA11, SM12, LAAN13a, Ami15]. It can be applied in any circumstance where an overview is desired of the situation inside a building which is impossible or too dangerous to access. This is the case in, for example, hostage crises, building fires, damaged buildings after natural disasters or detection of hidden weapon stashes. For example, the fire brigade may use a TWRI system to gain an overview of the situation. Survivors and possible ways to access them can be found without coming into contact with the fire. Hence, the safety of fire fighters need not be unnecessarily compromised.

Practical TWRI systems are limited by size, weight, power and cost constraints. These limits can be maintained by using fewer antennas and recording less data, which poses challenges to the image reconstruction procedure. Furthermore, due to the large number of scatterers in a building, e.g., walls, floor, ceiling, and furniture, the transmitted wave may reach the receiver through more than one path. This effect is called multipath propagation and causes distortions in the received radar signals. In this thesis, methods are sought that reconstruct a clear visual representation of the scene despite the harsh propagation environment and limited data.

\subsection{Motivation}

The ultimate goal of TWRI is obtaining high quality images that can be easily interpreted by a human operator or an automatic system. This requires highly resolved 
images that are free from artifacts. Moreover, widespread application of TWRI warrants compact, lightweight and affordable systems. Two major challenges need to be addressed before achieving these goals. First, high resolution images generally require radar systems with large antenna arrays and signal bandwidths. This generates large amounts of data that need to be acquired, stored, communicated and processed. Efficient sensing and reconstruction schemes are sought that require less measurements without losing information on the scene. This enables the use of shorter arrays and simpler receiver hardware. Hence, smaller, cheaper and more flexible TWRI systems may be built. Second, TWRI systems operate in a rich multipath environment. That is to say, the returns from a target may reach the receiving array via different propagation paths. This leads to ghost targets that can easily be confused with real targets [DL09]. This kind of ambiguous imaging results render the interpretation of the scene difficult. Hence, multipath should be taken into account in the image formation process.

This thesis aims at resolving the two issues above in the following way. On the one hand, the emerging Compressive Sensing (CS) framework [CW08] is employed which enables faithful image reconstruction with fewer samples and/or array elements. This is achieved by leveraging the sparsity of the scene: Since few targets are contained in a typical scene, the greater part of the image contains empty space. On the other hand, multipath is tackled by establishing a signal model that captures the characteristics of the propagation environment. Thus, the additive returns for each target can be modeled. In this way, the multipath returns can be exploited rather than considered as a nuisance. Using CS-based reconstruction, the additional energy contained in the indirect returns contributes to the amplitude of the desired targets, whereas unwanted ghost targets are eliminated. This thesis proposes various methods for sparsity-aware multipath exploitation in TWRI that reconstruct behind-the-wall targets.

\section{$1.2 \quad$ State-of-the-Art}

The roots of TWRI lie in both Ground Penetrating Radar (GPR) [DA89, Dan96] and classical airborne Synthetic Aperture Radar (SAR) [CM91]. GPR aims at detecting objects in the ground and needs to deal with a singe air-solid interface. In contrast to that, classical SAR utilizes a moving airborne radar platform to gain information on the earth's surface. Thus, free space propagation is encountered in SAR systems. TWRI draws elements from both aforementioned technologies. On the one hand, EM wave propagation through a dielectric medium is considered. On the other hand, the radar may be mounted on a moving platform, e.g., a vehicle. Various lab experiments 
and practical systems for TWRI have been developed in recent years to demonstrate performance [LN10, RCP10, SAS11, WF12, CSD14].

The field of research on TWRI is very diverse and spans disciplines from hardware design to high-level signal processing algorithms [Ami11]. The focus of this thesis lies on the processing of the radar returns and image formation. The first processing step in conventional TWRI is so-called wall clutter mitigation or wall removal suppressing the direct returns from the outer building walls. A common approach is background subtraction [AA08a, MKA ${ }^{+} 08$ ] where one assumes the availability of background data that can be coherently subtracted from the measurements of the populated scene. Other methods employ a wall model for subtraction [DS08] or use a spatial filtering approach [YA09, DWZA10]. Furthermore, subspace-based methods have been proposed [TBA11]. As a second step, an image of the scene is computed from the radar returns. This includes Delay-and-Sum Beamforming (DSBF) techniques [AAK05a, AA08a], inverse scattering approaches [SS07, LZL10, ZH11] and methods taking EM polarization into account [ZHTA11]. Multipath has been identified as a serious issue in TWRI with strong impact on the quality of the imaging results [DL09]. Early attempts aimed at mitigating the effects caused by multipath propagation [AA08a, TS10]. However, using an accurate propagation model, the additional information on the scene contained in multipath can be exploited. This information can be used to reveal parts of the target that are not illuminated by the transmitter [KSS11]. Inverse scattering-based methods exploiting multipath propagation models have been proposed [Bur09, Cha11, GS13]. Alternatively, multipath ghosts in the image can be exploited to improve the amplitude of the desired targets in the scene [SAA11, SAN13]. The time-reversal method is conceptually different in that it uses a background measurement to obtain knowledge about the scattering environment and re-transmits the processed waveform [JM09, LZL10, ZHL10].

Recently, sparsity-based methods have been introduced to TWRI research [Ami15]. CS was first applied to TWRI in [YA08]. Other sparsity-based methods revealing stationary targets behind walls were proposed in the sequel [HQWF10, LDZ11, SSA12]. Target motion was also considered in further work [AA13, QAA13, DK14]. Wall clutter mitigation was brought to the CS domain in [LAAN12, LAAN13a, AQA15]. This was extended to joint wall signal reconstruction and target imaging in [AA12a] and the work of this thesis, more specifically [LAAZ13b, LAAZ14b]. In order to achieve the full benefits of CS and multipath exploitation, the two methods were combined. Apart from the author's work [LAAZ13a, LAAZ14b, LAAZ14c, LAAZ15a], this was attempted in [GCS13, ML13]. In [GCS13], sparsifying regularization of the linear inverse scattering problem was proposed assuming full knowledge of the room geometry. Multipath elimination without prior knowledge was proposed in [ML13], however, the 
assumptions on the signal model may not hold in practice. Hence, sparsity-based multipath exploitation methods are sought that cope with limited prior knowledge of the room layout. To the best of knowledge, this has only been attempted in the author's work [LAAZ15d, LAAZ14b, LAAZ15c] For lightweight and flexible imaging systems, distributed radar units with small arrays are desired. To the best of knowledge, sparse image reconstruction for distributed TWRI was considered only in [LB15] and $\left[\mathrm{SLA}^{+} 15\right]$.

\subsection{Contributions}

The contributions of this thesis are as follows:

\section{- Multipath Model:}

A linear additive multipath propagation model is constructed. Both reflections at interior walls and within the front wall of the building are considered. This enables the exploitation of multipath in the CS framework. The model is also extended to the cases of multiple distributed radar units and unknown locations of the interior walls.

\section{- Multipath exploitation using sparse reconstruction:}

The propagation model is utilized to develop a sparsity-based image reconstruction method exploiting multipath returns. The approach is extended to deal with stationary and moving targets. Moreover, a computationally efficient method to estimate target velocity from multipath returns is proposed. Performance bounds for distributed radar configurations are analyzed. In addition, reconstruction algorithms for this case are developed.

\section{- Joint wall mitigation and target reconstruction:}

Signal reconstruction methods are proposed that simultaneously recover wall and target returns. Specially matched signal models and regularization terms are developed to obtain maximum separation and wall-clutter-free target images.

\section{- Multipath exploitation with inaccurate wall positions:}

The effect of uncertainties in the room layout on multipath exploitation is studied. A nonlinear joint wall position estimation and image reconstruction problem is formulated. Various computationally feasible sub-optimal solutions are proposed. 


\subsection{Publications}

The following publications have been produced during the period of doctoral candidacy.

\section{Book Chapter}

- M. Leigsnering and A. M. Zoubir, Compressive Sensing for Urban Radar. Boca Raton, FL: CRC Press, Aug. 2014, ch. Compressive Sensing for Urban Multipath Exploitation, pp. 153-196

\section{Internationally Refereed Journal Articles}

- M. Leigsnering, F. Ahmad, M. G. Amin, and A. Zoubir, "Compressive sensing based multipath exploitation for stationary and moving indoor target localization," IEEE Journal of Selected Topics in Signal Processing, vol. PP, no. 99, p. 1, Aug. 2015

- M. Leigsnering, F. Ahmad, M. G. Amin, and A. Zoubir, "Parametric dictionary learning for sparsity-based TWRI in multipath environments," IEEE Transactions on Aerospace and Electronic Systems, 2015, accepted

- M. Leigsnering, M. G. Amin, F. Ahmad, and A. M. Zoubir, "Multipath exploitation and suppression for SAR imaging of building interiors: An overview of recent advances," IEEE Signal Processing Magazine, vol. 31, no. 4, pp. 110-119, Jul. 2014

- M. Leigsnering, F. Ahmad, M. G. Amin, and A. Zoubir, "Multipath exploitation in through-the-wall radar imaging using sparse reconstruction," IEEE Transactions on Aerospace and Electronic Systems, vol. 50, no. 2, pp. 920-939, Apr. 2014

\section{Internationally Refereed Conference Papers}

- M. Leigsnering, F. Ahmad, M. G. Amin, and A. Zoubir, "Multipath-aware velocity estimation for sparsity-based through-the-wall radar imaging," in IEEE International Radar Conference, Arlington, VA, May 2015 
- M. Leigsnering, F. Ahmad, M. G. Amin, and A. Zoubir, "Multipath exploitation in sparse scene recovery using sensing-through-wall distributed radar sensor configurations," in IEEE International Conference on Acoustics, Speech, and Signal Processing (ICASSP), Brisbane, Australia, Apr. 2015

- M. Stiefel, M. Leigsnering, F. Ahmad, M. G. Amin, and A. M. Zoubir, "Distributed greedy sparse recovery for through-the-wall radar imaging," in International Review of Progress in Applied Computational Electromagnetics (ACES), vol. S07 Compressive Sensing, Williamsburg, VA, Mar. 2015

- M. Leigsnering, F. Ahmad, M. G. Amin, and A. Zoubir, "CS based specular multipath exploitation in TWRI under wall position uncertainties," in IEEE Sensor Array and Multichannel Signal Processing Workshop (SAM), A Coruña, Spain, Jun. 2014, pp. 481-484

- M. Leigsnering, F. Ahmad, M. G. Amin, and A. Zoubir, "Specular multipath exploitation for improved velocity estimation in through-the-wall radar imaging," in IEEE International Conference on Acoustics, Speech, and Signal Processing (ICASSP), Florence, Italy, May 2014, pp. 1060-1064

- M. Leigsnering, F. Ahmad, M. G. Amin, and A. Zoubir, "General MIMO framework for multipath exploitation in through-the-wall radar imaging," in International Workshop on Compressed Sensing Applied to Radar (CoSeRa), Bonn, Germany, Sep. 2013

- M. Leigsnering, F. Ahmad, M. G. Amin, and A. Zoubir, "Compressive sensing based specular multipath exploitation for through-the-wall radar imaging," in IEEE International Conference on Acoustics, Speech, and Signal Processing (ICASSP), Vancouver, Canada, May 2013, pp. 6004-6008

- M. Leigsnering, F. Ahmad, M. G. Amin, and A. Zoubir, "CS based wall ringing and reverberation mitigation for through-the-wall radar imaging," in IEEE Radar Conference (RADAR), Ottawa, Canada, Apr. 2013, pp. 1-5

- M. Balthasar, M. Leigsnering, and A. Zoubir, "Compressive classification for through-the-wall radar imaging," in European Signal Processing Conference (EUSIPCO), Bucharest, Romania, Aug. 2012, pp. 2288-2292

- M. Leigsnering, A. Zoubir, and M. Ghogho, "Fast wideband near-field imaging with URAs applied to urban sensing," in IEEE International Workshop on Computational Advances in Multi-Sensor Adaptive Processing (CAMSAP), San Juan, Puerto Rico, Dec. 2011, pp. 73-76 
- M. Leigsnering and A. Zoubir, "Fast wideband near-field imaging using the nonequispaced FFT with application to through-wall radar," in European Signal Processing Conference (EUSIPCO), Barcelona, Spain, Aug. 2011, pp. 1708-1712

- M. Leigsnering, C. Debes, and A. Zoubir, "Compressive sensing in through-thewall radar imaging," in IEEE International Conference on Acoustics, Speech and Signal Processing (ICASSP), Prague, Czech Republic, May 2011, pp. 4008-4011

\subsection{Thesis Overview}

The thesis is organized as follows. Chapter 2 briefly reviews the important concepts of CS theory. Various reconstruction algorithms and their properties are described. Furthermore, the assumptions and conditions for application to TWRI are discussed.

The signal model for TWRI systems is introduced in Chapter 3. The received signal for stationary and moving targets using Ultra-Wideband (UWB) radar and steppedfrequency radar is described. Furthermore, multipath propagation is characterized and modeled. This includes indirect paths via interior walls as well as multiple reflections within the front wall of the building. Direct wall reflections are also considered, i.e., propagation modes that interact solely with the building walls and not with the target scene. Finally, efficient sensing schemes are discussed that fully exploit the potential savings by the CS framework.

In Chapter 4, sparsity-based multipath exploitation methods for TWRI are proposed for various scenarios. Stationary scene reconstruction is considered for steppedfrequency radar. Two different joint location and velocity estimation methods are proposed for stationary and moving targets using UWB radar. Furthermore, distributed configuration of multiple radar modules is considered. The reconstruction method is extended to this scenario and performance bounds are analyzed depending on the placement of the radar modules. The proposed approaches are evaluated using simulated and measured data.

Chapter 5 deals with two particular issues related to the building walls, namely, wall clutter and wall location uncertainties. First, joint wall clutter mitigation and target image reconstruction based on CS is proposed. Second, a sparsity-based multipath exploitation method is developed which copes with uncertainties in the multipath model. To this end, a joint wall location estimation and image reconstruction method is introduced. The performance of the proposed methods is analyzed using the available data sets. 
Conclusions are drawn in Chapter 6 and an outlook for future work is given. 


\section{Chapter 2}

\section{Fundamentals of Compressive Sensing}

One of the most important concepts in signal processing is clearly the Shannon/Nyquist sampling theory [Sha49]. Its states that signals have to be sampled at twice their bandwidth to achieve perfect reconstruction. Nearly all of today's data acquisition schemes are based on this theory. However, it is known that the Nyquist rate is a sufficient, but not a necessary condition for perfect reconstruction [BCNV08]. In the recent years, a new sampling paradigm has been developed, known as Compressive Sensing (CS). It achieves perfect signal recovery from considerably less samples than required by Shannon's theory [CT05, DET06, CRT06, EM09, BCDH10].

This seeming contradiction can be resolved by looking into the nature of the sampled signals. In almost all data acquisition systems, the first stage is a high-precision, high data rate $\mathrm{A} / \mathrm{D}$-converter. Then, a following compression stage condenses the sampled signal into a data stream of much less samples. This is possible because those signals are compressible or redundant, which means they can be represented by a few coefficients without significant numerical or perceptual loss [CW08]. Image compression using JPEG [Wal91] or its successors is just one of the numerous examples. The bottom line is that usually a high number of samples are acquired and in the next step almost all of them are discarded. CS tackles this wasteful scheme by taking less samples in the first place. This change of concept is illustrated in Figure 2.1.

Systems based on CS generate fewer samples that need to capture the complete characteristics of the signal while showing the least possible redundancy. The sampling process is a linear operation and has to satisfy several conditions such that successful
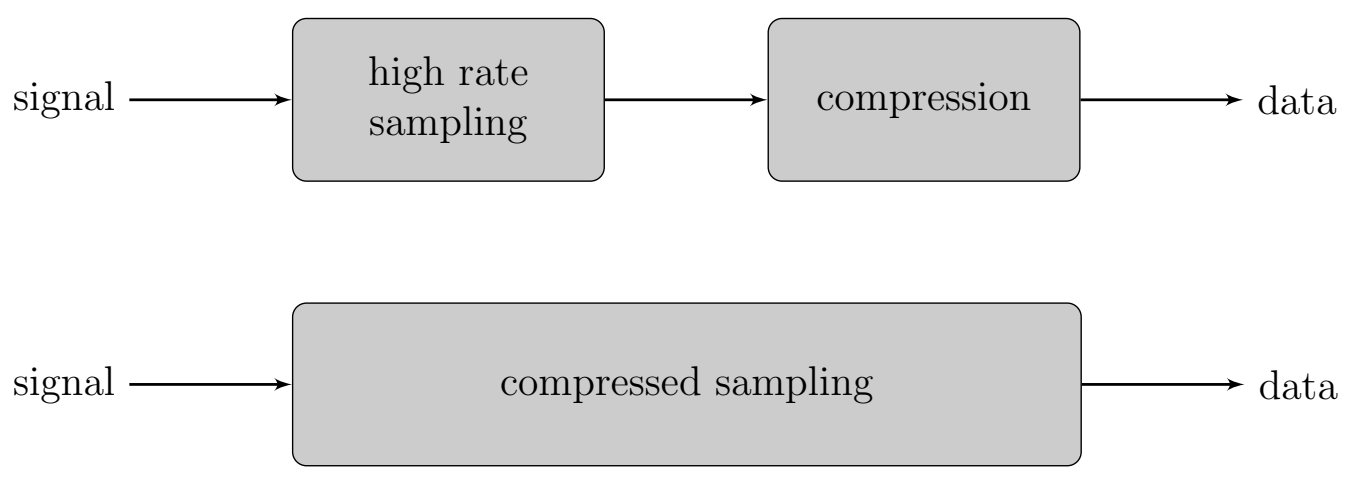

Figure 2.1. Conventional data acquisition (top) versus compressive sampling (bottom). 
reconstruction is guaranteed. A typical CS data acquisition protocol correlates the input with a set of known waveforms and samples the result [CW08]. The reconstruction of the original signal is a nonlinear operation that exploits the prior knowledge of signal structure. This can be achieved by various reconstruction algorithms as discussed later on.

Some of the basic concepts and properties of the CS framework are introduced in this chapter. Section 2.1 deals with the assumptions and conditions such that the signal can be reconstructed from the samples. Some reconstruction algorithms are discussed in Section 2.2. Finally, Section 2.3 discusses the application to Through-the-Wall Radar Imaging (TWRI). This chapter provides an overview of the most important concepts and methods in CS. For a more detailed introduction, the reader is referred to [CW08, Rom08].

\subsection{Assumptions and Conditions for Reconstruction}

This section treats the basic assumptions on the signal structure and the sensing process. Furthermore, conditions on the sensing operation that guarantee recovery of the signal from the compressed measurements are discussed.

\subsubsection{Sensing on Linear Bases}

Consider a sensing scheme where sample values $z_{i}$ from a discrete signal $\boldsymbol{r} \in \mathbb{C}^{P}$ are obtained by linear correlation with a fixed set of sequences $\phi^{(i)}$

$$
z_{i}=\left(\phi^{(i)}\right)^{T} \boldsymbol{r}, \quad i=1, \ldots, N
$$

where $N$ is the number of samples. For simplicity, the discussion is restricted to discrete signals. A continuous-time signal $r(t)$ can represented by $\boldsymbol{r}$ via sampling at the Nyquist rate. The measurement sequences $\phi^{(i)}$ could be for example sinusoids, shifted Kronecker delta functions or realizations of a random process. When the sinusoids are properly chosen, the measurement operation (2.1) is equal to taking the Discrete Fourier Transform (DFT) of $\boldsymbol{r}$ [OSB99]. Using shifted Kronecker delta functions results in a uniform or non-uniform sampling. Random sequences are frequently used in a CS setting for reasons to be explained later on. 
The linear samples can be stacked in a vector as $\boldsymbol{z}=\left[z_{1}, z_{2}, \ldots, z_{N}\right]^{T}$ resulting in a linear system of equations

$$
z=\Phi \boldsymbol{r}
$$

where $\boldsymbol{\Phi}=\left[\boldsymbol{\phi}^{(1)}, \boldsymbol{\phi}^{(2)}, \ldots, \boldsymbol{\phi}^{(N)}\right]^{T} \in \mathbb{C}^{N \times P}$. The so-called measurement matrix $\boldsymbol{\Phi}$ contains the measurement sequences in its rows. If $\boldsymbol{\Phi}$ is a non-singular square matrix, the signal can easily be recovered by multiplying the samples with the inverse. This applies, for example, if $\boldsymbol{\Phi}$ is the DFT matrix. However, CS aims at recovering the original signal from $N \ll P$ samples. Obviously, in this case, $(2.2)$ is a highly underdetermined equation system with an infinite number of solutions. Hence, additional assumptions about the signal and the measurement matrix need to be made in order to obtain a unique reconstruction result.

\subsubsection{Sparsity}

One basic assumption in CS is that the signal is sparse or can be expressed in a sparse fashion. In a sparse vector, all but few elements are zero. This means that the actual information contained in the vector is much smaller than the dimension of the vector. A vector $s$ is defined to be $\beta$-sparse if at most $\beta \in \mathbb{N}$ of its elements are nonzero.

Sparsity of a signal depends on its representation. For example, a sinusoid is not sparse in the time-domain, however, it is very sparse in the frequency domain representation. If the signal $\boldsymbol{r}$ itself is not sparse, a sparse basis or dictionary $\boldsymbol{\Psi}$ may be found. Hence, the original signal can be expressed as $\boldsymbol{r}=\boldsymbol{\Psi} \boldsymbol{s}$, where $\boldsymbol{s}$ is sparse. The overall measurements can be written by

$$
z=\Phi r=\Phi \Psi s=A s
$$

where $\boldsymbol{A}=\boldsymbol{\Phi} \boldsymbol{\Psi}$ is the sensing matrix relating the sparse coefficients to the measurements. The dictionary $\boldsymbol{\Psi}$ is not required to be a square matrix. Many man-made or natural signals have a sparse representation, when expressed in an appropriate basis [CW08]. For natural images taken by a camera, the discrete cosine transform is such a compact representation where most coefficients are zero or close to zero [Rom08]. The information is kept within a few large coefficients. Hence, the majority of the data can be discarded without noticeable deterioration of the image. This leads to a sparse signal representation.

If the support, i.e., the location of the non-zeroes of the sparse signal follows a certain structure, group sparsity or block sparsity should be considered [YL06, Kow09, EM09]. 
That means that the entries of the sparse vector form groups and the whole group is either zero or non-zero. The group pattern must be known a priori from structural knowledge of the signal. This group structure further decreases the degrees of freedom and, hence, can be exploited for signal reconstruction.

In sparse reconstruction, only the position and the value of the non-zero coefficients have to be found. Thus, the degrees of freedom are much less than the number of vector entries. By exploiting the prior knowledge of sparsity, a unique solution for the underdetermined problem (2.2) can be found. However, uniqueness depends on the amount of information extracted by the measurement as explained below.

\subsubsection{Conditions on the Measurement Matrix}

Intuitively, certain assumptions for the sensing matrix $\boldsymbol{A}$ must hold such that the signal can be reconstructed. If a significant part of the signal lies in the null space of the matrix, there is no hope in reconstructing it. Several conditions and properties for the sensing matrix have been introduced such as the Restricted Isometry Property (RIP) [CT05, CRT06], (mutual) coherence [DH01, Tro04, DET06], Null Space Property (NSP) [DH01], spark [DE03] and Uniform Uncertainty Principle (UUP) [CT06]. These conditions guarantee unique reconstruction. The coherence and the RIP are discussed in the sequel.

\section{Mutual Coherence}

For a given sensing matrix $\boldsymbol{A} \in \mathbb{C}^{N \times P}$, the coherence is defined by [Tro04]

$$
\mu(\boldsymbol{A})=\max _{i, j \neq i} \frac{\left|\boldsymbol{a}_{i}^{H} \boldsymbol{a}_{j}\right|}{\left\|\boldsymbol{a}_{i}\right\|_{2}\left\|\boldsymbol{a}_{j}\right\|_{2}},
$$

where $\boldsymbol{a}_{i}$ denotes the $i$-th column of $\boldsymbol{A}$. Hence, the coherence of a matrix is the maximum correlation between any two of its columns. The coherence should be as low as possible for successful reconstruction. In other words, if any two columns are too similar to each other, their effect on the measurement vector cannot be discerned leading to reconstruction failure.

In the case of group sparse signals, the group pattern is to be taken into account. To this end, the definition of the mutual coherence has been extended to the so-called block-coherence [EKB10]. The block-coherence is a measure of correlation between 
blocks of columns in $\boldsymbol{A}$, rather than individual columns as before. For simplicity, it is assumed that the groups occur in blocks of size $d$ in the sparse vector. The blockcoherence of a matrix $\boldsymbol{A}$ is defined as [EKB10]

$$
\mu_{\mathrm{B}}(\boldsymbol{A})=\max _{i, j \neq i} \frac{1}{d} \rho\left(\boldsymbol{A}_{i}^{H} \boldsymbol{A}_{j}\right)
$$

where $\rho(\cdot)$ is the spectral norm and $\boldsymbol{A}_{i}$ is the normalized sub-matrix of $\boldsymbol{A}$ that contains all columns belonging to the $i$-th block. Normalization of a matrix means that all columns are scaled to have unit Euclidean norm. Note that for $d=1$ the blockcoherence is equal to the regular coherence.

The advantage of mutual coherence and block-coherence is the ease of computation. For a given matrix, (2.4) or (2.5) can be calculated numerically where the cost is linear in the number of columns or blocks, respectively. Hence, performance bounds for a practical system can be found as explained in the following section. However, the theoretical results in terms of the RIP are more universal than for the coherence.

\section{Restricted Isometry Property}

The RIP for matrix $\boldsymbol{A} \in \mathbb{C}^{N \times P}$ is defined with respect to a given sparsity level $\beta$ and isometry constant $\delta_{\beta}>0$. If $\boldsymbol{A}$ obeys the RIP, the inequality

$$
\left(1-\delta_{\beta}\right)\|\boldsymbol{s}\|_{2}^{2} \leq\|\boldsymbol{A} \boldsymbol{s}\|_{2}^{2} \leq\left(1+\delta_{s}\right)\|\boldsymbol{s}\|_{2}^{2}
$$

holds for any $\beta$-sparse vector $s$ [CT05, CRT06]. For illustration, assume that the RIP holds with a small isometry constants. That means that the Euclidean norm (or length) of any $\beta$-sparse vector is merely altered by the small constant $1 \pm \delta_{\beta}$ when multiplied with $\boldsymbol{A}$. Thus, the energy of the signal is conserved. Alternatively, any set of $\beta$ columns in $\boldsymbol{A}$ is approximately linearly independent. Hence, for a $\beta$-sparse signal, the matrix behaves like an orthonormal matrix.

The issue with the RIP is, that checking the condition for a given matrix is computationally infeasible [TP14]. However, bounds can be found for certain classes of matrices, such as Gaussian random matrices. Interestingly, randomly chosen measurement vectors can be proofed to be sufficiently incoherent to obey the RIP [CW08]. Thus, correlating the measured signal with white noise sequences is an effective sensing mechanism. 


\subsection{Reconstruction Algorithms}

In this section, various reconstruction methods are reviewed. Having obtained $N \ll P$ compressed measurements $z_{i}, i=1, \ldots, N$, according to $(2.1)$ the challenging task is to recover the sparse vector, $\boldsymbol{s}$. It is a priori known that the signal is sparse. Hence, the best reconstruction $\hat{\boldsymbol{s}}$ is the sparsest vector consistent with the measurement model. This is expressed mathematically by

$$
\hat{\boldsymbol{s}}=\arg \min _{\boldsymbol{s}}\|\boldsymbol{s}\|_{0} \quad \text { s.t. } \quad \boldsymbol{z}=\boldsymbol{A s},
$$

where $\|\cdot\|_{0}$ is the $\ell_{0}$ (quasi-)norm or simply the number of non-zero elements in a vector. A unique solution follows from the RIP [CT05]. However, solving (2.7) directly is infeasible as the problem is combinatorial and NP-hard [Rom08].

Hence, tractable solutions to the recovery problem have been sought. These can be broadly distinguished into optimization-based and greedy approaches. The former category aims to solve a relaxed version of (2.7). Due to relaxation, the program belongs to the more tractable class of convex optimization problems for which efficient numerical methods are available [BV04]. The category of greedy methods builds up the support of the solution vector step-by-step until a stopping criterion is reached. The selection of the non-zero elements is locally optimal in each step, with various possible heuristics to attain a global optimum. There exists a third category, namely Bayesian CS approaches [WR04, JXC08] which is, however, not considered here.

\subsubsection{Optimization-Based Approaches}

The zero norm can be relaxed to a $\ell_{1}$-norm resulting in a tractable optimization problem. Thus, (2.7) can be recast as the so-called Basis Pursuit (BP) [CD94]

$$
\hat{\boldsymbol{s}}=\arg \min _{\boldsymbol{s}}\|\boldsymbol{s}\|_{1} \quad \text { s.t. } \quad \boldsymbol{z}=\boldsymbol{A s} .
$$

The optimization problem (2.8) can be reformulated as a linear program and, hence, efficiently solved. It has been shown that solving (2.7) and (2.8) is equivalent. However, the isometry constant of the RIP is smaller (that means stricter) for the latter [CT05].

For noisy measurements,${ }^{1}$ the closely related Basis Pursuit De-Noising (BPDN) [CRT06] is more appropriate

$$
\hat{\boldsymbol{s}}=\arg \min _{\boldsymbol{s}}\|\boldsymbol{s}\|_{1} \quad \text { s.t. } \quad\|\boldsymbol{z}-\boldsymbol{A} \boldsymbol{s}\|_{2}^{2} \leq \eta,
$$

\footnotetext{
${ }^{1}$ The author of this thesis obtained a lot of experience with noise in the last few months.
} 
where $\eta$ is a bound on the measurement errors or noise. Using Lagrangian multipliers (2.9) can be reformulated as [CDS01]

$$
\hat{\boldsymbol{s}}=\arg \min _{\boldsymbol{s}} \frac{1}{2}\|\boldsymbol{A s}-\boldsymbol{z}\|_{2}^{2}+\lambda\|\boldsymbol{s}\|_{1},
$$

where $\lambda$ is the so-called regularization parameter. As BPDN is a convex optimization problem, convergence to a global optimum is guaranteed. In a noisy scenario, measurement model (2.2) does not hold exactly due to errors in the samples. Hence, $\lambda$ controls the trade-off between fidelity to the measurements and sparsity of the solution. For $\lambda \rightarrow 0$ (2.10) approaches (2.8) but $\lambda=0$ results in the least squares solution which is not sparse. Conversely, $\lambda \rightarrow \infty$ yields an all-zero solution. In fact, it can be shown that for $\lambda>\left\|\boldsymbol{A}^{H} \boldsymbol{z}\right\|_{\infty}(2.10)$ converges to the all-zero result $\left[\mathrm{KKL}^{+} 07\right]$.

An important extension is a modified regularization term. If the $\ell_{1}$-norm is replaced by a mixed $\ell_{2} / \ell_{1}$-norm, a group sparse solution is found

$$
\hat{\boldsymbol{s}}=\arg \min _{\boldsymbol{s}} \frac{1}{2}\|\boldsymbol{A s}-\boldsymbol{z}\|_{2}^{2}+\lambda\|\boldsymbol{s}\|_{2,1} .
$$

The mixed norm is defined as

$$
\|\boldsymbol{s}\|_{2,1}=\sum_{i}\left\|\boldsymbol{s}_{g_{i}}\right\|_{2}
$$

where $g_{i}$ are index sets describing the group structure of the sparse signal. Hence, it can be seen as an outer $\ell_{1}$-norm of $\ell_{2}$-norms over the groups. Solving (2.11) requires prior knowledge on group sparsity of the signal.

Many efficient algorithms have been proposed to solve (2.10) or (2.11), for example $\left[\mathrm{KKL}^{+} 07, \mathrm{FNW07,} \mathrm{BBC} 11, \mathrm{WNF} 09, \mathrm{DYZ11}, \mathrm{YLY13}\right]$. In this thesis, mainly Sparse Reconstruction by Separable Approximation (SparSA) [WNF09] is used for sparse reconstruction. This method uses a sequence of sub-problems that can be solved efficiently. It finally converges to the optimum solution of the original problem. The method can be applied to both, complex-valued and large-scale problems, i.e., when matrix $\boldsymbol{A}$ does not fit into the memory. If the groups in the sparse signal overlap, SparSA fails. In this case, the Fast overlapping Group Lasso (FoGLasso) [YLY13] is employed. The authors follow a very similar approach as in [WNF09] but the nature of the sub-problems is different due to the overlapping groups.

\subsubsection{Greedy Approaches}

Greedy algorithms follow a different rationale by attempting to solve (2.7) directly. By using an iterative and locally optimal scheme, a good solution shall be found. 
In each step, the temporary solution is updated such that it improves with respect to a quality metric. This is repeated until a heuristic stopping criterion is reached. Greedy algorithms are usually faster than optimization-based approaches. However, the solution found may be sub-optimal and/or stricter conditions on the sparsity must be fulfilled. Well-known candidates of this class are the Orthogonal Matching Pursuit (OMP) [PRK93, TG07], the Subspace Pursuit (SP) [DM09] and Compressive Sampling Matching Pursuit (CoSaMP) [NT10]. The extension of OMP to group sparse signals has been coined Block Orthogonal Matching Pursuit (BOMP) [EKB10].

In this thesis, the BOMP is used for comparison with optimization-based approaches. In each iteration, the algorithm selects the column (or a group of columns) in $\boldsymbol{A}$ that is most correlated with the measurements. The coefficients are calculated such that they are orthogonal to the residual, i.e., the measurements minus the reconstruction estimate of the previous iteration. The norm of the residual is constantly reduced in each iteration. This process is repeated until the desired sparsity or residual energy is reached.

Reconstruction conditions that guarantee exact solutions can also be found for greedy approaches. The following conditions only hold for a noise-free environment. It has been shown that OMP perfectly recovers a $\beta$-sparse signal if [Tro04, DE03]

$$
\beta<\frac{1}{2}\left(\mu(\boldsymbol{A})^{-1}+1\right)
$$

A similar condition for block sparse reconstruction can be expressed as [EKB10]

$$
\beta<\frac{1}{2 d}\left(\mu_{\mathrm{B}}(\boldsymbol{A})^{-1}+d\right)
$$

Hence, for a given sensing matrix, the vector of interest is required to be sufficiently sparse to achieve perfect reconstruction. However, (2.13) and (2.14) are sufficient rather than necessary conditions. Therefore, the recovery process may be successful even in cases where the conditions are violated.

\subsection{Application to Through-the-Wall Radar Imaging}

When employing CS for a specific application, the above assumptions and conditions should be checked for validity. In this section, the application of CS to TWRI is considered. 
Sampling on Linear Bases: The sensing model of TWRI is discussed in the following chapter. The sensing matrix $\boldsymbol{A}$ consists of two parts. The first part is the signal propagation model that describes the physical aspects of Electro-Magnetic (EM) wave propagation, as discussed Section 3.1. After some simplifications, a linear model can be found. This part corresponds to the dictionary $\boldsymbol{\Psi}$ which is determined by the radar parameters and scene geometry. Thus, an arbitrary choice is not possible. The second part is the sampling of the received signal which can be implemented using CS. The measurement matrix $\boldsymbol{\Phi}$ corresponding to the sensing scheme can be designed to achieve desirable properties. The overall sensing matrix can be thought of as the multiplication of the two aforementioned parts.

Sparsity: The signal of interest is an image of the scene. In TWRI, we assume few targets in the room that can be approximated as point targets. Hence, targets appear as pixels with non-zero amplitude in a large image. This justifies the assumption that the image itself is sparse. After vectorization, the image can be expressed as a sparse vector $\boldsymbol{s}$. For radar systems with high resolution, a target may occupy a number of adjacent pixels. Hence, a wavelet representation of the image can be used to obtain a sparse vector [LDZ11]. Furthermore, group sparsity may arise for signals received by multiple radar units or by virtue of multipath which will be explained later on.

Conditions on the Sensing Matrix: Verifying the conditions on the sensing matrix $\boldsymbol{A}$ is difficult in TWRI. Even if the sparsity of the scene is known, it cannot be shown that the RIP holds. This is due to the fact that no computationally tractable approach exists for verifying the RIP for a given deterministic matrix [TP14]. The mutual coherence can easily be calculated for $\boldsymbol{A}$. In fact, the coherence values tend to be very high, as the columns correspond to the densely discretized pixels of the image [PEPC10]. This results in very similar measurements for two adjacent pixels. However, the mutual coherence is a sufficient but not a necessary criterion. Simulation and experimental results show that good image reconstruction performance can be achieved for highly coherent measurement matrices.

In literature, CS has been employed for TWRI successfully in various settings. In the view of efficient data acquisition, CS has been first applied to TWRI by Yoon and Amin [YA08]. Subsequently, others showed that as long as the scene is sparse, CS is able to reconstruct highly resolved images with little clutter [HQWF10, AA13, LDZ11, SSA12]. Sparse reconstruction has been examined for moving targets in the context of TWRI in [QAA13], showing similar advantages. Furthermore, sparsity-based building layout 
estimation techniques have been proposed [LAAN13b, vRdW14] that estimate wall locations.

Little work has been done on taking multipath into account in CS-based TWRI. Multipath effects cause ghost targets to appear in the image and wall reverberation artifacts. The combined effect significantly reduces the sparsity of the scene. Hence, the image cannot be sparsely represented diminishing the benefits and applicability of CS. Therefore, multipath propagation should carefully be taken into consideration when formulating the measurement model and reconstruction problem in a CS framework. The major contribution of this thesis is a CS-based formulation of the image reconstruction problem in multipath environments. 


\section{Chapter 3}

\section{Signal Model}

In this chapter, a forward scattering model is developed in order to describe the scattered EM field from the targets inside the building. If the building layout and imaging geometry is known, this problem can be exactly solved by using Maxwell's equations. However, the full wave solution is nonlinear in the targets $\left[\mathrm{LBM}^{+} 93\right]$. For example, the scattered field of two interacting targets is different from the superposition of the two individual single target solutions. This renders the inverse problem, i.e., inferring the position of the scatterers from the measurements also nonlinear and, thus, complex to solve $\left[\mathrm{LBM}^{+} 93\right]$. Hence, linear approximations of the forward scattering models are sought.

Common linear approximations include the Born approximation, Kirchhoff approximation, and Geometrical Optics (GO). The Born approximation assumes weak scatterers, such that the field inside targets is equivalent to the incident field [SS07]. The opposite, i.e., perfectly conducting targets, is assumed in the Kirchhoff (also called Physical Optics) approximation. Hence, only the surface of the target interacts with the field [SSPP09]. The GO or ray tracing approach uses a local plane wave assumption or "rays of light" to model the propagation of the wave [AA08b]. The latter is the simplest propagation model and is most commonly used in TWRI. As it is very intuitive and computationally cheap, it is used throughout this thesis.

The chapter starts with the basic signal models for Ultra-Wideband (UWB) radar in Section 3.1 and stepped-frequency radar in Section 3.2. An elaborate discussion of indirect or multipath propagation follows in Section 3.3. Various propagation modes are discussed and a multipath signal model for TWRI is developed. Subsequently, Section 3.4 deals with the reflections from building walls and corners, which are very strong scatterers in TWRI. Further, Section 3.5 discusses subsampling schemes for UWB and stepped-frequency radar that lend themselves for efficient data acquisition in a radar imaging context.

The material presented in this chapter is partly taken from [LAAZ14b, LZ14, LAAZ15a]. 


\subsection{Ultra-Wideband Signal Model}

First, the signal model for an UWB radar system with $M$ transmitters and $N$ receivers is introduced. The radar system operates sequentially, such that only one transmitter is active at a time while all receivers are recording. Such a real aperture system is generally of bistatic or multistatic nature as the transmitter and receiver locations are not the same. The model also includes a synthetic aperture system with a single transceiver by setting $M=N$ and considering only transmission and reception from a single location at a time. This translates to a monostatic array with $M=N$ elements. Utilizing UWB pulses is desirable for imaging scenes with stationary and moving target. If only stationary targets are present, a stepped-frequency radar may be used, which is discussed later [AA08a].

The signal model assumes that targets follow a linear motion with constant velocity in a 2D space, where stationary targets simply have zero velocity. Each transmitter sends $K$ wideband pulses with a Pulse Repetition Interval (PRI) of $T_{\mathrm{r}}$ within the Coherent Processing Interval (CPI). The pulse index $k=0, \ldots, K-1$, is referred to as slow time. The indoor targets are assumed to be moving slowly. This ensures that the target are approximately stationary during the interval $M T_{\mathrm{r}}$ where the transmitters are transmitting sequentially. Furthermore, the targets do not move out of a resolution cell during the observation interval $K M T_{\mathrm{r}}$. Note that the multiplexing time of the transmitters and the PRI could also be chosen independently to achieve the two above mentioned assumptions. The position of the $p$-th target at pulse $k$ is described as

$$
\boldsymbol{x}_{p}(k)=\left(x_{p}+v_{x p} k T_{\mathrm{r}}, y_{p}+v_{y p} k T_{\mathrm{r}}\right), \quad k=0, \ldots, K-1,
$$

in a Cartesian coordinate system, where $\left(x_{p}, y_{p}\right)$ are the initial positions and $\left(v_{x p}, v_{y p}\right)$ are the respective velocities for $p=0, \ldots, P-1$.

The transmitters and receivers can be placed outside of the building in an arbitrary configuration. For practical reasons, uniform linear arrays are usually used where the array elements are placed on a line with uniform element spacing. The transmitters emit a modulated wideband pulse with duration $T_{p}$ that can be described as $\Re\left\{s(t) \exp \left(j 2 \pi f_{c} t\right)\right\}$, where $t$ is the so-called fast time, $s(t)$ is the pulse in the complex baseband and $f_{c}$ is the carrier frequency. An illustration of the geometry of the scene and radar system are depicted in Figure 3.1. When the $m$-th transmitter is active, the received signal in the complex baseband corresponding to the $n$-th receiver, $k$-th pulse and $p$-th target can be expressed as a shifted and attenuated pulse

$$
\begin{aligned}
z_{m n k}^{p}(t)= & \sigma_{p} s\left(t-k M T_{\mathrm{r}}-m T_{\mathrm{r}}-\tau_{p m n}(k)\right) \\
& \times \exp \left(-j 2 \pi f_{c}\left(k M T_{\mathrm{r}}+m T_{\mathrm{r}}+\tau_{p m n}(k)\right)\right),
\end{aligned}
$$




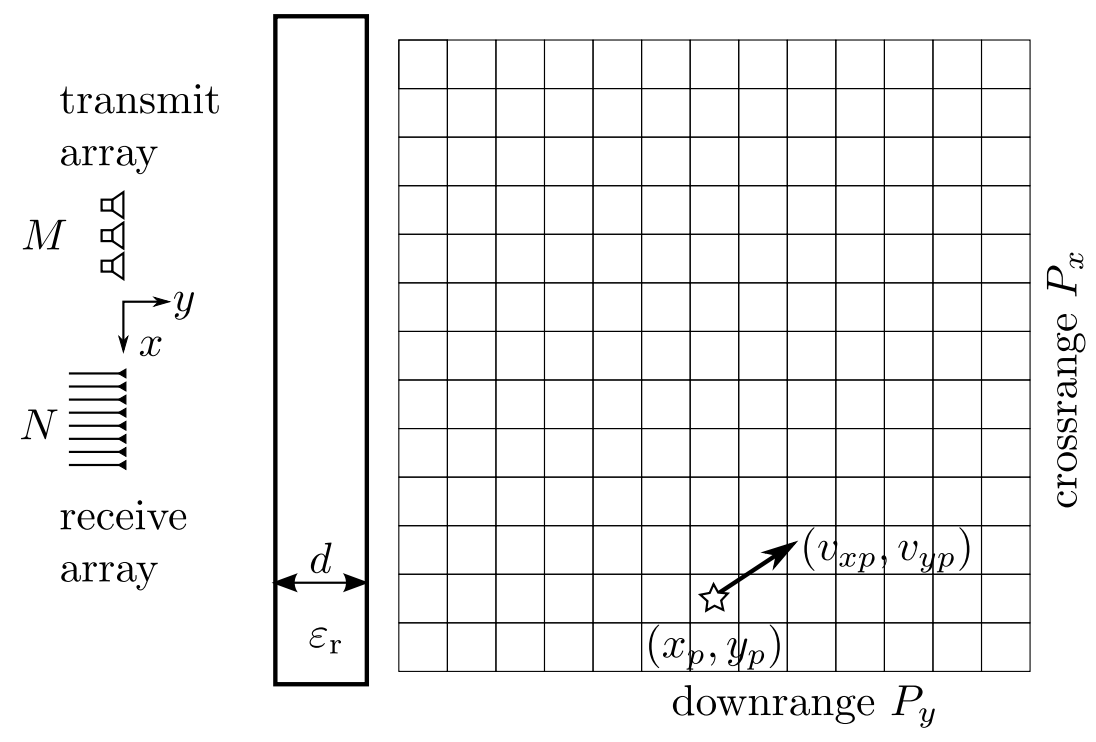

Figure 3.1. Geometry of the imaging system.

where $\tau_{p m n}(k)$ is the bistatic two-way delay, $\sigma_{p}$ is the reflectivity of the $p$-th point target, and $m=0, \ldots, M-1, n=0, \ldots, N-1$. The target reflectivity or Radar Cross Section (RCS) depends heavily on the nature of the target. For complex targets, it depends on the aspect and bistatic angles, as well as the frequency [RSH10]. The aspect angle describes the orientation of the target whereas the bistatic angle is the angle between the impinging and the reflected wave. Throughout this thesis, a deterministic and frequency independent target reflectivity is assumed. Further, the assumption of small arrays leads to little variations in the angles and, hence, a constant RCS for all transmitter/receiver pairs. The propagation losses are absorbed into the target reflectivities. Note that the propagation delays only depend on the locations of transmitter, target, and receiver, as well as the slow time index $k$. Each receiver collects the superposition of all target responses corresponding to the $m$-th transmitter and the $k$-th pulse. This can be written by

$$
\begin{aligned}
z_{m n k}(t)=\sum_{p=0}^{P-1} & \sigma_{p}\left(t-k M T_{\mathrm{r}}-m T_{\mathrm{r}}-\tau_{p m n}(k)\right) \\
& \quad \times \exp \left(-j 2 \pi f_{c}\left(k M T_{\mathrm{r}}+m T_{\mathrm{r}}+\tau_{p m n}(k)\right)\right) .
\end{aligned}
$$

For now, only the direct returns, i.e., the wave traveling on the shortest possible path, to and from the targets are considered. Note that the wave does not travel on a straight line as it is subject to refraction in the front wall. In fact, it is refracted at the outer and inner interfaces of the front wall on the way from the transmitter to the target as well as on the way back to the receiver. Indirect or multipath propagation as well as the calculation of the propagation delays is discussed in Section 3.3; wall returns are treated in Section 3.4. 
The model in (3.3) is discretized in time, velocity, and space and vectorized to obtain a discrete linear model of the system. The target space is discretized into a grid with size $P_{x} \times P_{y}$ and similarly, the velocities are sampled on a discrete grid with size $P_{v_{x}} \times P_{v_{y}}=P_{v}$, see Figure 3.1. In this $4 \mathrm{D}$ space, targets with any possible location or velocity are described by their reflectivities, whereas a non-existing target is represented by a zero reflectivity. In total there are $P_{x} P_{y} P_{v_{x}} P_{v_{y}}=P$ possible target states, that are stacked into a $P \times 1$ vector $\boldsymbol{\sigma}$. Also, the received signals $z_{m n k}(t)$ are uniformly sampled in at $T$ time steps with sampling interval $T_{s}$. The sampling interval is chosen such that the Nyquist rate of the transmit pulse $s(t)$ is attained.

After stacking the samples, a received signal vector $\boldsymbol{z}_{m n k}$ is obtained for each transmitter, receiver and pulse

$$
\boldsymbol{z}_{m n k}=\boldsymbol{\Psi}_{m n k} \boldsymbol{\sigma}, \quad m=0, \ldots, M-1, n=0, \ldots, N-1, k=0, \ldots, K-1
$$

where $\boldsymbol{\Psi}_{m n k} \in \mathbb{R}^{T \times P}$ are the dictionary matrices which are obtained by discretizing the right hand side of (3.2) and are defined as

$$
\begin{gathered}
{\left[\boldsymbol{\Psi}_{m n k}\right]_{i, p}=\left(t_{i}-k M T_{\mathrm{r}}-m T_{\mathrm{r}}-\tau_{p m n}(k)\right) \cdot \exp \left(-j 2 \pi f_{c}\left(k M T_{\mathrm{r}}+m T_{\mathrm{r}}+\tau_{p m n}(k)\right)\right),} \\
i=0, \ldots, T-1, \quad p=0, \ldots, P-1 .
\end{gathered}
$$

Stacking (3.4) for $M$ transmitters, $N$ receivers and $K$ pulses results in a $T M N K \times 1$ measurement vector $\boldsymbol{z}$ and a $T M N K \times P$ dictionary matrix $\boldsymbol{\Psi}$, yields

$$
z=\Psi \sigma
$$

with

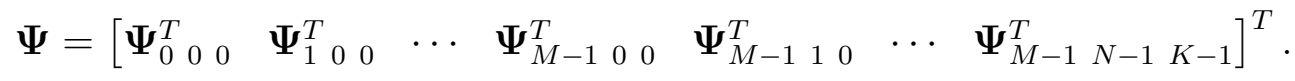

This linear measurement model can be exploited for the CS formulation of the scene reconstruction.

\section{$3.2 \quad$ Stepped-Frequency Signal Model}

In this section, the special case of purely stationary targets is considered. The stationary target model can be equivalently expressed in the frequency domain formulation. The frequency domain model is more appropriate to describe measurements from a stepped-frequency radar. 
The time domain model (3.3) is transformed to the Frequency Domain (FD), assuming a single pulse

$$
z_{\mathrm{FD}, m, n}(f)=\sum_{p=0}^{P_{x} P_{y}-1} S(f) \sigma_{p} \exp \left(-j 2 \pi\left(f+f_{c}\right) \tau_{p m n}\right)
$$

where $f$ is the continuous frequency variable and $S(f)$ is the Fourier transform of the wideband transmit pulse $s(t)$. The FD signal is discretized into $L$ frequencies, such that $\left\{f_{l}\right\}_{l=0}^{L-1}$ cover the desired frequency band. Further, regularly spaced frequency steps around the center frequency $f_{c}$ are assumed. The FD model (3.8) is now discretized as

$$
z_{\mathrm{FD}}[l, m, n]=\sum_{p=0}^{P_{x} P_{y}-1} S\left(f_{l}\right) \sigma_{p} \exp \left(-j 2 \pi f_{l} \tau_{p m n}\right) .
$$

This so-called stepped-frequency model is commonly used in TWRI for example in [AA08a, LAAZ14b].

Using the same stacking principle as delineated above, (3.9) can be expressed in vector matrix formulation

$$
\boldsymbol{z}_{\mathrm{FD}}=\boldsymbol{\Psi}_{\mathrm{FD}} \boldsymbol{\sigma}
$$

where $\boldsymbol{z}_{\mathrm{FD}}=\left[z_{\mathrm{FD}}[0,0,0], z_{\mathrm{FD}}[1,0,0], \ldots, z_{\mathrm{FD}}[L-1, M-1, N-1]^{T}\right.$. The FD dictionary $\boldsymbol{\Psi}_{\mathrm{FD}} \in \mathbb{R}^{L M N \times P_{x} P_{y}}$ contains weighted versions of the phase terms.

Alternatively, stationary scenes are included in the UWB model (3.3) as targets with zero velocity. When considering stationary scenes, the target state vector $\boldsymbol{\sigma}$ can be simplified to cover the spatial domain only. Also, the propagation delays do not change from pulse to pulse, hence, a single pulse is sufficient to interrogate the scene. Note that reducing the number of pulses leads to a degradation of the Signal-to-Noise Ratio (SNR) of the measured data. The time domain model (3.6) and the frequency domain model (3.10) have the same structure. Hence, they can be used interchangeably as long as the targets are not moving. In this thesis, the FD model is used for stationary targets and the time domain model is used if moving targets are involved.

\subsection{Multipath Propagation}

In this section, the various cases of multipath propagation are categorized and discussed. This is later used to extend the direct path received signal model to account 
for the contribution of indirect propagation. Only propagation paths that actually interact with the targets of interest are discussed here. Propagation modes that interact solely with the walls are treated in Section 3.4.

In contrast to the direct path, multipath propagation corresponds to indirect paths. These involve reflections at one or more secondary reflectors other than the target of interest. In TWRI the secondary scatterer could be for example an interior wall, the floor/ceiling or another target in the room. Depending on the characteristic reflections, multipath can be divided into the following categories [LAAZ14b].

- Wall Ringing Multipath: Multiple reflections inside the front wall when the wave travels to/from the target.

- Interior Wall Multipath: Specular reflection at one or more interior walls.

- Floor/Ceiling Multipath: Specular reflections at the floor and/or ceiling.

- Target Interaction Multipath: The wave traveling along this path interacts with more than one target.

In this thesis, only interior wall multipath and wall ringing multipath are considered. Floor/ceiling multipath are neglected as they are usually not present when using antennas with a narrow vertical beamwidth. If present, floor/ceiling multipath can be treated in the same manner as interior wall multipath as it corresponds to specular reflection at a large smooth surface. Target interaction multipath results in a non-linear signal model as the targets cannot be treated independently anymore. However, if the targets are sufficiently separated, diffuse scattering leads to large attenuation for this type of multipath. Hence, target interaction multipath is not considered.

Interior wall multipath involves specular reflection as the surface roughness of a building wall is usually much smaller than the wavelengths in TWRI. It is further subdivided into the following classes:

- First order multipath: This scattering scenario involves a direct propagation to the target on transmit and one secondary reflection at an interior wall on the way back to the receiver, or vice versa. Hence, the path to the target is different from the path back to the receiver. This is the dominant case of multipath in TWRI. Note that even for a monostatic radar, the scattering at the target is of bistatic nature. 
- Second order multipath: The signal on the round-trip path is reflected twice at an interior wall. Two cases can be further distinguished:

- Quasi-monostatic: There are two specular reflections on the round-trip path, each of which occurs on the same interior wall. This corresponds to monostatic scattering at the target for a monostatic radar. When using a bistatic radar with a small baseline the bistatic angle is very small compared to first order multipath.

- Bistatic: The two specular reflections take place at two different walls.

- Higher-order multipath: Three or more specular reflections occur during the round-trip path.

Throughout this thesis, first order multipath and quasi-monostatic second order multipath from interior walls are considered. As the signal is attenuated at each secondary reflection, second and higher-order multipath returns are usually weak enough to be safely neglected. However, (quasi-)monostatic second order multipath is included in the model for the following reason: Most targets show stronger reflectivity for small bistatic angles [RSH10]. This may result in a significant contribution of this propagation type. For now, perfect knowledge of the building layout, namely, location, thickness, and permittivity of the front wall, as well as the location of the interior walls, is assumed. Inaccurate or unknown model parameters are treated in Section 5.3. Thus, multipath can be approximated by the GO model using a finite number of discrete paths, which is later used in the CS reconstruction method. In the following subsections, the calculation of the propagation delays is described as well as the assembly of the full multipath model.

\subsubsection{Direct Path and Wall Ringing Multipath}

When an EM wave hits the planar interface between medium $\mathrm{A}$ and medium $\mathrm{B}$, it is partly refracted into medium B and partly reflected back to medium A [Bal89]. However, part of the signal on transit to/from the target may undergo multiple reflections inside the front wall [KKT08]. Two cases of this effect are distinguished, namely wall ringing and wall reverberation. Wall reverberation refers to the part of the wave that interacts solely with the front wall and never reaches the targets inside the room. This results in multiple wall responses in the received signal and will be analyzed in Section 3.4. Wall ringing describes the portion of the signal that reaches the targets of interest. In contrast to the direct path, the signal undergoes multiple reflections within 


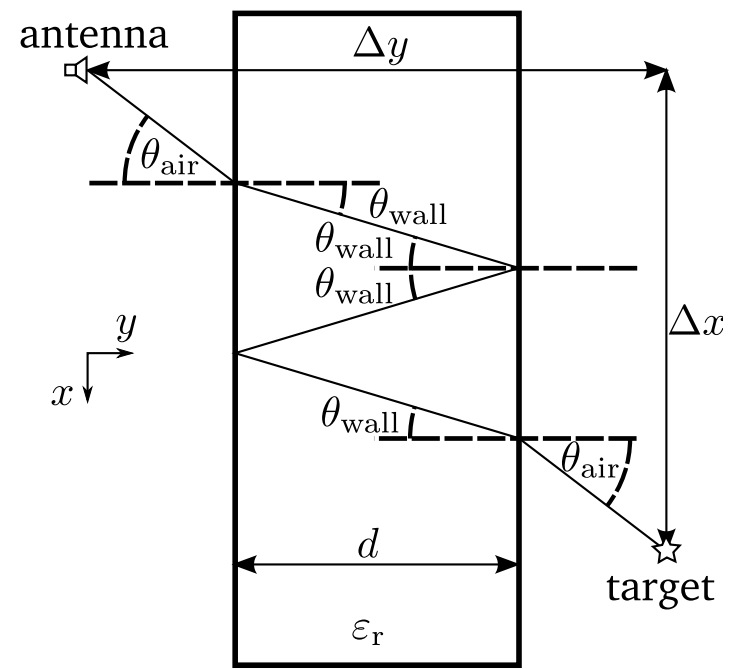

Figure 3.2. Wall ringing propagation with $b=1$ internal bounces.

the front wall before reaching the target and/or receiver. In the radar image this results in multiple decaying copies of the target equally spaced in the range direction.

Figure 3.2 depicts the front wall along with the incident, reflected, and refracted waves associated with a certain target/receiver pair. A front wall with thickness $d_{\text {wall }}$ and relative permittivity $\varepsilon_{r}$ is assumed. The distance between the target and the receiver in crossrange direction, $\Delta x$, can be expressed as

$$
\Delta x=(\Delta y-d) \tan \theta_{\text {air }}+d(1+2 b) \tan \theta_{\text {wall }},
$$

where $\Delta y$ is the distance between target and array element in downrange direction, and $\theta_{\text {air }}$ and $\theta_{\text {wall }}$ are the angles in the air and in the wall medium, respectively. The integer $b$ denotes the number of internal reflections within the wall. The case $b=0$ describes the direct path as derived in [AA08a]. The relationship between the angles of incidence and refraction are governed by Snell's law,

$$
\frac{\sin \theta_{\text {air }}}{\sin \theta_{\text {wall }}}=\sqrt{\varepsilon_{\mathrm{r}}} .
$$

Equations (3.11) and (3.12) form a nonlinear system of equations that can be solved numerically for the unknown angles, e.g., using Newton's method. Having obtained the angles $\theta_{\text {air }}$ and $\theta_{\text {wall }}$, the one-way propagation delays can be calculated as [AA08a]

$$
\tau(\Delta x, \Delta y, b)=\frac{(\Delta y-d)}{c \cos \theta_{\text {air }}}+\frac{\sqrt{\varepsilon_{\mathrm{r}}} d(1+2 b)}{c \cos \theta_{\text {wall }}},
$$

where $c$ is the propagation speed in vacuum. As the direction of propagation is not relevant, the one-way delay from the transmitter to the target is obtained in exactly the 


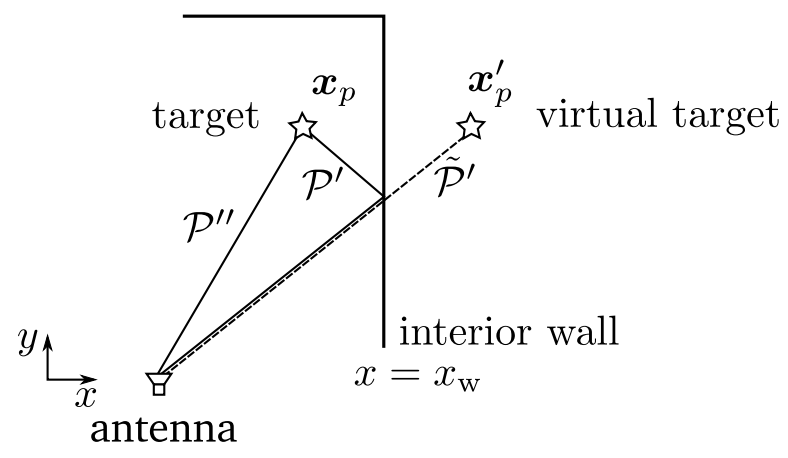

Figure 3.3. Multipath propagation via reflection at an internal wall.

same way. A frequency independent wall medium is assumed. For real wall media, such as concrete, the permittivity may depend on the frequency which leads to a distortion of the wideband pulse.

\subsubsection{Interior Wall Multipath}

As stated above, the second type of multipath considered in this work is interior wall multipath. Since this type arises from specular reflections at a large smooth surface, a virtual target is used to describe the effect. Refer to the illustration in Figure 3.3, where the front wall has been omitted for the sake of simplicity. The scene consists of a target located at $\boldsymbol{x}_{p}=\left[x_{p}, y_{p}\right]^{T}$ and one interior wall (side wall of the room) parallel to the $y$-axis and located at $x=x_{\mathrm{w}}$. Multipath propagation may occur along the path $\mathcal{P}^{\prime}$ from the target via secondary reflection at the interior wall back to a receiver. The multipath can be expressed as a direct return path $\tilde{\mathcal{P}}^{\prime}$ from a virtual target located at $\boldsymbol{x}_{p}^{\prime}=\left[2 x_{\mathrm{w}}-x_{p}, y_{p}\right]^{T}$. Due to the laws of reflection [Bal89] (angle of incidence equals angle of reflection), the location of the virtual target is found by reflecting the original target at the wall. From the receiver perspective, the two paths are equivalent in terms of delay and angle of incidence. Hence, the problem of calculating a oneway propagation delay for the interior wall multipath can be traced back to the direct propagation case. Note that the case of the transmit path and reflection from a different wall can be treated likewise.

For the pair of receiver $n$ and target $p$, the delay is denoted by $\tau_{p n}^{\left(\mathcal{P}^{\prime}\right)}$, which is equivalent to $\tau_{p n}^{\left(\tilde{\mathcal{P}}^{\prime}\right)}$. Without the front wall, the delay can simply be calculated as the Euclidean distance divided by the propagation speed. If the front wall is present, the double refraction at the two wall interfaces have to be considered. As the two paths $\mathcal{P}^{\prime}$ and $\tilde{\mathcal{P}}^{\prime}$ are equivalent, the calculation can be carried out in the same fashion as described in the previous section. 


\subsubsection{Bistatic Received Signal Model}

All possible and significant propagation paths occur simultaneously. Utilizing the described multipath mechanisms, a comprehensive bistatic received signal model is derived. A round-trip path $\mathcal{P}$ consists of two one-way paths, namely the path $\mathcal{P}^{\prime \prime}$ from the transmitter to the scattering target and the path $\mathcal{P}^{\prime}$ from the target back to the receiver. The one-way path $\mathcal{P}^{\prime}$ is the direct path or any type of multipath as described above. Hence, there exist $R_{1}$ return paths from a certain target back to the receiver, which are denoted as $\mathcal{P}_{r_{1}}^{\prime}, r_{1}=0, \ldots, R_{1}-1$. The same observation holds for the one-way transmit paths, which are denoted by $\mathcal{P}_{r_{2}}^{\prime \prime}, r_{2}=0, \ldots, R_{2}-1$. Hence, for the round-trip path $\mathcal{P}_{r}, r=0, \ldots, R-1$ one can conclude a maximum number of $R \leq R_{1} R_{2}$ paths, which represent all possible combinations of one-way paths. A function can be established describing these combinations by mapping the index $r$ of the round-trip path to a pair of indices of the one-way paths, $r \mapsto\left(r_{1}, r_{2}\right)$. Note that $R_{1} R_{2}$ is the maximum possible number of round-trip paths. However, some paths $\mathcal{P}_{r}$ belong to the class of higher-order multipath which is strongly attenuated and, thus, can be ignored. In the remainder of this work, $\mathcal{P}_{0}$ denotes the direct path, i.e., the case without any multipath. Note that the paths relate to propagation modes or generic path types as they are independent of the exact location of transmitter, receiver or target. Consider the illustrations in Figures 3.4 and 3.5 to clarify the above described model in a bistatic radar configuration. Figure 3.4 depicts three possible return paths. The direct propagation path is marked as $\mathcal{P}_{0}^{\prime}$, secondary reflection from a side wall is denoted as $\mathcal{P}_{1}^{\prime}$, and $\mathcal{P}_{2}^{\prime}$ indicates wall ringing. Three equivalent transmit paths are present for the propagation from the transmitter to the scatterer. The combination of three transmit paths and three return paths results in a total of nine round-trip paths, as shown in Figure 3.5. The paths $\mathcal{P}_{1}, \mathcal{P}_{2}, \mathcal{P}_{3}$ and $\mathcal{P}_{6}$ correspond to first order multipath, $\mathcal{P}_{4}$ is a quasi-monostatic second order multipath, which is also included in the model. Round-trip paths $\mathcal{P}_{5}$ and $\mathcal{P}_{7}$ are general second order multipaths that are ignored due to higher attenuation. A round-trip with two wall ringing paths, as $\mathcal{P}_{8}$ may or may not be included, depending on the attenuation properties of the front wall. In the case of a monostatic configuration, the number of different round-trip paths decreases due to symmetry. For example $\mathcal{P}_{1}$ and $\mathcal{P}_{3}$ or $\mathcal{P}_{5}$ and $\mathcal{P}_{7}$ would be equivalent.

From the topological description of multipath follows the calculation of the round-trip propagation. A round-trip path $\mathcal{P}_{r}$ consists of the one-way paths $\mathcal{P}_{r}^{\prime}$ and $\mathcal{P}_{r}^{\prime \prime}$. Thus, for each combination of path type, target, transmitter and receiver, the associated delay is calculated as

$$
\tau_{p m n}^{\left(\mathcal{P}_{r}\right)}=\tau_{p m n}^{\left(\mathcal{P}_{r_{1}}^{\prime}\right)}+\tau_{p m n}^{\left(\mathcal{P}_{r_{2}}^{\prime \prime}\right)}
$$

The propagation delays for the one-way paths can be found by using the principles 


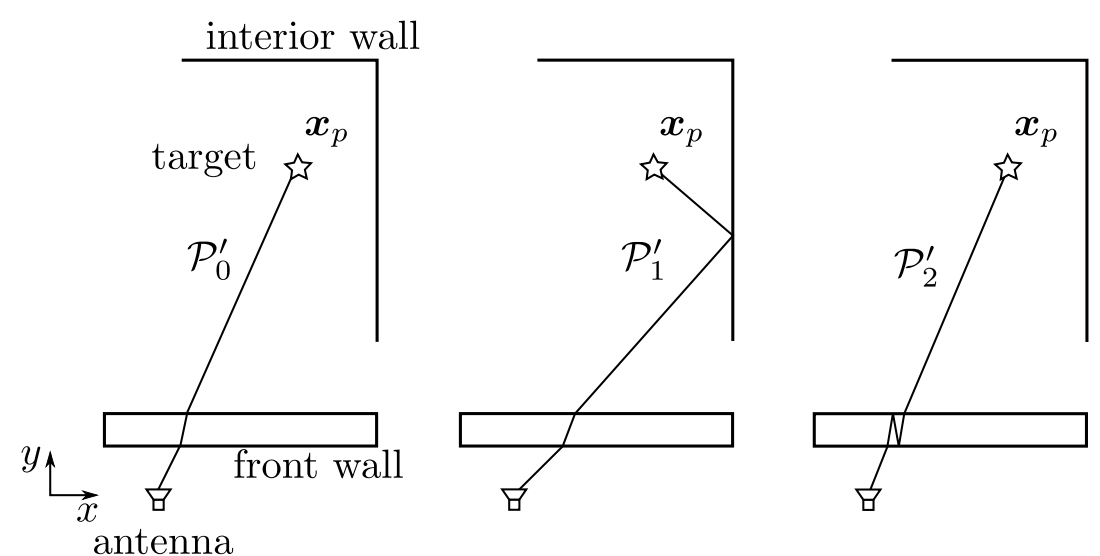

Figure 3.4. Example for three possible one-way return paths. The paths $\mathcal{P}_{0}^{\prime}, \mathcal{P}_{1}^{\prime}$ and $\mathcal{P}_{2}^{\prime}$ correspond to direct propagation, secondary reflection at a side wall and wall ringing, respectively.

delineated above. For notational convenience, the round-trip delay between the $m$-th transmitter, $p$-th target and $n$-th receiver associated with path $\mathcal{P}_{r}$ is denoted as $\tau_{p m n}^{(r)}$. The slow time dependency of the delays can be treated in the same fashion. It has been omitted for simplicity.

In a similar manner, the complex amplitudes $\Gamma_{p m n}^{\left(\mathcal{P}_{r}\right)} \in \mathbb{C}$ associated with each possible combination of path, transmitter, receiver, and target can be calculated. With each reflection and refraction, the traveling wave suffers from attenuation and, possibly, a phase shift. For each one-way path, the complex amplitude $\Gamma_{p m n}^{(\cdot)}$ can be derived from the dielectric properties of the front and sidewalls and the corresponding angles of incidence and refraction. For a detailed derivation refer to Appendix A.1.

The incident, refraction, and reflection angles, associated with one path do not vary much across the array. Thus, the complex amplitudes can be simplified as

$$
\Gamma_{p m n}^{\left(\mathcal{P}_{r}\right)} \approx \Gamma_{p}^{\left(\mathcal{P}_{r}\right)}, \quad p=0, \ldots, P-1, \quad m=0, \ldots, M-1, \quad n=0, \ldots, N-1 .
$$

In other words, the complex amplitude for each path depends only on the target position. This approximation generally holds in far-field conditions where all angles are approximately equal across all target/array element pairs. However, it is assumed that the array baseline is sufficiently small as compared to the distance to the scene. Thus, the approximation also holds in the near-field case. Refer to Appendix A.2 for an estimation of the conditions under which this assumption holds.

The multipath propagation delays and amplitudes are used to establish a received signal model for multipath environments. The phase and amplitude of the reflectivity of a 

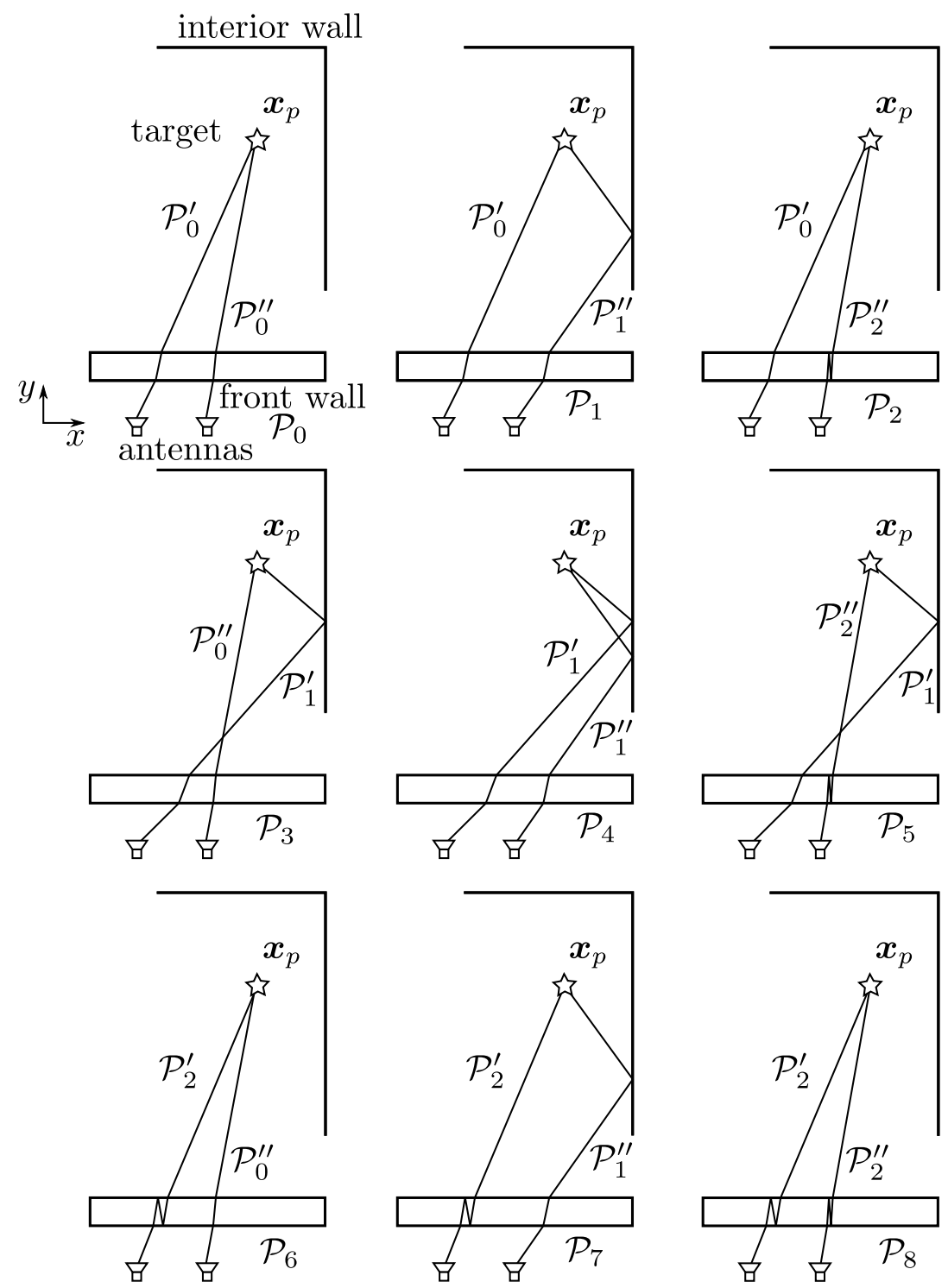

Figure 3.5. Round-trip paths between transceiver and target for the partial paths shown in Figure 3.4. 
target changes in general with the bistatic angle and aspect angle. Thus, an individual target reflectivity is assumed for each path. Observe that the complex amplitude only depends on the target and path indices. Hence, they can be absorbed into the pathdependent target reflectivity vectors for notational convenience. Each receiver picks up a superposition of all possible propagation paths $r=0, \ldots, R-1$. Hence, (3.6) is extended to

$$
\boldsymbol{z}=\boldsymbol{\Psi}^{(0)} \boldsymbol{\sigma}^{(0)}+\boldsymbol{\Psi}^{(1)} \boldsymbol{\sigma}^{(1)}+\cdots+\boldsymbol{\Psi}^{(R-1)} \boldsymbol{\sigma}^{(R-1)},
$$

where $\boldsymbol{\sigma}^{(r)}$ are the individual target reflectivity vectors for each path. The dictionaries $\Psi^{(r)}$ are defined according to (3.5) and (3.7), where $\tau_{p m n}(k)$ is replaced by $\tau_{p m n}^{(r)}(k)$. Equivalently, the measurement model can be expressed for stationary scenes using the stepped-frequency approach

$$
\boldsymbol{z}_{\mathrm{FD}}=\boldsymbol{\Psi}_{\mathrm{FD}}^{(0)} \boldsymbol{\sigma}^{(0)}+\boldsymbol{\Psi}_{\mathrm{FD}}^{(1)} \boldsymbol{\sigma}^{(1)}+\cdots+\boldsymbol{\Psi}_{\mathrm{FD}}^{(R-1)} \boldsymbol{\sigma}^{(R-1)} .
$$

Equations (3.16) and (3.17) are generalizations of the non-multipath propagation models (3.3) and (3.8), respectively. If the number of propagation paths is set to $R=1$, the multipath signal models are equivalent to the direct path models. Using these linear measurement models, CS can be applied to achieve an accurate reconstruction of the scene. In practice, the number of multipath contributions is limited by the number of large flat surfaces. Thus, for monostatic radar imaging of a single room, one would expect $R=4$ propagation paths: one direct path and three propagation paths due to the interior walls as multipath. This number increases if second-order multipaths, wall ringing or a bistatic operation are considered.

\subsection{Direct Wall Reflections}

So far target returns have been considered. That is propagation modes interacting with the targets of interest at some stage. In TWRI, however, a significant part of the wave never reaches the scene of interest before returning to the receiver. The part of the signal corresponding to direct wall returns is modeled and analyzed in this section.

Several of these direct wall reflections can be distinguished:

- Front Wall Reflection: Direct reflection from the outer surface of the front fall.

- Interior Wall Reflection: Direct reflections from one or more interior walls parallel to the front wall. 
- Wall Reverberation: Multiple reflections within the front wall that do not enter the scene of interest.

- Corner Reflections: Reflections from the corners formed by any two perpendicular interior walls.

The first three cases can be expressed by the same model. The returns originate from specular reflection at large smooth surfaces. For small arrays, the walls can be regarded as infinitely long slabs. Hence, there is a shift invariance along the crossrange coordinate. The wall response corresponding to transmitter $m$, receiver $n$, and pulse $k$ can be expressed as

$$
\begin{aligned}
z_{m n k}^{\text {wall }}(t)=\sum_{q=0}^{Q-1} & \sigma_{q}^{\text {wall }}\left(t-k M T_{\mathrm{r}}-m T_{\mathrm{r}}-\tau_{q m n}^{\text {wall }}\right) \\
& \quad \times \exp \left(-j 2 \pi f_{c}\left(k M T_{\mathrm{r}}+m T_{\mathrm{r}}+\tau_{q m n}^{\text {wall }}\right)\right) .
\end{aligned}
$$

The complex reflectivity of the $q$-th wall component is denoted as $\sigma_{q}^{\text {wall }}$. Furthermore, $\tau_{q m n}^{\text {wall }}$ is the two-way delay from the transmitter to the wall component and back to the receiver. The calculation of the wall delays can be carried out in the same fashion as the target delays. However, the specular reflection condition at the reflecting wall surface has to be considered. If transmitter and receiver have the same distance to the front wall, the delay of interest is equivalent to the delay of a target on the wall surface in the midpoint between transmitter and receiver along the crossrange coordinate. In the monostatic case, this means that the equivalent reflection point is at the same crossrange as the transceiver. Thus, the delay is independent of the crossrange position of the transceiver. The delays associated with the wall returns do not vary with the slow time index $k$ as the wall is a stationary reflector. The decrease in amplitude for the reverberation paths is accounted for in the wall reflectivities $\sigma_{q}^{\text {wall }}$. In a monostatic setup, up to three wall reverberation responses are typically observed [THAD11]. Due to the strong attenuation in wall materials, higher order reverberations drop below the noise floor. In bistatic configurations, oblique incidence leads to even strong attenuation of wall reverberations. Herein, the frequency dependent attenuation behavior of wall materials is not considered.

Perpendicular building walls form corners that can be regarded as dihedral reflectors. These corners can be modeled as stationary targets. However, their reflectivity depends on the angles of incidence and reflection. The returns from $N_{\mathrm{c}}$ corners can be expressed as

$$
\begin{aligned}
z_{m n k}^{\text {corner }}(t) & =\sum_{u=0}^{N_{\mathrm{c}}-1} \sigma_{u m n}^{\text {corner }} s\left(t-k M T_{\mathrm{r}}-m T_{\mathrm{r}}-\tau_{u m n}^{\text {corner }}\right) \\
& \times \exp \left(-j 2 \pi f_{c}\left(k M T_{\mathrm{r}}+m T_{\mathrm{r}}+\tau_{u m n}^{\text {corner }}\right)\right)
\end{aligned}
$$


where $\sigma_{u m n}^{\text {corner }}$ is the reflectivity of the $u$-th corner and $\tau_{u m n}^{\text {corner }}$ is the two-way propagation delay between the $m$-th transmitter, the $u$-th corner and the $n$-th receiver. Note that, similar to the wall returns, the delay is independent of the slow time index $k$. However, the corner reflectivity depends on the transmitter and the receiver locations. The delay can be calculated in the same way as for point targets, while the reflectivity is given by [JRM10]

$$
\begin{aligned}
\sigma_{u m n}^{\text {corner }} & =\left(\frac{j 2 \sqrt{\pi} f_{c}}{c}\right)^{1 / 2} 2 L_{u} \times \operatorname{sinc}\left[\frac{4 \pi L_{u} f_{c}}{c}\left(\cos \left(\psi_{u m}^{\mathrm{t}}-\tilde{\psi}_{u}\right)-\cos \left(\psi_{u n}^{\mathrm{r}}-\tilde{\psi}_{u}\right)\right)\right] \\
& \times \begin{cases}\sin \left(\frac{\psi_{u m}^{\mathrm{t}}+\psi_{u n}^{\mathrm{r}}-2 \tilde{\psi}_{u}}{2}\right), & \psi_{u n}^{\mathrm{r}}, \psi_{u m}^{\mathrm{t}} \in\left[\tilde{\psi}_{u}, \tilde{\psi}_{u}+\frac{\pi}{4}\right] \\
\cos \left(\frac{\psi_{u m}^{\mathrm{t}}+\psi_{u n}^{\mathrm{r}}-2 \tilde{\psi}_{u}}{2}\right), & \psi_{u n}^{\mathrm{r}}, \psi_{u m}^{\mathrm{t}} \in\left[\tilde{\psi}_{u}+\frac{\pi}{4}, \tilde{\psi}_{u}+\frac{\pi}{2}\right] .\end{cases}
\end{aligned}
$$

Hereby, $L_{u}$ is the length of the sides of the $u$-th corner, $\tilde{\psi}_{u}$ is the orientation angle of the $u$-th corner, $\psi_{u m}^{\mathrm{t}}, \psi_{u n}^{\mathrm{r}}$ are the respective angles of incidence and reflection, and $c$ is the speed of light. The angles are measured counterclockwise from the positive $x$-axis.

The target returns (3.3), wall returns (3.18), and corner returns (3.19) are received simultaneously. This results in a total received signal for $m=0, \ldots, M-1, n=$ $0, \ldots, N-1, k=0, \ldots, K-1$

$$
z_{m n k}^{\mathrm{tot}}(t)=z_{m n k}(t)+z_{m n k}^{\mathrm{wall}}(t)+z_{m n k}^{\mathrm{corner}}(t)+n(t),
$$

where $n(t)$ is independent and identically distributed (i.i.d.) additive white sensor noise. The discretization and stacking of this model can be carried out in the same fashion as in the above target model. Throughout this thesis, the propagation model (3.21) is used. Depending on the focus of the respective chapter, a subset of target, wall and corner returns is selected.

\subsection{Efficient Sampling Schemes}

The essential idea of CS is to achieve good reconstruction results with less data. In this section, various efficient sampling schemes are discussed which are tailored to specific radar systems.

Instead of taking measurements at full Nyquist rate, a sampling scheme with lower data rate is devised. Various measurement schemes have been proposed in [YA08, AAZ11, GMS09b, GMS09a, ZAA ${ }^{+}$12] to reduce the volume of the acquired data. All schemes can be characterized using a downsampling matrix $\Phi \in \mathbb{R}^{J \times T M N K}$ acting on the full set of measurements. $J \ll T M N K$ is the number of reduced measurements. 
The compressively sampled version of the radar return in (3.16) can be expressed as

$$
\overline{\boldsymbol{z}}=\boldsymbol{\Phi} \boldsymbol{z}=\boldsymbol{\Phi}\left(\boldsymbol{\Psi}^{(0)} \boldsymbol{\sigma}^{(0)}+\boldsymbol{\Psi}^{(1)} \boldsymbol{\sigma}^{(1)}+\cdots+\boldsymbol{\Psi}^{(R-1)} \boldsymbol{\sigma}^{(R-1)}\right)+\boldsymbol{n},
$$

where $\boldsymbol{\Phi}$ represents the undersampling operation and $\boldsymbol{n}$ is an i.i.d. additive noise vector. Equivalently, in the stepped-frequency radar an undersampled measurement vector is obtained from model (3.17) as

$$
\overline{\boldsymbol{z}}_{\mathrm{FD}}=\boldsymbol{\Phi}_{\mathrm{FD}} \boldsymbol{z}_{\mathrm{FD}}=\boldsymbol{\Phi}_{\mathrm{FD}}\left(\boldsymbol{\Psi}_{\mathrm{FD}}^{(0)} \boldsymbol{\sigma}^{(0)}+\boldsymbol{\Psi}_{\mathrm{FD}}^{(1)} \boldsymbol{\sigma}^{(1)}+\cdots+\boldsymbol{\Psi}_{\mathrm{FD}}^{(R-1)} \boldsymbol{\sigma}^{(R-1)}\right)+\boldsymbol{n}_{\mathrm{FD}},
$$

where $\boldsymbol{n}_{\mathrm{FD}}$ is i.i.d. additive noise. The undersampling operation in the frequency domain is represented by $\boldsymbol{\Phi}_{\mathrm{FD}}$.

It is noted that in practical implementation of a CS radar, a reduced number of measurements would be directly acquired. Downsampling of the full set of measurements is used as a compact description of the sampling process. Hence, the challenge is to design $\boldsymbol{\Phi}$, such that on the one hand maximum cost and complexity reduction of the radar system is achieved. On the other hand a good reconstruction performance is desired. UWB radar and stepped-frequency radar are treated separately, as different requirements for the downsampling operation arise.

\subsubsection{Ultra-Wideband Pulse Radar}

First, UWB pulse radar as described in Section 3.1 is considered. The benefits of CS are maximized when the radar return is undersampled in all four dimensions, namely, fast time, slow time and transmit/receive elements. For the latter two, most savings are achieved by random omission of some elements. This leads to sparse transmit and receive arrays. Random undersampling of slow time does not yield any savings in terms of time or cost, as long as the first and the last pulses are retained in the CPI. However, reducing the number of pulses within the CPI reduces the required power. This may be desirable in portable applications. Various methods are available to compressively sample in the fast time. Here, a random mixing scheme is adopted. Each receiver correlates the signal with a set of random sequences and only the corresponding correlation result is sampled. For a detailed discussion of this scheme, the reader is referred to [GMS09b, QAA13].

The number of samples is reduced along transmit elements to $M_{\mathrm{d}}$, along receive elements to $N_{\mathrm{d}}$, along slow time to $K_{\mathrm{d}}$, and along fast time to $T_{\mathrm{d}}$. This is achieved by a measurement matrix constructed as [QAA13]

$$
\begin{aligned}
\boldsymbol{\Phi}= & \left(\boldsymbol{\Phi}^{\text {transmit }} \otimes \boldsymbol{I}_{N_{\mathrm{d}} K_{\mathrm{d}} T_{\mathrm{d}}}\right) \cdot\left(\boldsymbol{\Phi}^{\text {receive }} \otimes \boldsymbol{I}_{M K_{\mathrm{d}} T_{\mathrm{d}}}\right) \cdot\left(\boldsymbol{\Phi}^{\text {slow }} \otimes \boldsymbol{I}_{M N T_{\mathrm{d}}}\right) \\
& \cdot \operatorname{diag}\left(\boldsymbol{\Phi}_{0}^{\text {fast }}, \ldots, \boldsymbol{\Phi}_{M N K-1}^{\text {fast }}\right),
\end{aligned}
$$


where $\otimes$ denotes Kronecker product and $\boldsymbol{I}_{a}$ is an identity matrix of dimension $a$. The total number of reduced measurements is given by $J=M_{\mathrm{d}} N_{\mathrm{d}} K_{\mathrm{d}} T_{\mathrm{d}} \ll T M N K$. Each of the matrices for downsampling in the dimension of transmit elements $\boldsymbol{\Phi}^{\text {transmit }} \in$ $\mathbb{R}^{M_{\mathrm{d}} \times M}$, receive elements $\boldsymbol{\Phi}^{\text {receive }} \in \mathbb{R}^{N_{\mathrm{d}} \times N}$ and slow time $\boldsymbol{\Phi}^{\text {slow }} \in \mathbb{R}^{K_{\mathrm{d}} \times K}$ consists of randomly chosen rows from an identity matrix. Random mixing in fast time is achieved by Gaussian random matrices $\boldsymbol{\Phi}_{i}^{\text {fast }} \in \mathbb{R}^{T_{\mathrm{d}} \times T}$ with entries drawn from a standard normal distribution. Other random matrices, e.g., drawn from a Bernoulli distribution, may also be considered to achieve a good trade-off between ease of implementation and performance, see [GMS09b]. In order to achieve the reduced complexity of the receiver and the data reduction, the downsampling operation has to be implemented in hardware. The considered scheme lends itself to a hardware implementation using microwave mixers and low-pass filters [QAA13].

\subsubsection{Stepped-Frequency Radar}

For stepped-frequency operation, as considered in Section 3.2, efficient sampling schemes take a different form. A binary measurement matrix $\boldsymbol{\Phi}_{\mathrm{FD}} \in\{0,1\}^{J \times L M N}$ is applied to the full set of measurements. It consists of randomly chosen rows of an identity matrix. In the frequency domain, random mixing is not required. Short-time pulses are naturally incoherent in the Fourier domain. Hence, taking measurements at a few frequencies is sufficient, refer to Section 2.1.

Structured and unstructured downsampling operations have been considered in literature [YA08]. In the unstructured case, random triplets of transmitter, receiver and frequency bins are sampled. Thus, the downsampling matrix $\boldsymbol{\Phi}_{\mathrm{FD}}$ consists of randomly chosen rows from an identity matrix. In this case, all elements of the transmit and receive array take part in the measurement process.

In the structured case, $M_{\mathrm{d}}$ transmitters, $N_{\mathrm{d}}$ receivers and $L_{\mathrm{d}}$ frequencies are selected beforehand. Similar to downsampling in the UWB model, measurements are taken at any possible combination of these subsets. This can be expressed as

$$
\boldsymbol{\Phi}_{\mathrm{FD}}=\left(\boldsymbol{\Phi}_{\mathrm{FD}}^{\mathrm{freq}} \otimes \boldsymbol{I}_{M_{\mathrm{d}} N_{\mathrm{d}}}\right) \cdot\left(\boldsymbol{\Phi}_{\mathrm{FD}}^{\text {transmit }} \otimes \boldsymbol{I}_{L N_{\mathrm{d}}}\right) \cdot\left(\boldsymbol{\Phi}_{\mathrm{FD}}^{\text {receive }} \otimes \boldsymbol{I}_{L M}\right)
$$

The total number of reduced measurements is given by $J=L_{\mathrm{d}} M_{\mathrm{d}} N_{\mathrm{d}} \ll L M N$. As before, the partial downsampling matrices $\boldsymbol{\Phi}^{\text {transmit }} \in \mathbb{R}^{M_{\mathrm{d}} \times M}$, $\boldsymbol{\Phi}^{\text {receive }} \in \mathbb{R}^{N_{\mathrm{d}} \times N}$ and $\Phi^{\text {freq }} \in \mathbb{R}^{L_{\mathrm{d}} \times L}$ consist of randomly chosen rows from an identity matrix. This structured scheme has the advantage that some array elements become redundant and, hence, can be omitted to save hardware costs. 



\section{Chapter 4}

\section{Sparsity-Based Multipath Exploitation}

This chapter focuses on sparse reconstruction of targets in an indoor environment. Due to the front wall and surrounding scatterers, multipath propagation arises which is exploited to improve reconstruction results. The sparsity of the scene and the structure therein is leveraged to obtain a clean image from few measurements. Throughout this chapter, perfect knowledge of the room geometry is assumed and suppression of any wall or corner returns is required.

Section 4.1 motivates sparsity-based multipath exploitation for TWRI and highlights the issues of indirect propagation paths when using existing imaging methods. Before turning to CS-based methods, Section 4.2 briefly reviews conventional image formation techniques. In Section 4.3, reconstruction of purely stationary scenes is discussed. An existing CS algorithm for multipath free environments is contrasted with two proposed multipath exploitation algorithms. Moving targets are included in the reconstruction process in Section 4.4. The general effect of target motion on multipath is discussed and two methods are proposed that exploit multipath to recover the locations and velocities of indoor targets. An extended radar geometry using multiple modules is considered in Section 4.5. The impact of co-located or distributed radar module configurations on reconstruction guarantees for the CS algorithm is discussed. Each of the three aforementioned sections is concluded with a presentation of simulation and/or experimental results. Some concluding remarks are made in Section 4.6.

The material presented in this chapter is partly taken from [LAAZ13a, LAAZ14b, LAAZ14c, LAAZ14d, LZ14, LAAZ15d, LAAZ15a, LAAZ15c, LAAZ15b].

\subsection{Motivation}

As described in the previous chapter, TWRI systems usually operate in a rich multipath environment. Due to the various indirect propagation paths, many additive signal components from the same target are received. The effect of this is that part of the targets' energy is focused at locations that do not correspond to the true locations. This phenomenon is referred to as ghost targets or simply ghosts [DL09]. A major problem in interpreting the reconstructed image is that ghosts can easily be confused 


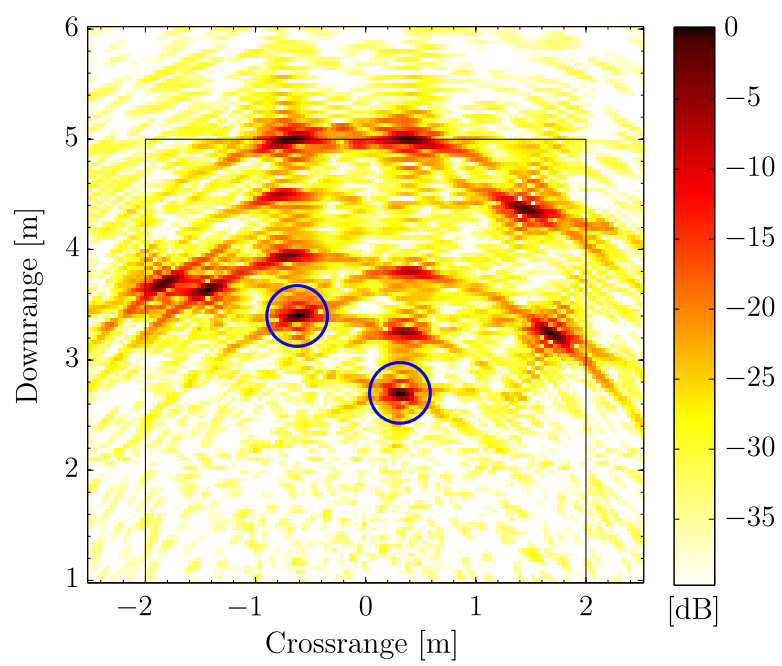

Figure 4.1. Conventional imaging result including multipath ghosts.

with real targets. This is illustrated for a scene with two targets (circled) and five indirect propagation paths in Figure 4.1. Observe that each target is accompanied by five ghost targets that closely resemble the targets of interest in the image. Hence, it is paramount to suppress or mitigate multipath effects. This idea can be taken a step further by exploiting the multipath response, as information on the target is contained in the indirect returns.

Existing multipath exploitation methods operate directly on the image [SAA11, SAN13] by projecting the ghost onto the true target location. The proposed methods utilize the additive received signal model to infer the locations of the actual targets. CS is applied to solve the inverse problem. By leveraging the sparsity of the scene, i.e., only few targets exist in the room, a unique image reconstruction can be found. CS also enables an efficient sensing scheme, such that samples can be recorded at a rate significantly lower than the Nyquist rate.

The proposed methods are extended to the reconstruction of moving targets. Also, multiple distributed radar modules are considered. As the emphasis lies on the recovery of targets, it is assumed that any non-target returns, such as wall and corner returns, have been suppressed in the received signal. Chapter 5 deals with the mitigation and suppression of wall returns.

\subsection{Conventional Image Formation}

Conventional image formation for TWRI is carried out using backprojection or Delayand-Sum Beamforming (DSBF) [Sou99, AFKA04, AAK05b]. This is applicable for 
stationary scenes and can easily be applied to the frequency domain model (3.9). The complex image value $I_{p}^{\mathrm{FD}}$ at the $p$-th spatial grid point $\left(x_{p}, y_{p}\right)$ is obtained by summing phase shifted copies of the $M N L$ signals [AAK05b],

$$
I_{p}^{\mathrm{FD}}=\frac{1}{M N L} \sum_{m=0}^{M-1} \sum_{n=0}^{N-1} \sum_{l=0}^{L-1} z_{\mathrm{FD}}[l, m, n] \exp \left(j 2 \pi f_{l} \tau_{p m n}\right), \quad p=0, \ldots P_{x} P_{y}-1,
$$

where $\tau_{p m n}$ is the focusing delay for the $m$-th transmitter, $n$-th transceiver and the $p$-th grid point. Intuitively, the expected phase shifts are compensated in the received signal and then summed over all frequencies and array elements. This can be equivalently expressed as a matrix-vector product

$$
\hat{\boldsymbol{\sigma}}=\boldsymbol{\Psi}_{\mathrm{FD}}^{H} \boldsymbol{z}_{\mathrm{FD}}
$$

where $(\cdot)^{H}$ denotes the Hermitian transpose and $\hat{\boldsymbol{\sigma}}$ is an estimate of the target state vector in space, or simply the image. Operation (4.2) is the adjoint of (3.10).

In the case of UWB radar and moving targets, standard backprojection cannot be used as moving targets will be blurred and possibly mislocated. However, DSBF can be performed for each slow time index $k$ individually. The complex image value $I_{p}(k)$, corresponding to slow time index $k$ is obtained as [AAK05b],

$$
I_{p}(k)=\left.\frac{1}{M N} \sum_{m=0}^{M-1} \sum_{n=0}^{N-1} z_{m n k}\left(t+\tau_{p m n}(k)\right) * s^{*}(-t)\right|_{t=0}, \quad p=0,1, \ldots, P_{x} P_{y}-1 .
$$

Delayed copies of the $M N$ received signals corresponding to the $k$-th pulse are summed up. Subsequently, they are fed into a matched filter with impulse response $s^{*}(-t)$ and sampled at $t=0$.

An overall image cannot be obtained by simply combining $I_{p}(k), k=0,1, \ldots, K-1$ coherently for the above stated reasons. Instead, the linear velocity model is to be included in the beamforming approach. The discrete 4D target space, described in Section 3.1, is used to take both location and velocity into account. Hence, an image value $I_{p}$ at the $p$-th space-velocity grid point $\left(x_{p}, y_{p}, v_{x p}, v_{y p}\right)$ is obtained by a summation over $K$ pulses

$$
I_{p}=\left.\frac{1}{K M N} \sum_{k=0}^{K-1} \sum_{m=0}^{M-1} \sum_{n=0}^{N-1} z_{m n k}\left(t+\tau_{p m n}(k)\right) * s^{*}(-t)\right|_{t=0}, \quad p=0,1, \ldots, P_{x} P_{y} P_{v}-1 .
$$

$\tau_{p m n}(k)$ is the focusing delay for the $(m, n)$-th transmitter-receiver pair and the $p$-th space-velocity grid point for the $k$-th pulse. The result can be regarded as $N_{v} 2 \mathrm{D}$ 
spatial images, each of which is matched to a particular velocity vector. The velocity matched beamforming in (4.4) is equivalently expressed as the adjoint of (3.6)

$$
\hat{\boldsymbol{\sigma}}=\Psi^{H} \boldsymbol{z},
$$

where $\hat{\boldsymbol{\sigma}}$ contains the 4D space-velocity information of the targets. Fast methods based on the Non-Equispaced Fast Fourier Transform (NFFT) [Bey95, DR95, PST01] for computation of (3.6) and (4.5) have been proposed in [LZ11].

The DSBF resolution is limited by the point spread function or Rayleigh resolution [RSH10]. For a linear array, the crossrange resolution reads

$$
\delta_{x}=\frac{R_{\mathrm{d}} c}{2 f_{c} L_{\text {array }}},
$$

where $R_{\mathrm{d}}$ is the downrange and $L_{\text {array }}$ is the array length. The downrange resolution is

$$
\delta_{y}=\frac{c}{2 \Delta f}
$$

where $\Delta f$ is the bandwidth of the transmitted signal.

There are two fundamental limitations of conventional image formation in the context of efficient sampling and multipath environments. First, beamforming is only applicable to the case where the full measurement data is available. Missing or undersampled data leads to a severely degraded image quality. Second, additional signal components due to multipath are not accounted for in the DSBF approach. Thus, conventional image formation leads to unwanted ghost targets as illustrated in the previous section. Ghost targets can only be dealt with in the image space as proposed in [SAA11]. These two issues are elegantly tackled by utilizing the CS framework. On the one hand, CS has be designed to recover highly resolved images of the scene from few measurements. On the other hand, the multipath propagation model (3.16) can be directly incorporated in the CS formulation to exploit multipath returns and suppress ghosts. This is discussed in the following sections.

\subsection{Stationary Targets}

First, the case of stationary targets is considered, hence, stepped-frequency measurements are assumed. Using the reduced data model in (3.23), the image formation process is cast into a sparse reconstruction problem. Note that UWB radar can 
be used likewise. In case of stationary targets the pulses can simply be accumulated coherently to improve SNR. The main results of this section were published in [LAAZ13a, LAAZ14b].

For notational convenience, all unknown vectors in (3.23) are stacked to form a single tall vector

$$
\tilde{\boldsymbol{\sigma}}=\left[\left(\boldsymbol{\sigma}^{(0)}\right)^{T}\left(\boldsymbol{\sigma}^{(1)}\right)^{T} \cdots\left(\boldsymbol{\sigma}^{(R-1)}\right)^{T}\right]^{T} \in \mathbb{C}^{P_{x} P_{y} R \times 1} .
$$

The reduced measurement vector $\overline{\boldsymbol{z}}_{\mathrm{FD}}$ can then be expressed as

$$
\overline{\boldsymbol{z}}_{\mathrm{FD}}=\boldsymbol{\Phi}_{\mathrm{FD}} \tilde{\boldsymbol{\Psi}}_{\mathrm{FD}} \tilde{\boldsymbol{\sigma}}+\boldsymbol{n}_{\mathrm{FD}}
$$

where the new dictionary matrix has now the form $\tilde{\boldsymbol{\Psi}}_{\mathrm{FD}}=\left[\begin{array}{llll}\boldsymbol{\Psi}_{\mathrm{FD}}^{(0)} & \Psi_{\mathrm{FD}}^{(1)} & \cdots & \Psi_{\mathrm{FD}}^{(R-1)}\end{array}\right] \in$ $\mathbb{C}^{L M N \times P_{x} P_{y} R}$.

\subsubsection{Conventional Sparse Reconstruction}

Before turning to multipath-aware reconstruction methods, conventional sparse reconstruction as proposed in [YA08] shall be briefly explained. This method is later used as a benchmark to show the benefits of multipath exploitation. For this approach, no multipath is considered in the CS method, i.e., only a single path $R=1$, namely the direct path is assumed to contribute to the received signal. As the scene consists of few point targets in a large $2 \mathrm{D}$ space, one can conclude that the image $\boldsymbol{\sigma}^{(0)}$ is sparse. This leads to a formulation of the sparse reconstruction problem as

$$
\hat{\boldsymbol{\sigma}}^{(0)}=\arg \min _{\boldsymbol{\sigma}^{(0)}} \frac{1}{2}\left\|\overline{\boldsymbol{z}}_{\mathrm{FD}}-\boldsymbol{\Phi}_{\mathrm{FD}} \boldsymbol{\Psi}_{\mathrm{FD}}^{(0)} \boldsymbol{\sigma}^{(0)}\right\|_{2}^{2}+\lambda\left\|\boldsymbol{\sigma}^{(0)}\right\|_{1},
$$

where $\lambda$ is the so-called regularization parameter. As multipath propagation is not considered in (4.10), the imaging result is expected to be severely affected by multipath effects. In particular, ghosts will appear in the image due to the additional propagation paths. Hence, a multipath-aware CS method should be developed to mitigate these effects.

\subsubsection{Group Sparse Reconstruction}

The goal is to exploit the full information contained in multipath, hence, the complete signal model (4.9) should be utilized. Instead of reconstructing the image corresponding to the direct path only, the complete target reflectivity vector $\tilde{\boldsymbol{\sigma}}$ must be recovered. In 


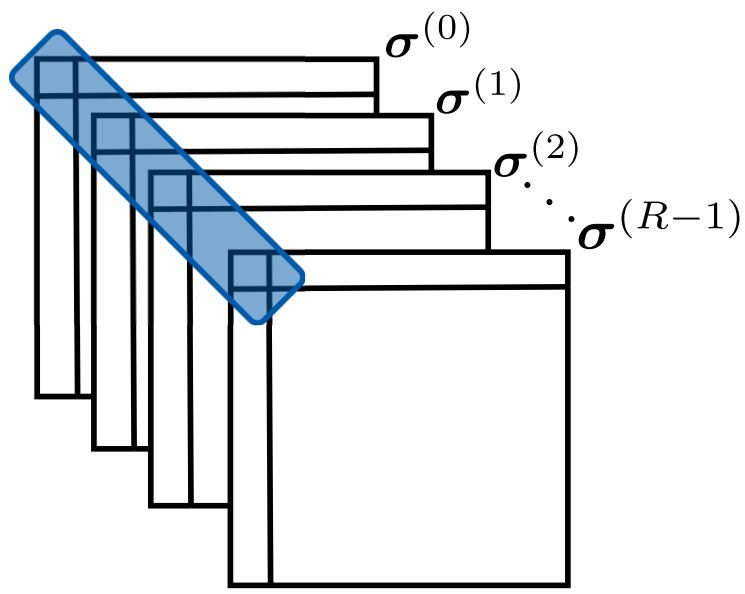

Figure 4.2. Group sparse structure for the sub-images.

practical scenarios, the scatterers are non-isotropic, i.e., the magnitude and phase of the reflectivities change with aspect angle and bistatic angle. Thus, the exact relationship between the sub-images $\boldsymbol{\sigma}^{(r)}$ corresponding to paths $r=0, \ldots, R-1$ is unknown beforehand. However, there is some prior knowledge that can be exploited. All subimages $\boldsymbol{\sigma}^{(0)}, \boldsymbol{\sigma}^{(1)}, \ldots, \boldsymbol{\sigma}^{(R-1)}$ describe targets at the same locations in the underlying sparse scene. Provided that no propagation paths are blocked, a target that is observed through one path is also observable through all other paths. Thus, a particular target will populate the same location in any of the sub-images. This means that the support of the $R$ sub-images is equal. Even if some paths are blocked or below the noise level for some scatterers, the support is at least approximately equal. This property gives rise to a particular sparsity structure of the unknown vector $\boldsymbol{\sigma}$. The sub-images exhibit a group sparse or block sparse structure, where the individual groups extend across the paths for each pixel, as illustrated in Figure 4.2. The image vectors $\boldsymbol{\sigma}^{(r)}$ are depicted as image matrices for illustration purposes.

In the next step, model (4.9) and group sparsity are exploited to reconstruct the unknown reflectivity vectors or images. It has been shown that a group sparse reconstruction can be obtained by a mixed $\ell_{2} / \ell_{1}$-norm regularization [YL06, WNF09, DYZ11, $\mathrm{BCDH10]}$. The reconstruction problem is posed as

$$
\hat{\tilde{\boldsymbol{\sigma}}}=\arg \min _{\tilde{\boldsymbol{\sigma}}} \frac{1}{2}\left\|\overline{\boldsymbol{z}}_{\mathrm{FD}}-\boldsymbol{\Phi}_{\mathrm{FD}} \tilde{\boldsymbol{\Psi}}_{\mathrm{FD}} \tilde{\boldsymbol{\sigma}}\right\|_{2}^{2}+\lambda\|\tilde{\boldsymbol{\sigma}}\|_{2,1}
$$

where

$$
\|\tilde{\boldsymbol{\sigma}}\|_{2,1}:=\sum_{p=0}^{P_{x} P_{y}-1}\left\|\left[\sigma_{p}^{(0)}, \sigma_{p}^{(1)}, \ldots, \sigma_{p}^{(R-1)}\right]^{T}\right\|_{2}=\sum_{p=0}^{P_{x} P_{y}-1} \sqrt{\sum_{r=0}^{R-1} \sigma_{p}^{(r)}\left(\sigma_{p}^{(r)}\right)^{*}}
$$


The convex optimization problem (4.11) can be solved using SparSA [WNF09] or any other available scheme [EKB10, BCDH10, DYZ11, YLY13]. Note that the number of unknowns has been multiplied by a factor of $R$ as compared to (4.10). However, by imposing the structured sparse constraint, the degrees of freedom of the problem is reduced to remedy the impact of the increased problem dimension. Hence, a lower number of measurements may be sufficient for accurate reconstruction as compared to non-structured regularization [DYZ11]. Thus, the recovery performance is improved by the group sparse problem formulation.

Once a solution $\hat{\tilde{\boldsymbol{\sigma}}}$ is obtained, the sub-images are combined to form an overall image. Since the phase relationship between the individual sub-images is generally unknown, coherent combination is not feasible. Thus, the sub-images are accumulated incoherently by taking the $\ell_{2}$-norm over each group

$$
\left[\hat{\boldsymbol{\sigma}}_{\mathrm{GS}}\right]_{p}=\left\|\left[\sigma_{p}^{(0)}, \sigma_{p}^{(1)}, \ldots, \sigma_{p}^{(R-1)}\right]^{T}\right\|_{2}, p=0, \ldots, P_{x} P_{y}-1 .
$$

The incoherent combination of the sub-images does not improve the SNR, as spatially white noise will also be accumulated. However, the Signal-to-Clutter Ratio (SCR) of the final image is improved. On the one hand, clutter caused by ghost targets is largely suppressed as the multipath returns are accounted for in the model formulation. On the other hand, if residual clutter remains in the reconstruction, caused e.g. by propagation paths or physical effects not accounted for in the model, it is attenuated in the final image. It is expected that residual clutter is spatially non-white and independently distributed with respect to the sub-images. Hence, after incoherent combination, these residuals are averaged out in the results as they do not align across the sub-images. Note that the performance of this approach relies on sufficient power in the multipath returns. If the multipath returns are very weak, they cannot contribute to an improvement in the image and should be neglected.

There are two challenges when dealing with practical scenarios. First, in order to achieve good recovery performance, just the significant multipath paths should be included in the model. Neglecting significant paths leads to remaining ghost targets and, thus, increased clutter in the final image. Including too many paths results in an unnecessary increase of the number of unknowns and, thereby, the CS reconstruction performance will drop. Hence, the significant paths must be inferred from prior knowledge of the building layout. Second, the precise knowledge of locations of the interior wall is very important. All returns via one particular path are coherently processed. Hence, an error in an interior wall location has the same effect as an error in the array element positions. Further, the target positions, as viewed via different paths, only overlap if the wall location errors are sufficiently low. Thus, the group sparse property 
is lost if the errors are too large. The problem of inaccuracies in the interior wall positions will be discussed later in Section 5.3.

\subsubsection{Sparse Reconstruction With Overlapping Groups}

The above described group sparse model can be generalized to overlapping groups. Instead of using non-overlapping groups corresponding to a single pixel in each subimage, the groups are extended to a neighborhood around each pixel. This is motivated by the observation that, in high-resolution images, targets are not perfect points but rather occupy a number of adjacent pixels. The extent of the target in the image depends on its dimension and is also a function of the imaging system resolution in range and cross-range. This prior neighborhood information is incorporated into the group sparse constraints. Additionally, an intra-group weighting is introduced to allow for a certain "tapering" of the groups. Towards this end, the mixed-norm term (4.12) is replaced by the regularizer,

$$
\zeta(\tilde{\boldsymbol{\sigma}}):=\sum_{p=0}^{P_{x} P_{y}-1} \sqrt{\sum_{r=0}^{R-1}\left\|\boldsymbol{W}^{(p)} \sigma_{g_{p}}^{(r)}\right\|_{2}^{2}},
$$

where $g_{p} \subseteq\left\{0,1, \ldots, R P_{x} P_{y}-1\right\}$ is an index set corresponding to the group of pixels forming a neighborhood around pixel $p$. The diagonal weighing matrix $\boldsymbol{W}^{(p)} \in \mathbb{R}^{\left|g_{p}\right| \times\left|g_{p}\right|}$ ensures that the weighting within a group is according to the desired pixel neighborhood relation. This yields the convex optimization problem

$$
\hat{\tilde{\boldsymbol{\sigma}}}=\arg \min _{\tilde{\boldsymbol{\sigma}}} \frac{1}{2}\left\|\overline{\boldsymbol{z}}_{\mathrm{FD}}-\boldsymbol{\Phi}_{\mathrm{FD}} \tilde{\boldsymbol{\Psi}}_{\mathrm{FD}} \tilde{\boldsymbol{\sigma}}\right\|_{2}^{2}+\lambda \zeta(\tilde{\boldsymbol{\sigma}}) .
$$

Figure 4.3 illustrates the overlapping grouping of image pixels assuming an $P_{x} \times P_{y}=$ $10 \times 10$ pixel image and $R=2$ possible propagation paths. The small number in the top left corner of each square indicates the pixel index, while the large number in the center represents the pixel weights of the depicted group. For example the index set for the group corresponding to the 12th pixel, as shown in the figure, would be $g_{12}=\{2,11,12,13,22,102,111,112,113,122\}$, where the second half of the set corresponds to indices in the multipath image $(r=1)$. The corresponding weighing matrix would be $\boldsymbol{W}^{(12)}=\frac{1}{2} \operatorname{diag}\left(\frac{1}{8}, \frac{1}{8}, \frac{1}{2}, \frac{1}{8}, \frac{1}{8}, \frac{1}{8}, \frac{1}{8}, \frac{1}{2}, \frac{1}{8}, \frac{1}{8}\right)$. The index sets for all other groups are obtained similarly by shifting the cross-shaped mask to the appropriate pixel position. The weights should be chosen such that their sum is unity in order to avoid unintentional scaling of the reconstruction result.

Note that the regularizer (4.14) in reconstruction problem (4.15) is not separable. That is, it cannot be expressed as a summation of functions of disjoint sets of its variables, 


$$
r=0
$$

\begin{tabular}{|c|c|c|c|c|}
\hline 1 & ${ }^{11} \frac{1}{8}$ & 21 & & 91 \\
\hline $\begin{array}{ll}2 & \frac{1}{8} \\
& \end{array}$ & ${ }^{12} \frac{1}{2}$ & ${ }^{22} \frac{1}{8}$ & & 92 \\
\hline 3 & ${ }^{13} \frac{1}{8}$ & 23 & & 93 \\
\hline & & & & \\
\hline 10 & 20 & 30 & & 100 \\
\hline
\end{tabular}

$r=1$

\begin{tabular}{|c|c|c|c|c|}
\hline 101 & ${ }^{111} \frac{1}{8}$ & 121 & & 191 \\
\hline${ }^{102} \frac{1}{8}$ & ${ }^{112} \frac{1}{2}$ & ${ }^{122} \frac{1}{8}$ & & \begin{tabular}{|l|}
192 \\
\end{tabular} \\
\hline 103 & ${ }^{113} \frac{1}{8}$ & 123 & & 193 \\
\hline & & & & \\
\hline 110 & 120 & 130 & & 200 \\
\hline
\end{tabular}

Figure 4.3. Illustration of a first-order neighborhood grouping. The left image corresponds to the direct path and the right image corresponds to multipath.

and thus, cannot be solved by SparSA [WNF09]. However, FoGLasso [YLY13] allows for overlapping groups and is applied to the generalized group sparse reconstruction problem.

\subsubsection{Simulation and Experimental Results}

For both simulation and experiments, the same measurement setup and room layout is used as depicted in Figure 4.4. A 77-element uniform linear monostatic array with an inter-element spacing of $1.9 \mathrm{~cm}$ is used for imaging. The origin of the coordinate system is chosen to be at the center of the array. The concrete front wall is located parallel to the array at $2.44 \mathrm{~m}$ downrange and has a thickness $d_{\text {wall }}=20 \mathrm{~cm}$ and relative permittivity $\varepsilon_{\mathrm{r}}=7.6632$. The left sidewall is at a crossrange of $-1.83 \mathrm{~m}$, whereas the back wall resides at $6.37 \mathrm{~m}$ downrange. Also, there is a protruding corner on the right at $3.4 \mathrm{~m}$ crossrange and $4.57 \mathrm{~m}$ downrange. A stepped-frequency signal, consisting of $M=801$ equally spaced frequency steps covering the 1 to $3 \mathrm{GHz}$ band is employed for scene interrogation.

\section{Simulation Results}

Two point targets, located at coordinates $(0.31,3.6) \mathrm{m}$ and $(-0.62,5.2) \mathrm{m}$, are simulated using the target-only model (3.23). The region of interest is discretized into $P_{x} \times P_{y}=$ $64 \times 64$ pixels. In total, $R=5$ round-trip propagation paths are considered, which are composed of the direct path and a one-way path corresponding to direct, back wall 


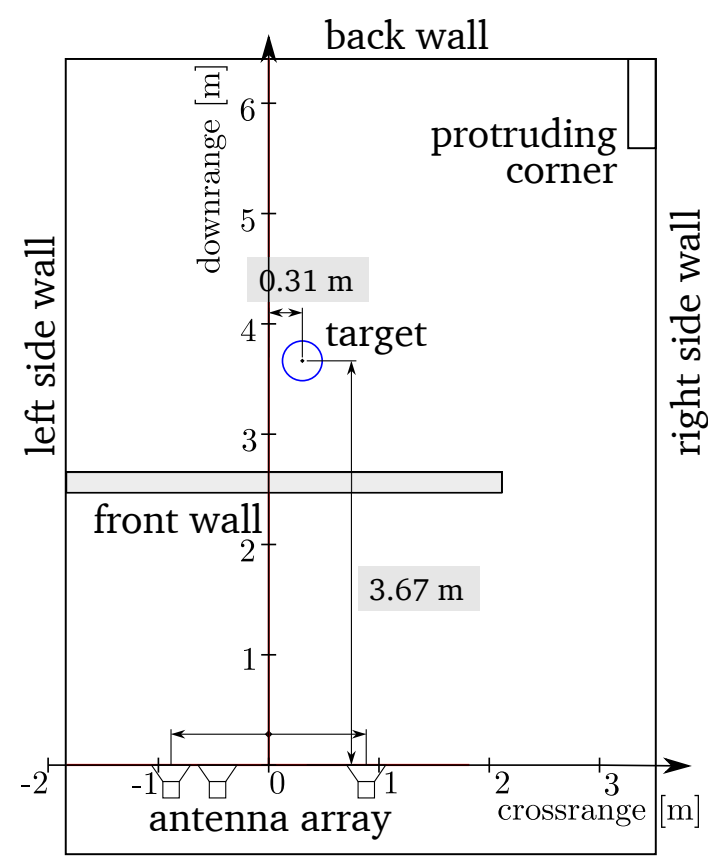

Figure 4.4. Measurement setup and room layout.

multipath, left side wall multipath, multipath w.r.t. the protruding right corner, and the wall ringing multipath. Global path weights are chosen to account for additional losses due to secondary reflection, which are set to $1,1,0.3,0.5$ and 0.4 , respectively. White noise with $0 \mathrm{~dB}$ SNR is added to the simulated measurements.

For comparison, the beamformed image using the full data record is depicted in Figure $4.5 \mathrm{a}$. Observe that conventional beamforming is not able to deal with multipath. Hence, a total number of eight ghosts are reconstructed along with the two targets. For the CS results in Figure 4.5, only one-fourth of the array elements and one-eighth of the frequencies are used for scene reconstruction. Subsets of both the array elements and frequencies bins are chosen at random. The results are averaged over 100 Monte Carlo runs. Figure 4.5b shows the reconstruction result using a conventional CS approach, which does not exploit multipath [YA08]. It is observed that the true targets are reconstructed along with all ghost targets, resulting in a highly cluttered scene. The group sparse reconstruction approach, shown in Figure 4.5c, provides a superior performance. All ghost targets have been suppressed while the two correct targets remain perfectly visible. Overlapping groups result in a smoother depiction of the targets, as shown in Figure 4.5d. 


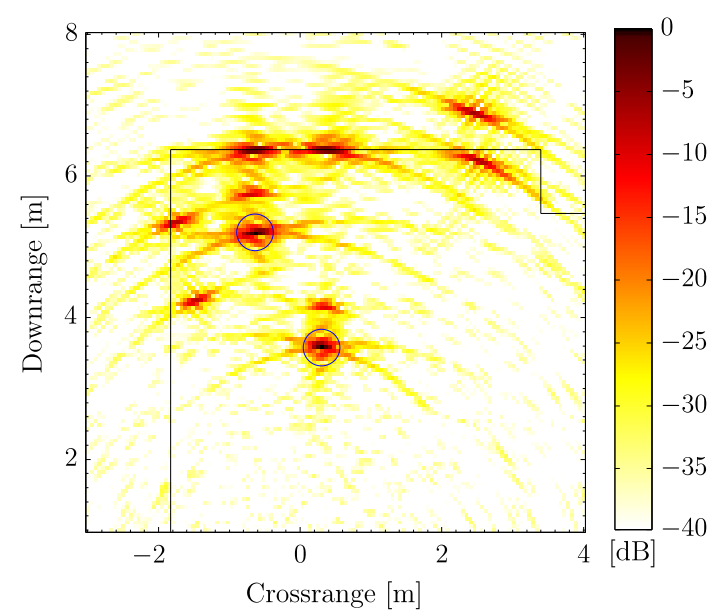

(a) Conventional DSBF

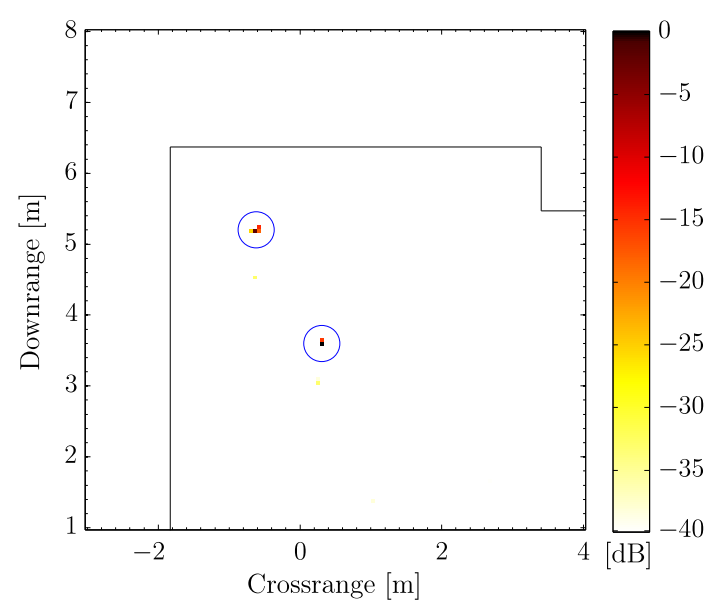

(c) Group sparse CS

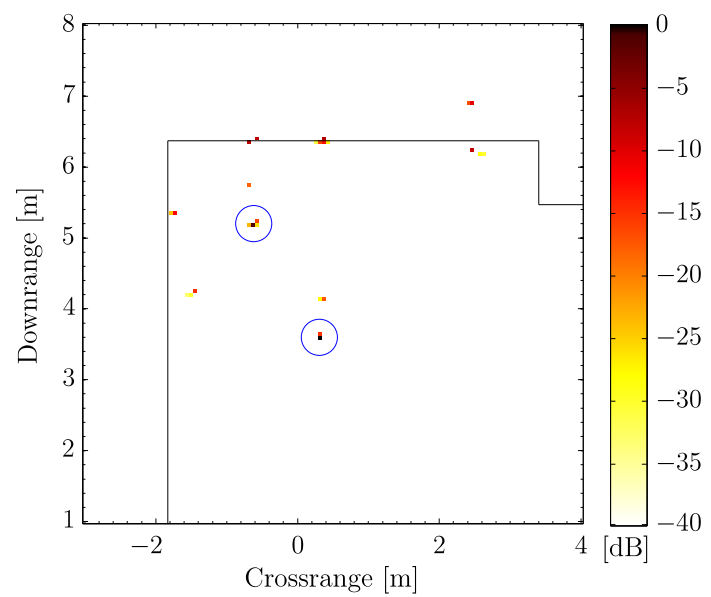

(b) Conventional CS

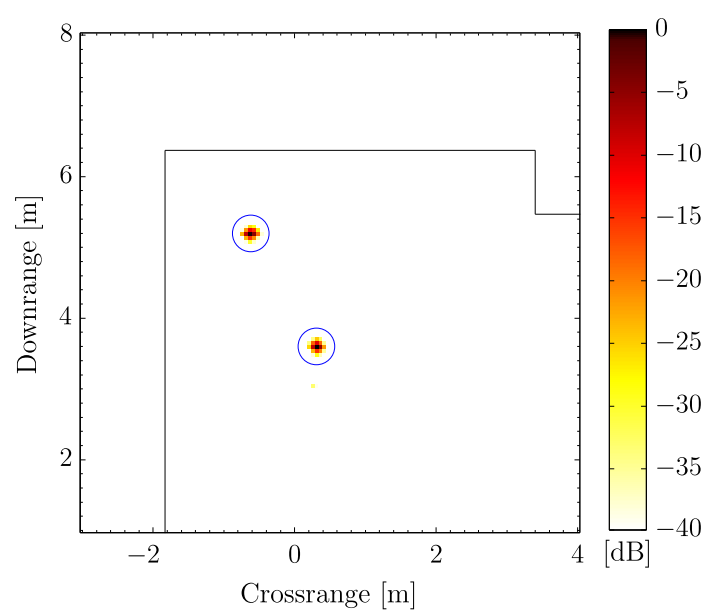

(d) Overlapping group sparse CS

Figure 4.5. Reconstruction results using different algorithms for the simulated scene with two point targets. One fourth of the array elements and one eighths of the frequency bins were used for the formation of (b) - (d). Image (a) was created using the full set of measurements.

\section{Experimental Results}

Experiments were conducted in a semi-controlled lab environment at the Radar Imaging Lab, Villanova University providing real data. A single aluminum (Al) pipe $(61 \mathrm{~cm}$ long, $7.6 \mathrm{~cm}$ diameter) was placed upright on a $1.2 \mathrm{~m}$ high foam pedestal at $3.67 \mathrm{~m}$ downrange and $0.31 \mathrm{~m}$ crossrange. Compared with the simulation scenario, the left and right side walls were covered with Radio Frequency (RF) absorbing material, but the protruding right corner and the back wall were left uncovered. Background subtraction [AA08a, $\left.\mathrm{MKA}^{+} 08\right]$ has been performed on the data, as the aim is to focus on target multipath only. Figure 4.6a depicts the beamformed image using all available data. 


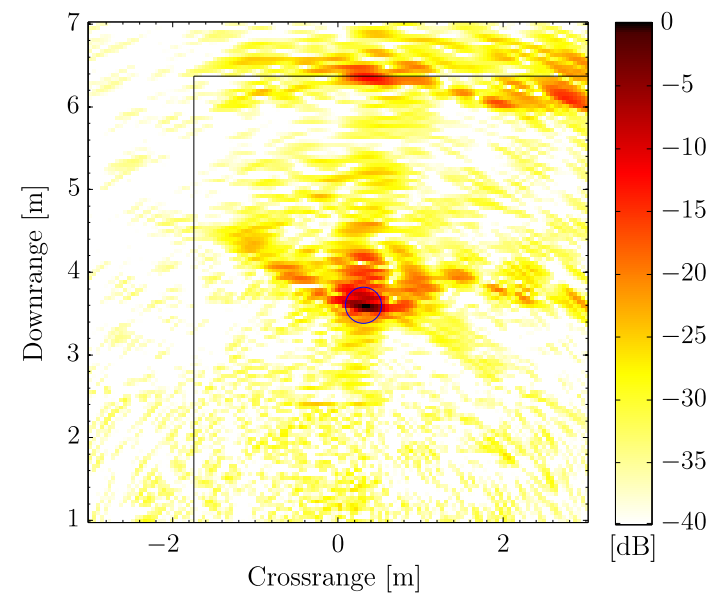

(a) Conventional DSBF

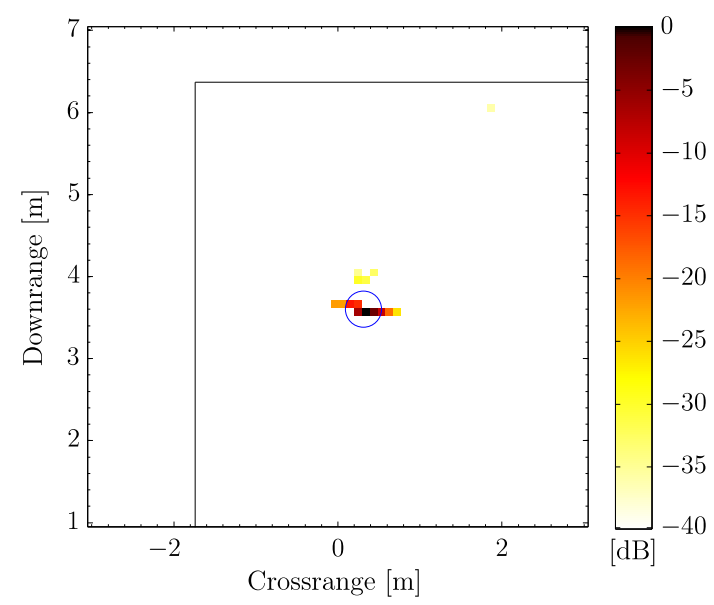

(c) Group sparse CS

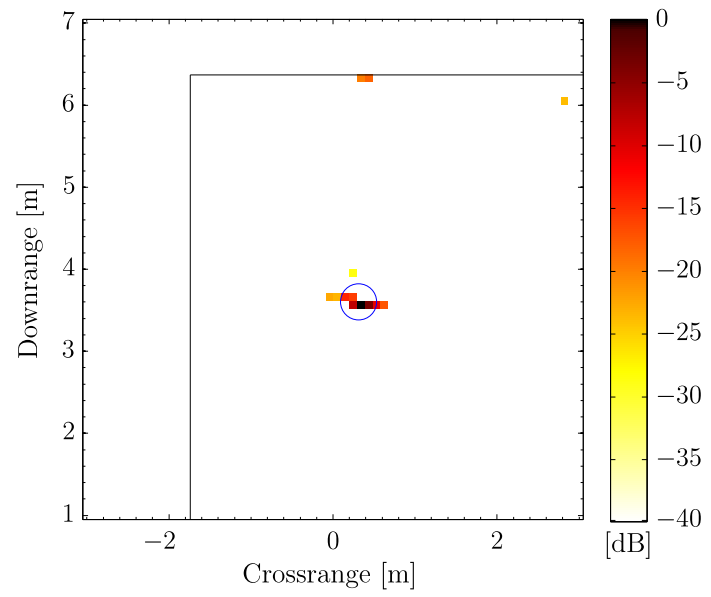

(b) Conventional CS

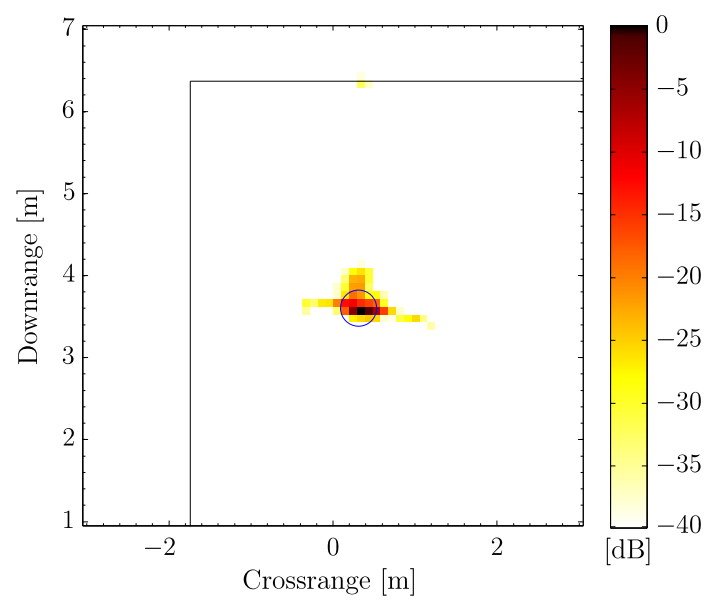

(d) Overlapping group sparse CS

Figure 4.6. Imaging results using different algorithms for the $\mathrm{Al}$ pipe scene. Conventional DSBF reconstruction using full measurements is shown in (a). CS reconstruction using one fourth of the array elements and half of the frequency bins is depicted in (b) - (d).

When comparing with our simulated data, we can conclude that only the multipath ghosts, due to the back wall, and the protruding corner in the back are visible. Hence, only the direct path and two multipaths are considered for group sparse reconstruction. As other paths appear too weak, it is better to neglect them in the model. One-fourth of the array elements and one-half of the frequencies are used for sparse reconstruction. The conventional CS result is shown in Figure 4.6b, where the multipath ghost from the back wall is still visible. The group sparse reconstruction approach with multipath exploitation is capable of suppressing this ghost, see Figure 4.6c. Observe the smoother depiction of the target in Figure 4.6d when using overlapping groups. 


\subsection{Stationary and Moving Targets}

In the previous section, purely stationary scenes have been considered. Extending this approach, moving targets are now included in the scene of interest. Hence, both the location and the velocity of indoor targets shall be recovered. This requires additional dimensions in both the measurements and the image as compared to the purely stationary case. Clearly, the unknown vector must now include the target velocities. All possible target states are discretized on two spatial and two velocity dimensions, as described in Section 3.1. By using an UWB pulse radar, another dimension in the measured signal arises, i.e., the slow time index $k$. In order to resolve target movements, $K$ pulses are transmitted and coherently processed. Note that only instantaneous reconstruction of target locations and velocities is treated in this thesis, i.e., tracking of targets is not considered. The major findings of this section were published in [LAAZ14c, LAAZ15b, LAAZ15a].

\subsubsection{Apparent Doppler Speed}

Before turning to the reconstruction methods, the effect of multipath in conjunction with moving targets is considered. It is shown that multipath returns contain additional information on target motion that can be exploited in the reconstruction. A target at initial position $\left(x_{p}, y_{p}\right)$ moving with uniform velocity $\left(v_{x p}, v_{y p}\right)$ exhibits different apparent Doppler speeds when observed via different propagation paths. The scalar apparent Doppler speed is the component of the target velocity that can be observed via the Doppler shift and is associated with a specific propagation path. In case of direct propagation and a monostatic setup, this is the radial velocity component with respect to the location of the transceiver. Intuitively, in a monostatic radar, the locus of constant range is a circle which is also the trajectory of zero Doppler. Thus, only velocities perpendicular to this circle, i.e., radial velocities, can be observed. For a bistatic setup, the trajectory of zero Doppler forms an ellipse with the interrogating transmitter/receiver pair as foci and the observable velocity component is orthogonal to this ellipse.

If the transmitted pulse travels along an indirect path, the apparent Doppler speed changes. In order to illustrate the apparent Doppler speed under multipath propagation, an alternate transmitter/receiver/target geometry is considered. In the case of multipath, the physical transmitter and/or receiver locations can be reflected about the secondary reflector (interior wall). For first order multipath, either the transmitter 


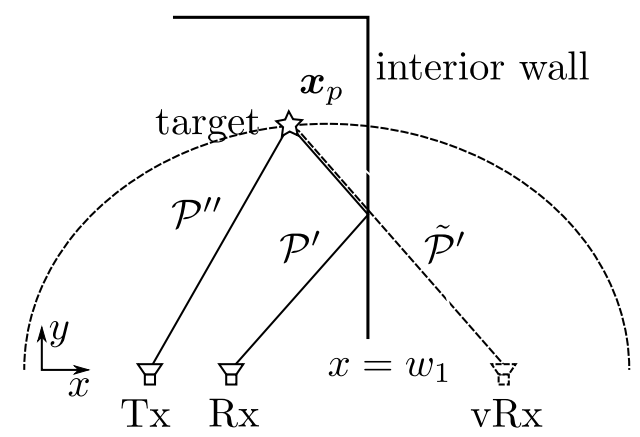

Figure 4.7. Alternate multipath geometry with a virtual receiver and the corresponding constant range ellipse.

or receiver location is mirrored depending on whether the secondary reflection occurs on transmit or receive. Conversely, both the transmitter and receiver locations will be mirrored for second order quasi-monostatic multipath propagation. The mirrored locations constitute a virtual transmitter and a virtual receiver. Multipath propagation can now be cast as direct propagation to/from these virtual antenna locations. As such, multipath can be lead back to a simple bistatic radar setup. Hence, the apparent Doppler speed can be determined as the normal velocity component with respect to the ellipse formed by the virtual transmitter/receiver pair. In other words, the observed Doppler speed of the target under multipath propagation is the projection of the target velocity onto this normal. An example scenario is depicted in Figure 4.7, where multipath occurs on the return path only. The signal travels along path $\mathcal{P}^{\prime \prime}$ from the physical transmitter $(\mathrm{Tx})$ to the target and along path $\mathcal{P}^{\prime}$ back to the physical receiver $(\mathrm{Rx})$ via reflection at the interior wall. The return path can equivalently be described by direct propagation along path $\tilde{\mathcal{P}}^{\prime}$ to a virtual receiver $(\mathrm{vRx})$ that has been constructed as described above. Now, the normal to the ellipse formed by the physical transmitter (Tx) and the virtual receiver (vRx) needs to be considered for the apparent Doppler speed.

Instead of using the geometric interpretation, this speed may also be approximated using propagation delays. Depending on the transmitter $m$, the receiver $n$, the path $r$, and averaging over the full CPI, the apparent Doppler speed for the $p$-th target may be expressed as

$$
v_{D, p m n}^{(r)}=\frac{1}{K-1} \sum_{k=0}^{K-2} c \frac{\tau_{p m n}^{(r)}(k+1)-\tau_{p m n}^{(r)}(k)}{T_{\mathrm{r}}} .
$$

Hence, for each target, $R$ apparent Doppler speeds are obtained, which are the projections of the target velocity vector onto the respective normal directions. This additional diversity in the multipath returns is exploited to obtain an estimate of the full target velocity vector. In the sequel, small arrays are assumed, such that the apparent Doppler 


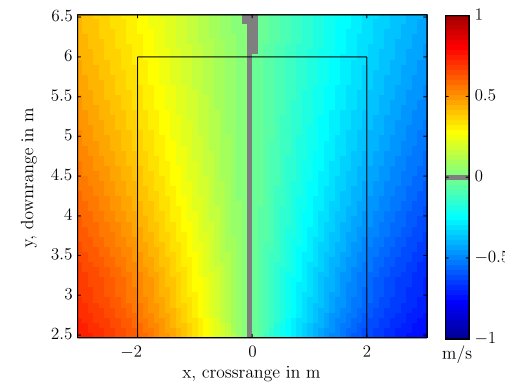

(a) Direct path

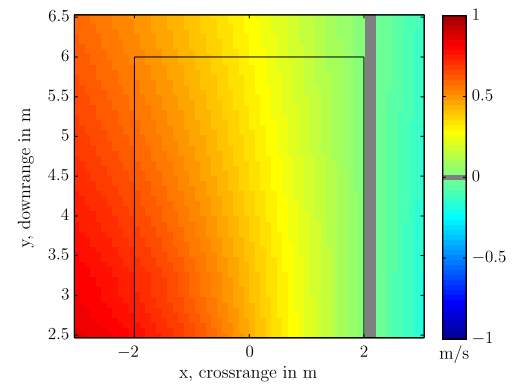

(b) First order multipath via the right side wall

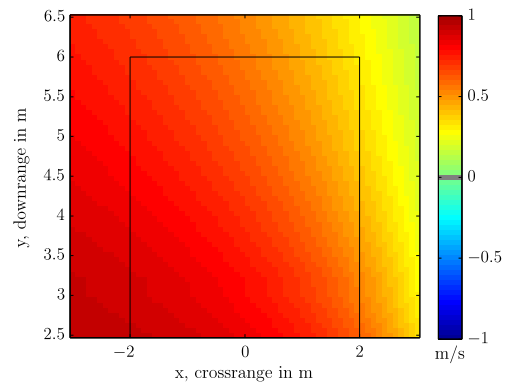

(c) Second order multipath via the right side wall

Figure 4.8. Apparent Doppler speed for a target moving with velocity $(1,0) \mathrm{m} / \mathrm{s}$.

speed does not change with the transmit/receive array elements

$$
v_{D, p}^{(r)} \approx v_{D, p m n}^{(r)}, \quad m=0, \ldots, M-1, \quad n=0, \ldots, N-1
$$

Hence, $v_{D, p}^{(r)}$ only depends on the propagation path as well as the target location and velocity.

Consider the simulation of a target at an arbitrary location within a room, moving with a velocity $\left(v_{x p}, v_{y p}\right)=(1,0) \mathrm{m} / \mathrm{s}$. That is, the target is solely moving in the crossrange direction. At each assumed target position, the apparent Doppler speed is color coded in Figure 4.8. The surrounding walls are also superimposed on the figures. The speed pattern is shown for the transmitter/receiver pair located at the centers of the transmit and receive arrays. The direct propagation case is shown in Figure 4.8a. One can observe the expected pattern showing zero Doppler along the broadside and gradually increasing speed for angles deviating from broadside. However, the pattern changes for indirect paths involving reflection at the right side wall. Figure $4.8 \mathrm{~b}$ corresponds to a first order multipath that involves direct propagation on transmit and a secondary reflection on receive. In addition, Figure $4.8 \mathrm{c}$ illustrates the effect of second order quasi-monostatic multipath via the right side wall. The patterns in Figures 4.8b,c are shifted and distorted as compared to Figure 4.8a. In particular, the zero speed line is shifted as compared to that in Figure 4.8a. Hence, additional information on target motion is contained in the first and second order multipath returns. If properly modeled, as described earlier, this property can be exploited to improve the velocity estimation of targets. 


\subsubsection{Joint Target Location and Velocity Estimation}

As laid out above, there are clear benefits in exploiting multipath, especially for target velocity estimation. Hence, the CS method from Section 4.3 shall be extended to include target motion. Analogous to the definition (4.9) in the stationary target case, a measurement equation is defined including all paths from all possible targets. Using the multipath model for moving targets (3.6), the complete set of linear measurements is written as

$$
\overline{\boldsymbol{z}}=\boldsymbol{\Phi} \tilde{\boldsymbol{\Psi}} \tilde{\boldsymbol{\sigma}}+\boldsymbol{n}
$$

where $\tilde{\boldsymbol{\Psi}}=\left[\begin{array}{llll}\boldsymbol{\Psi}^{(0)} & \boldsymbol{\Psi}^{(1)} & \ldots & \boldsymbol{\Psi}^{(R-1)}\end{array}\right] \in \mathbb{C}^{M N K T \times P_{x} P_{y} P_{v} R}$ is the concatenated overcomplete dictionary for all possible paths and the unknown target state vectors are stacked into one tall vector $\tilde{\boldsymbol{\sigma}}=\left[\begin{array}{lll}\left(\boldsymbol{\sigma}^{(0)}\right)^{T} & \left(\boldsymbol{\sigma}^{(1)}\right)^{T} \cdots\left(\boldsymbol{\sigma}^{(R-1)}\right)^{T}\end{array}\right]^{T} \in \mathbb{C}^{P_{x} P_{y} P_{v} R \times 1}$.

Given the reduced measurements $\overline{\boldsymbol{z}}$ in (4.18), the target state information $\tilde{\boldsymbol{\sigma}}$ is recovered using sparse reconstruction. Joint target location and velocity estimation is a straight forward extension of the group sparse recovery of stationary targets (4.11). Again, the groups comprise all paths, however, for any target in the $4 \mathrm{D}$ target space. The reconstruction problem is formulated as a mixed $\ell_{2} / \ell_{1}$-norm minimization

$$
\hat{\boldsymbol{\sigma}}=\arg \min _{\tilde{\boldsymbol{\sigma}}} \frac{1}{2}\|\overline{\boldsymbol{z}}-\boldsymbol{\Phi} \tilde{\boldsymbol{\Psi}} \tilde{\boldsymbol{\sigma}}\|_{2}^{2}+\lambda\|\tilde{\boldsymbol{\sigma}}\|_{2,1}
$$

where

$$
\|\tilde{\boldsymbol{\sigma}}\|_{2,1}=\sum_{p=0}^{P_{x} P_{y} P_{v}-1}\left\|\left[\sigma_{p}^{(0)}, \sigma_{p}^{(1)}, \ldots, \sigma_{p}^{(R-1)}\right]^{T}\right\|_{2}
$$

As before, the convex optimization problem (4.19) can be solved for example using SparSA [WNF09] or BOMP [EKB10] which are contrasted in the sequel. SparSA obtains a near-optimum solution for (4.19) at the cost of high numerical complexity, whereas the BOMP finds a sub-optimal solution at significantly lower computational cost.

Once a solution $\hat{\boldsymbol{\sigma}}$ is obtained, a composite target state vector of the scene is obtained by non-coherent combination of the individual state vectors. The final recovery result contains the information about the location and the translatory motion of all targets in the scene. Stationary targets are included in the spatial image corresponding to the zero velocity case. 


\subsubsection{Target Location Reconstruction With Subsequent Ve- locity Estimation}

An alternative approach is splitting the reconstruction into target localization based on compressive sensing and subsequently performing velocity estimation using conventional Doppler processing. This idea was proposed by Dang and Kilic [DK14], however, no multipath was considered and, thus, only the scalar Doppler speed was recovered. By extending this two-step approach to multipath scenarios, the additional information is exploited to reconstruct the full target velocity vector.

In the target localization step, the 2D image reconstruction problem is solved for each slow time index individually. Instead of reconstructing the full 4D target state vector as in (4.19), we assume stationary targets for each processed pulse. As the returns at a single slow time do not contain any velocity information, only the spatial information of the scene is reconstructed. The scene reflectivity vectors $\tilde{\boldsymbol{\sigma}}^{(r)}(k) \in \mathbb{C}^{P_{x} P_{y}}$ now depend on the slow time $k$. The single pulse measurements $\overline{\boldsymbol{z}}_{\mathrm{SP}}(k)$ can be expressed as

$$
\overline{\boldsymbol{z}}_{\mathrm{SP}}(k)=\boldsymbol{\Phi}_{\mathrm{SP}} \boldsymbol{z}_{\mathrm{SP}}(k)=\boldsymbol{\Phi}_{\mathrm{SP}} \tilde{\boldsymbol{\Psi}}_{\mathrm{SP}} \tilde{\boldsymbol{\sigma}}(k) .
$$

The measurement vectors are formed by stacking the returns corresponding to all transmitters and receivers for each pulse as $\boldsymbol{z}(k)=\left[\boldsymbol{z}_{00 k}^{T}, \ldots, \boldsymbol{z}_{M-1 N-1 k}^{T}\right]^{T}, k=$ $0, \ldots, K-1$ from the full measurement model (3.4). The downsampling matrix $\boldsymbol{\Phi}_{\mathrm{SP}} \in \mathbb{R}^{T_{\mathrm{d}} M_{\mathrm{d}} N_{\mathrm{d}} \times T M N}$ is constructed in the same manner as described in (3.24). However, no downsampling in the slow time domain is performed. The reduced dictionary $\tilde{\mathbf{\Psi}}_{\mathrm{SP}} \in \mathbb{C}^{T M N \times R P_{x} P_{y}}$ is the part of the full dictionary $\tilde{\boldsymbol{\Psi}}$ corresponding to $k=0$ and zero target velocity while everything else is deleted. Using (4.21), for each $\overline{\boldsymbol{z}}_{\mathrm{SP}}(k)$, a scene reflectivity vector $\hat{\tilde{\boldsymbol{\sigma}}}(k)$ is reconstructed by solving the mixed-norm problems

$$
\hat{\tilde{\boldsymbol{\sigma}}}(k)=\arg \min _{\tilde{\boldsymbol{\sigma}}(k)} \frac{1}{2}\left\|\overline{\boldsymbol{z}}_{\mathrm{SP}}(k)-\boldsymbol{\Phi}_{\mathrm{SP}} \tilde{\boldsymbol{\Psi}}_{\mathrm{SP}} \tilde{\boldsymbol{\sigma}}(k)\right\|_{2}^{2}+\lambda\|\tilde{\boldsymbol{\sigma}}(k)\|_{2,1}, \quad k=0, \ldots, K-1 .
$$

The mixed-norm regularization again ensures the group sparse property as used in (4.11) and (4.19). Note that despite solving $K$ different CS problems, the corresponding computational load is much lower as the number of unknowns is greatly reduced (by the potentially large number of velocity bins $P_{v}$ ). An intermediate image containing target location reconstruction can then be formed by a non-coherent summation over all paths and pulses. At this point, a target detection step should be carried out to select only $P_{\text {sig }}$ targets with significant amplitude to reduce the computational complexity as much as possible for the subsequent velocity estimation procedure. Pixels with very low amplitude can usually be discarded as they correspond to clutter or noise. 
In the velocity estimation step of the method, the velocity vector for each target is estimated by Doppler processing of the CS reconstruction result. For each of the $P_{\text {sig }}$ targets, $K R$ complex reflectivities have been reconstructed, one for every combination of propagation path and pulse, denoted by

$$
\hat{\boldsymbol{b}}_{p}^{(r)}=\left[\hat{\sigma}_{p}^{(r)}(0), \ldots, \hat{\sigma}_{p}^{(r)}(K-1)\right]^{T} \in \mathbb{C}^{K}, p=0, \ldots, P_{\mathrm{sig}}-1, r=0, \ldots, R-1 .
$$

The apparent Doppler speed of the target causes a phase progression along the slow time dimension $k$. The slope of phase progression encodes the Doppler speed and depends on the location/velocity of the target as well as the propagation path. By taking the discrete-time Fourier transform of $\hat{\boldsymbol{b}}_{p}^{(r)}$ along the slow time, one obtains the Doppler information for the targets $\hat{B}_{p}^{(r)}(\omega)$. Assuming a single target per location cell, the apparent Doppler speed for each target and path is found as the peak in the Fourier-transformed slow time vector

$$
\hat{v}_{D, p}^{(r)}=\frac{c}{\pi f_{c}} \arg \max _{\omega} \hat{B}_{p}^{(r)}(\omega) .
$$

Hence, for each target $R$ apparent Doppler speeds are obtained corresponding to the various propagation paths. From the investigations in the above section, the apparent Doppler speeds of a target with known location can be calculated for arbitrary target velocity vectors. Hence, the inverse problem is to be solved, i.e., recovering the target velocity vector from apparent Doppler speed measurements. The problem can be formulated as solving the following linear system of equations

$$
\left[\begin{array}{c}
\hat{v}_{D, p}^{(0)} \\
\vdots \\
\hat{v}_{D, p}^{(R-1)}
\end{array}\right]=\left[\begin{array}{cc}
\left.v_{D, p}^{(0)}\right|_{v_{p}=(1,0)^{T}} & \left.v_{D, p}^{(0)}\right|_{v_{p}=(0,1)^{T}} \\
\vdots & \vdots \\
\left.v_{D, p}^{(R-1)}\right|_{v_{p}=(1,0)^{T}} & \left.v_{D, p}^{(R-1)}\right|_{v_{p}=(0,1)^{T}}
\end{array}\right]\left[\begin{array}{c}
v_{p x} \\
v_{p y}
\end{array}\right], \quad p=0, \ldots, P_{\text {sig }}-1,
$$

where $\left.v_{D, p}^{(r)}\right|_{v_{p}=(1,0)^{T}}$ and $\left.v_{D, p}^{(r)}\right|_{v_{p}=(0,1)^{T}}$ correspond to the apparent Doppler speed of the $r$-th path for a target located at the $p$-th position moving with $1 \mathrm{~m} / \mathrm{s}$ along the $x$-axis or $y$-axis, respectively. Obviously, at least $R=2$ paths are needed, such that (4.25) has a unique solution. It is advantageous to exploit more than two paths and obtain a velocity estimate $\left(\hat{v}_{p x}, \hat{v}_{p y}\right)^{T}$ from $(4.25)$ in the least-squares sense. Note that the exploitation of multipath enables an estimate of the full target velocity vector, whereas a single path only delivers a scalar Doppler speed.

The described velocity estimation method can be extended to multiple targets within a single location resolution cell. In this case, multiple apparent Doppler velocities need to be extracted from each path. This, however, results in resolution and association issues. First, multiple Doppler velocities may only be found if they are sufficiently distinct and 
can be resolved in the Fourier-transformed slow time. Second, the association of the found velocities to the targets is not obvious. In the case of few targets per cell and a few paths a combinatorial search may be feasible. That is, any possible association is attempted and the result with the lowest estimation residual is chosen as the correct velocity estimate. If the number targets in the resolution cell is unknown, a source enumeration step needs to be carried out first.

The final result of this two-step method is a reconstructed image of the scene and corresponding velocity estimates for the detected targets.

\subsubsection{Simulation and Experimental Results}

Simulation and experimental results are presented to demonstrate the effectiveness of the proposed multipath exploitation approaches for location and velocity estimation. The setups are chosen such that they represent a realistic wideband pulsed TWRI system. The multipath environment is modeled to mimic a typical room behind a concrete exterior wall.

\section{Simulation Results}

Simulations were performed for a wideband pulse-Doppler multistatic radar with a 4element uniform linear array of length $1 \mathrm{~m}$. Each array element can be used for both transmission and reception, leading to $M=N=4$. A modulated Gaussian pulse, centered around $f_{c}=2 \mathrm{GHz}$, with a relative bandwidth of $50 \%$ is transmitted. The PRI is set to $10 \mathrm{~ms}$ and $K=15$ pulses are processed coherently. At the receiving side, $T=150$ fast time samples in the relevant interval, covering the target and multipath returns, are taken at a sampling rate of $f_{s}=4 \mathrm{GHz}$. The front wall is modeled with $d_{\text {wall }}=20 \mathrm{~cm}$ thickness and relative permittivity $\varepsilon_{\mathrm{r}}=7.66$, and is located parallel to the array at a distance of $3 \mathrm{~m}$. Two side walls are considered at $\pm 2 \mathrm{~m}$ in crossrange, each of which causes 3 different multipath returns per target. These are, in total, 4 first order multipath returns and 2 second order quasi-monostatic multipath returns per target, which are all considered to be $6 \mathrm{~dB}$ weaker than the direct path. Hence, in total, there are $R=7$ paths per target contributing to the received signal. In all simulation examples, i.i.d. complex circular Gaussian receiver noise with an SNR of $10 \mathrm{~dB}$ is added to the measurements before applying the downsampling operation. As the focus is on target multipath, returns from the front wall are assumed to be properly suppressed. Also, direct returns from the back wall located at $6 \mathrm{~m}$ downrange are not 

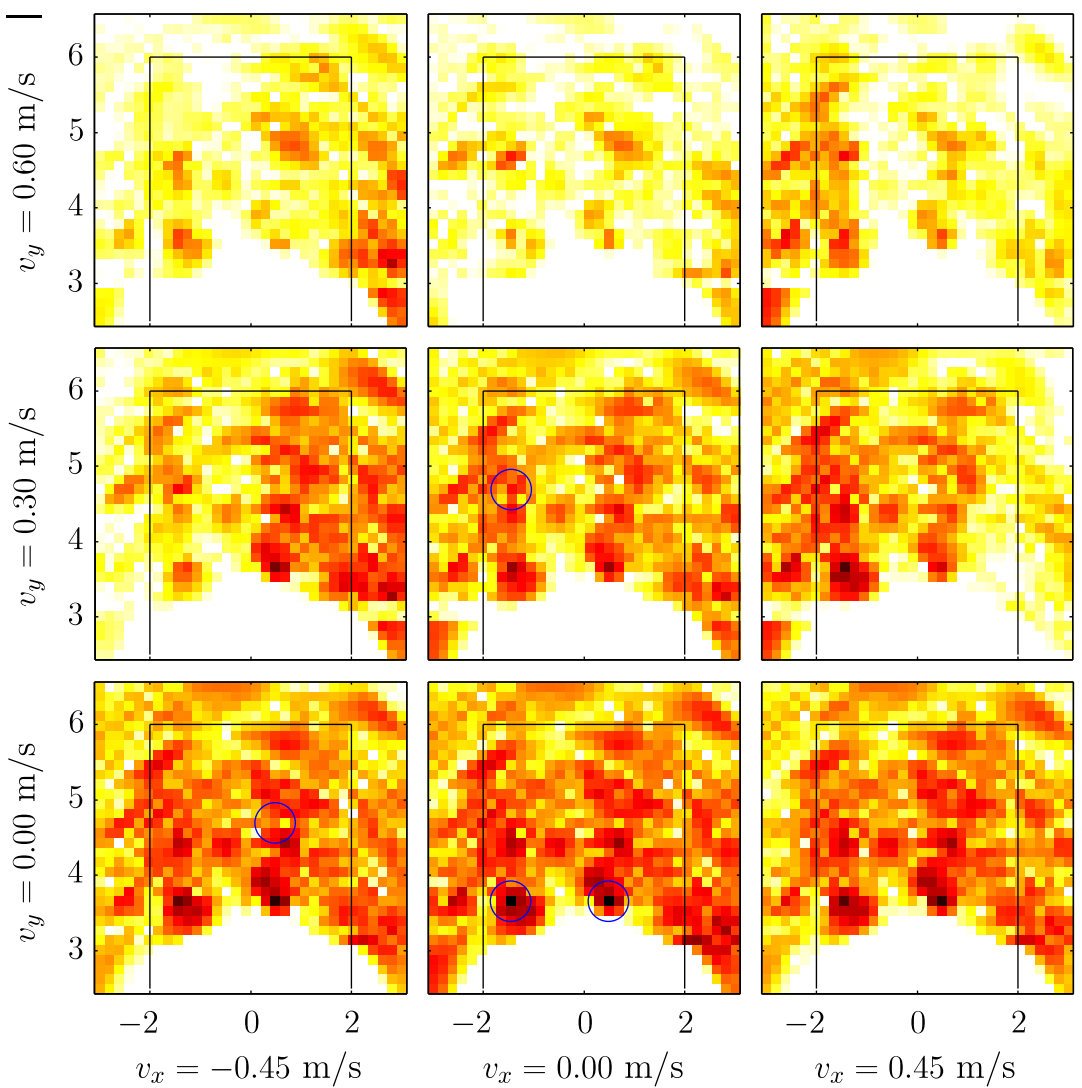

Figure 4.9. Conventional image formation result using full data.

considered. The imaged region extends $6 \mathrm{~m}$ in crossrange and $4 \mathrm{~m}$ in downrange and is centered around a point in the broadside direction of the array at $4 \mathrm{~m}$ downrange. The scene of interest is spatially discretized into an $P_{x} \times P_{y}=32 \times 32$ pixel grid. The target velocities are discretized on an $P_{v_{x}} \times P_{v_{y}}=5 \times 7$ crossrange by downrange grid, spanning target velocity components of $\pm 0.9 \mathrm{~m} / \mathrm{s}$.

Imaging Examples The simulated scene consists of two stationary targets residing at coordinates $(0.5,3.7) \mathrm{m}$ and $(-1.5,3.7) \mathrm{m}$ and two moving targets at $(0.5,4.7) \mathrm{m}$ and $(-1.5,4.7) \mathrm{m}$, respectively. The moving targets are assumed to be $8 \mathrm{~dB}$ weaker than the stationary targets and possess respective velocities $(-0.45,0) \mathrm{m} / \mathrm{s}$ and $(0,0.3) \mathrm{m} / \mathrm{s}$. At first, no returns from the room corners are considered. The four targets are visible via all $R=7$ possible paths.

The conventional beamforming results using full measurements are shown in Figure 4.9. The images are obtained by the velocity matched beamforming approach (4.5). Each sub-image represents the DSBF result matched to the corresponding target velocity as indicated on the bottom and left edge of the image. All images are shown on a 


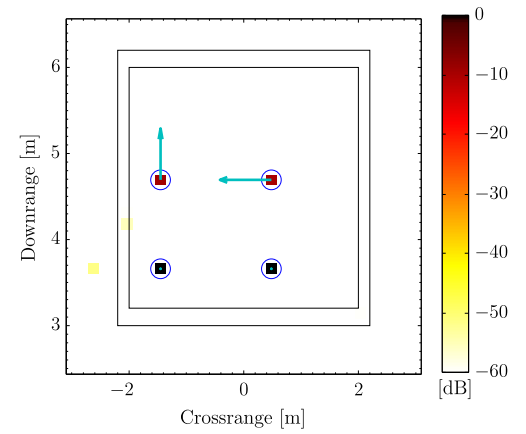

(a) GSCS using SparSA

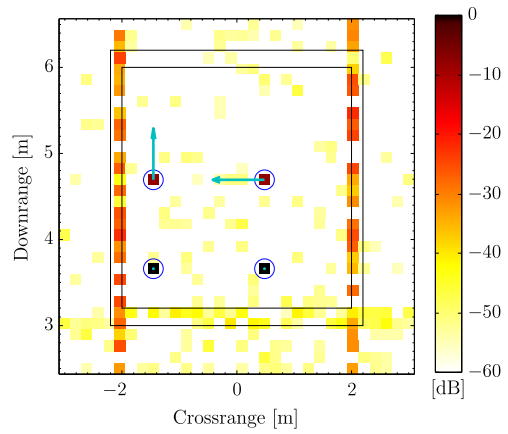

(b) GSCS using BOMP

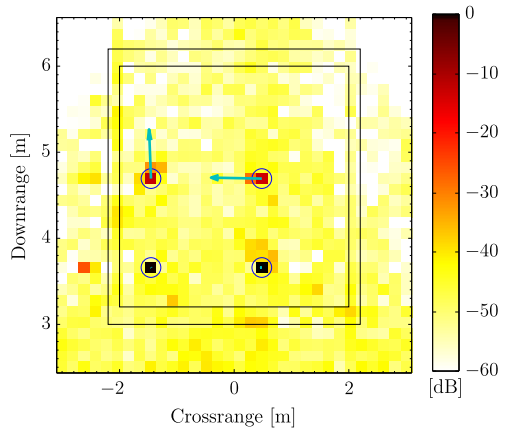

(c) Two-step approach

Figure 4.10. CS reconstruction using $7 \%$ of the measurements. Arrows indicate target velocities.

$40 \mathrm{~dB}$ scale. The image appears very cluttered due to the multipath responses. The targets cannot reliably be assigned to a velocity as there is a lot of leakage between the sub-images due to the limited velocity resolution of the beamforming approach.

Next, sparsity-based multipath exploitation results averaged over 20 Monte Carlo runs are presented in Figure 4.10. The downsampling parameters of (3.24) are set to $T_{\mathrm{d}}=$ 20, $M_{\mathrm{d}} N_{\mathrm{d}}=8$ and $K_{\mathrm{d}}=15$, performing linear measurements using a Gaussian random mixing matrix in fast time amounting to $7 \%$ of the full Nyquist sampled measurements. Three different algorithms are considered and compared: GSCS with joint location and velocity estimation using a) an optimization-based approach (SparSA) and b) a greedy approach (BOMP); c) the two-step approach with SparSA in the location reconstruction and least-squares method for velocity estimation. The regularization parameter in the SparSA reconstruction is set to $\lambda=\lambda_{\text {norm }}\left\|(\Phi \tilde{\Psi})^{H} \overline{\boldsymbol{z}}\right\|_{\infty}$, with $\lambda_{\text {norm }}=$ 0.1. The sparsity level for the BOMP is set 20 nonzero groups overestimating the true sparsity level. An accumulated image is shown, where the magnitudes are accumulated over velocities for the group sparse methods and over the slow time for the two-step approach. The velocity estimates for the four strongest targets are indicated using arrows. The group sparse reconstruction using SparSA in Figure 4.10a features perfect reconstruction of the target positions and velocities. The ghost targets have been suppressed and only a few very faint clutter pixels remain. Greedy reconstruction using the BOMP successfully finds the targets and their velocities, however, strong clutter along the side walls is present, see Figure 4.10b. The result of the two-step approach in Figure 4.10c generally shows larger background noise and lower SCR. The four targets and the corresponding velocities are reconstructed nonetheless.

To evaluate the numerical complexity, the average runtime of the following three algorithms is compared: joint estimation using SparSA, joint estimation using BOMP, 


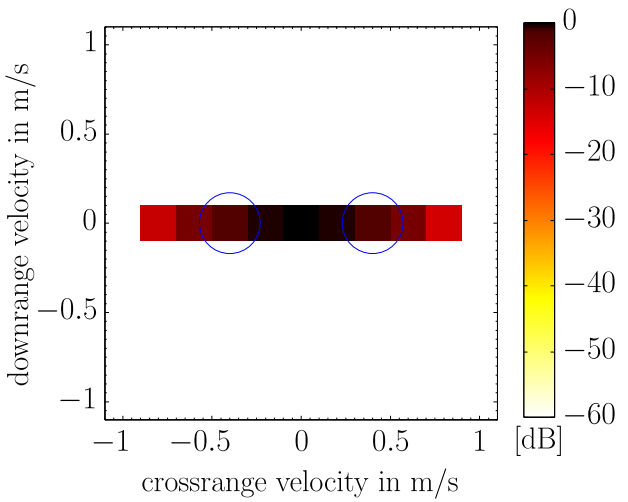

(a) Direct path only $(R=1)$

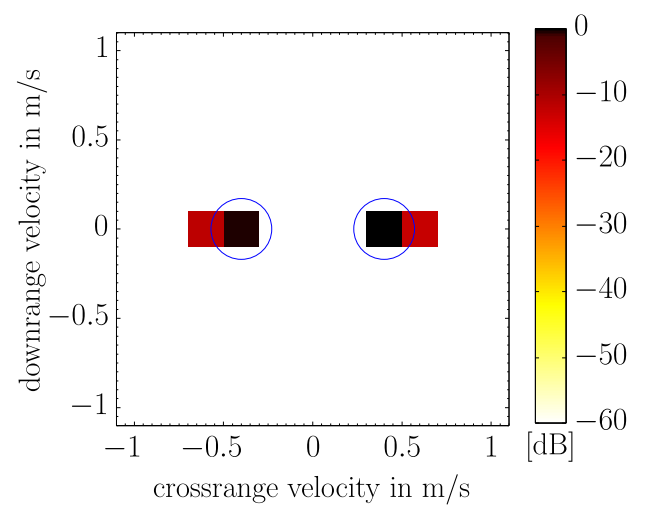

(c) One $2^{\text {nd }}$ order path $(R=6)$

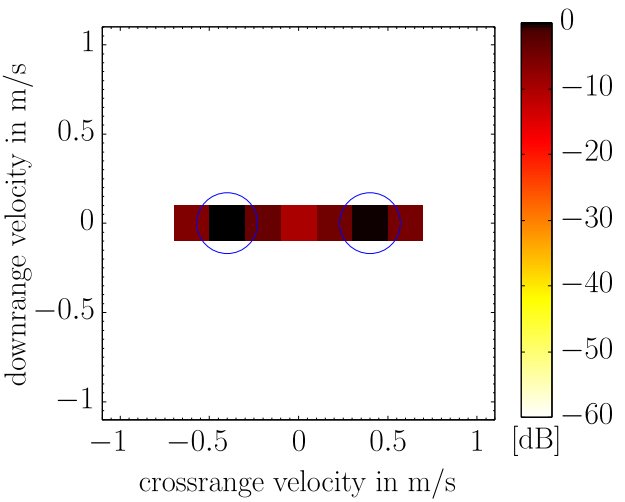

(b) $1^{\text {st }}$ order paths only $(R=5)$

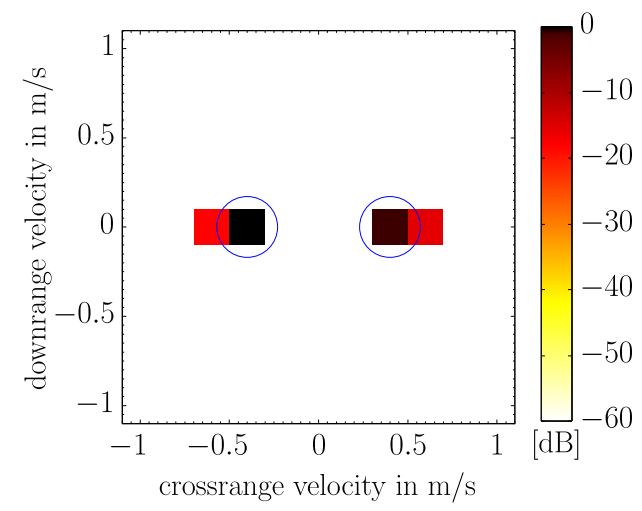

(d) All paths $(R=7)$

Figure 4.11. CS reconstruction results for various amounts of multipath.

and the two-step approach with SparSA employed in the first step. A single core of a 2.8 GHz CPU was used for the calculations. The average runtime of the joint estimation using SparSA was 170.3 min, while those for joint estimation using BOMP and the two-step approach were $11.1 \mathrm{~min}$ and $5 \mathrm{~min}$, respectively. Both BOMP-based joint estimation and the two-step method are one order of magnitude faster than the joint estimation using SparSA, with the two-step approach being the fastest due to its much reduced CS problem size.

Velocity estimation In the following set of simulations, the velocity estimation performance of the algorithms shall be investigated. First, it is shown that multipath returns can be exploited to improve the velocity resolution. The joint location and velocity estimation method (4.19) using SparSA is employed to resolve two targets with similar velocities. The targets are fixed at $4 \mathrm{~m}$ downrange in the broadside direction of the array, while the room and system parameters are kept the same as in the previous examples. Both targets reside in the same range/crossrange cell, but move in opposing crossrange directions. The $11 \times 11$ velocity grid covers range/crossrange 


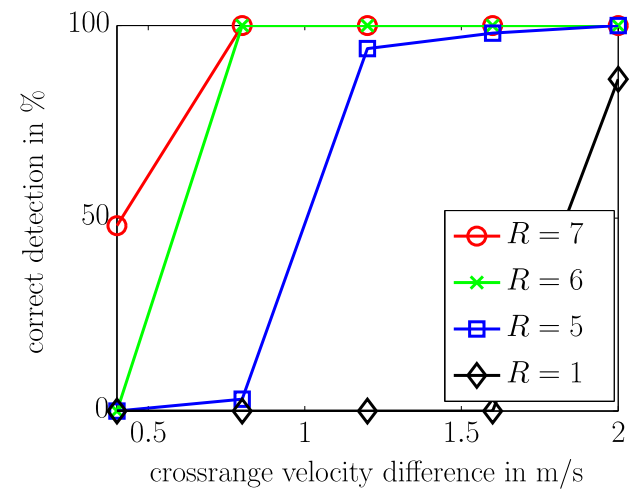

(a) CS using SparSA

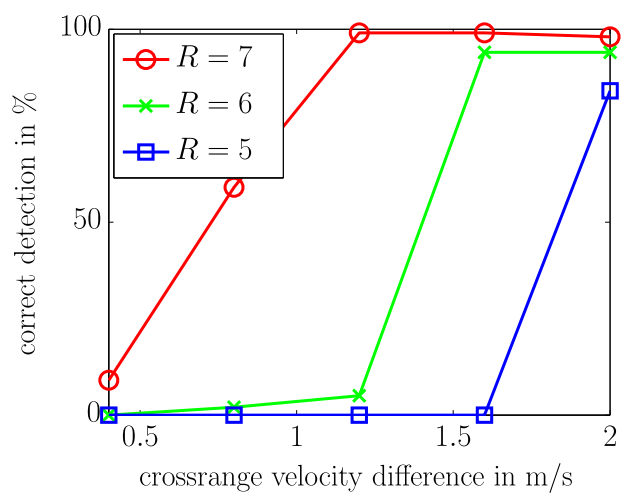

(c) Two-step method

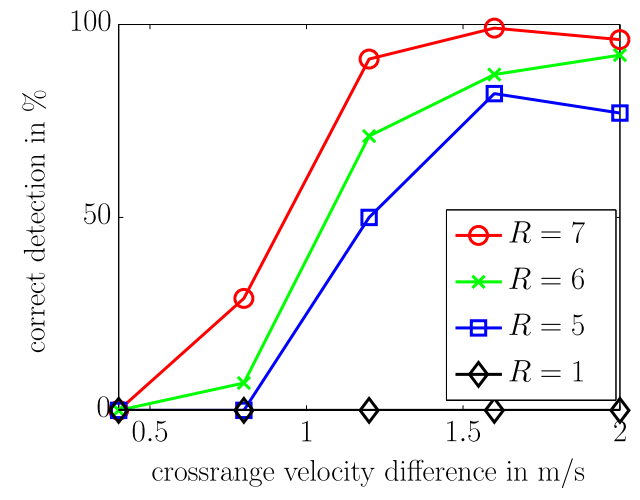

(b) CS using BOMP

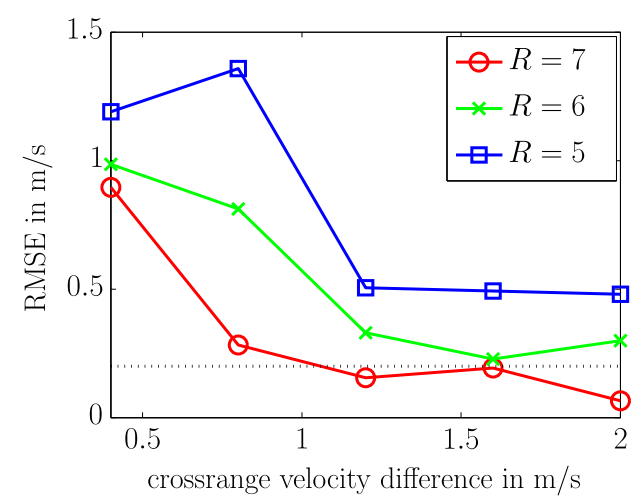

(d) Two-step method RMSE

Figure 4.12. Crossrange velocity resolution performance for various amounts of multipath and different reconstruction methods.

velocities between $\pm 1 \mathrm{~m} / \mathrm{s}$. The target velocities differ by only $0.8 \mathrm{~m} / \mathrm{s}$. In Figure 4.11 the results for various amounts of multipath are shown. Four cases are distinguished, namely, direct propagation path only $(R=1)$, direct and first order multipath returns only $(R=5)$, the former plus an additional second order multipath $(R=6)$ and all $R=7$ paths per target. It is evident that the velocity resolution capability improves with the incorporation of an increasing number of multipath returns. If only the direct path is available, the two moving targets cannot be resolved, as seen in Figure 4.11a. If all four first order multipath returns are included and exploited, the two targets are resolved, but surrounding clutter pixels may render the velocity estimation difficult, see Figure 4.11b. Finally, if 6 or 7 paths are available and exploited, the two moving targets are resolved with accurate velocity estimates, as evident in Figures 4.11c,d.

In order to quantify the velocity resolution performance for the proposed methods as a function of the number of multipath returns, the same setup as in the previous example is used. However, the velocity difference between the two targets is varied from $0.4 \mathrm{~m} / \mathrm{s}$ to $2 \mathrm{~m} / \mathrm{s}$ in steps of $0.4 \mathrm{~m} / \mathrm{s}$. The algorithms' ability to resolve the two targets 


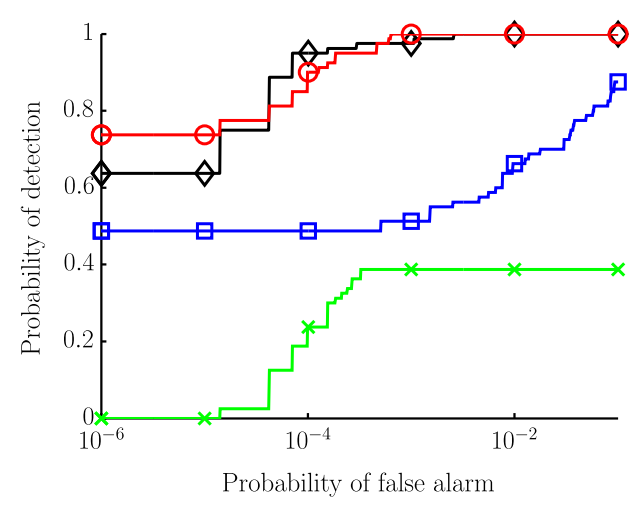

(a) Without corner returns

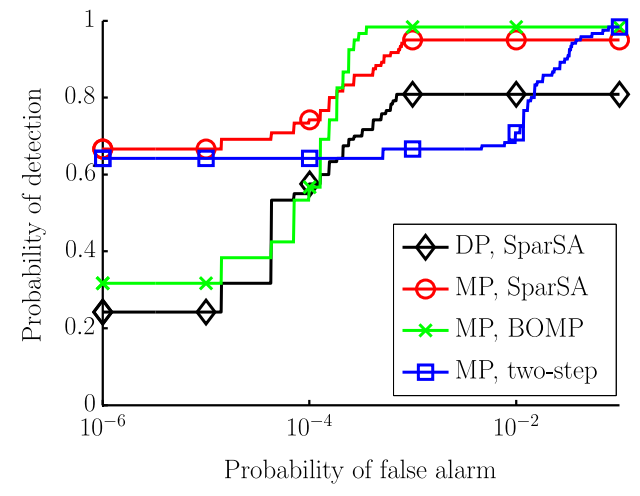

(b) Including corner returns

Figure 4.13. ROC curves for various CS reconstruction scenarios. The legend applies to both plots.

is assessed and the results are averaged over 100 Monte Carlo runs. A simplistic detection scheme serves as metric to provide a fair comparison for the examined cases. In the joint velocity estimation approaches, the two strongest pixels are selected. If they correspond to the true target velocities, the targets are deemed successfully resolved. To quantify the success of the two-step approach, first the RMSE of the velocity estimate is calculated. Secondly, the success of velocity resolution is evaluated, where an RMSE smaller than the velocity grid spacing, i.e., $0.2 \mathrm{~m} / \mathrm{s}$ means successful detection. Figure 4.12 summarizes the velocity estimation performance results. The velocity detection performance for reconstruction with SparSA, BOMP, and the two-step method is shown in Figures 4.12a-c, whereas Figure 4.12d depicts the RMSE of the velocity estimation of the two-step method along with the $0.2 \mathrm{~m} / \mathrm{s}$ threshold (dotted line). Observe that without multipath exploitation, none of the methods is able to resolve tightly-spaced velocities, while the resolution capabilities improve with increasing number of available multipath returns. This is also reflected by the estimation error plot of the two-step method as shown in Figure 4.12d: exploitation of more paths results in a lower RMSE. Furthermore, the resolution performance of the three methods scales with the computational complexity. While the two-step approach is computationally cheap, it performs rather poorly as compared to the other two, see Figure 4.12c. When using the joint reconstruction with BOMP, refer to Figure $4.12 \mathrm{~b}$, the performance improves, whereas the numerically demanding SparSA reconstruction in Figure 4.12a is clearly the best. From Figure 4.12 one can conclude that exploiting the information contained in the multipath returns improves scene reconstruction.

Receiver Operating Characteristic Curves The last set of simulations compares overall reconstruction performance by means of the Receiver Operating Characteristic 
(ROC) curve. The radar geometry and scene setup with four targets is the same as for the imaging examples. The ROC curves for the considered multi-target scene have been calculated in the following manner. Amplitude detection with a given threshold is used to form a binary image. A target pixel is considered to be correctly detected if the detected pixel coincides with the true target state or lies in one of the eight surrounding pixels. This must be fulfilled for both the location and the velocity. Several detected pixels within this neighborhood are treated as one. This is motivated by the fact successful detection of the target within its close vicinity is usually sufficient in TWRI. A false alarm event is defined as a pixel detected outside the neighborhood of any target which corresponds to an unwanted clutter or ghost pixel in the image. The simulation results are averaged over 20 Monte Carlo runs and the corresponding ROC curves are averaged on a common false alarm axis.

First, only target returns are considered in the received signal model. The undersampling parameters are selected as $T_{\mathrm{d}}=20, M_{\mathrm{d}} N_{\mathrm{d}}=2$, and $K_{\mathrm{d}}=15$. Various sparse reconstruction algorithms are considered for direct path (DP) and multipath (MP) propagation environments:

- DP, SparSA: Only the direct path is modeled, i.e., $R=1$ and conventional CS reconstruction using SparSA is employed. This corresponds to a multipath-free environment and can be seen as a benchmark scenario.

- MP, SparSA: GSCS-based multipath exploitation using SparSA is employed.

- MP, BOMP: GSCS-based multipath exploitation using BOMP is employed.

- MP, two-step: Two-step multipath exploitation is employed.

All $R=7$ paths are modeled for the latter three reconstruction approaches.

Figure 4.13a depicts the corresponding ROC curves. Considering the multipath scenario, reconstruction using the optimization-based approach ("MP, SparSA") performs best, followed by the two-step approach and the BOMP. Note that for the two-step approach only the target location performance is considered in the ROC while velocity estimation is neglected, which may explain the advantage over the BOMP. Joint reconstruction based on SparSA results in approximately equal ROC curves regardless if multipath is present or not. Hence, proper exploitation of multipath results in a performance comparable with the multipath-free case.

The above simulation is repeated with the returns from the two corners in the back of the room included in the received signal. The corner returns are modeled according 


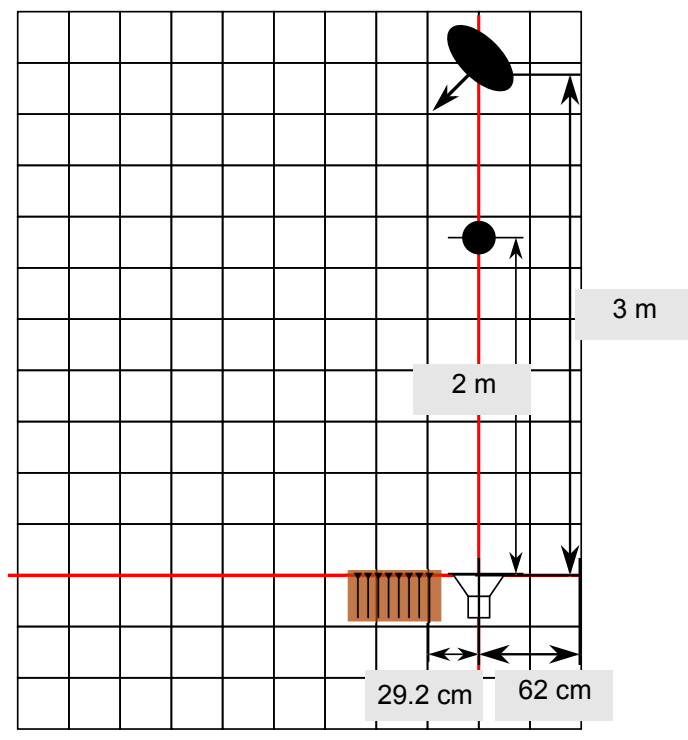

Figure 4.14. Scene setup of a human (ellipse) walking diagonally towards the radar. Smaller stationary object (circle) resides a lower downrange.

to (3.19) with $N_{\mathrm{c}}=2$ and $L_{u}=2 \mathrm{~m}, u=\{0,1\}$. The number of used transceiver pairs has been increased to $M_{\mathrm{d}} N_{\mathrm{d}}=8$ to accommodate for the reduced sparsity in the reconstruction. The resulting ROC curves are presented in Figure 4.13b. The locations of the corners are considered stationary targets when calculating the ROC. In this case, multipath is clearly a benefit rather than a nuisance. By exploiting the signal power contained in the target multipath, the detection performance can be improved in a scenario with strong stationary clutter. Both multipath exploitation algorithm using SparSA and BOMP, respectively, outperform the multipath-free reconstruction result. The two-step approach, however, seems to suffer significantly from the corner clutter. Note that due to the reduced sparsity of the scene, the two SparSA methods fail to reconstruct all targets leading to an ROC that saturates at $P_{D}<0$. This could be avoided by properly tuning the regularization parameter. However, $\lambda_{\text {norm }}$ was kept at the same level throughout the results to maintain comparability.

\section{Experimental Results}

Experimental results are presented for a wideband real aperture pulse-Doppler radar with $M=1$ transmitter and a uniform linear array with $N=8$ receivers. The data has been recorded at the Radar Imaging Lab, Villanova University, in a semicontrolled lab setup. Refer to Figure 4.14 for an illustration of the scene setup. The transmit waveform is a modulated Gaussian pulse, covering the frequency range of 1.5 to $4.5 \mathrm{GHz}$. Eat each receiver, 768 fast time samples have been recorded at a 


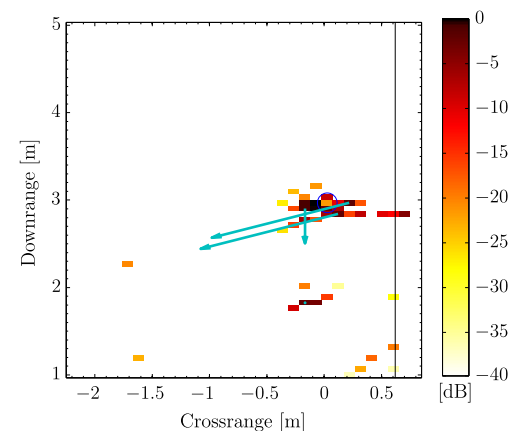

(a) GSCS using SparSA

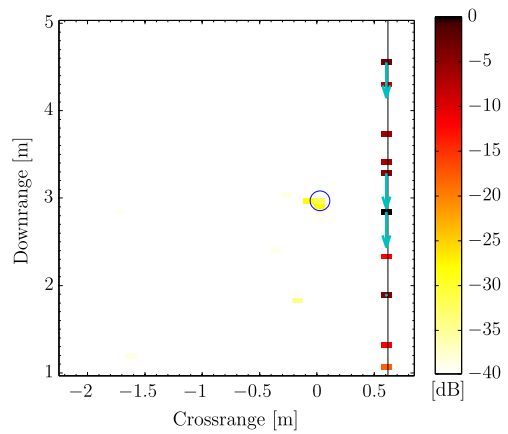

(b) GSCS using BOMP

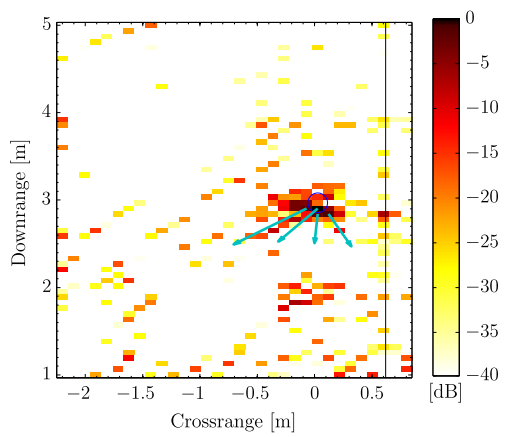

(c) Two-step approach

Figure 4.15. CS reconstruction of the walking human using $20 \%$ of the measurements. Arrows indicate target velocities.

sampling rate $f_{s}=7.68 \mathrm{GHz}$. Early and late returns have been gated out to clean the data, resulting in $T=153$ samples. The transmitter was placed $62 \mathrm{~cm}$ away from a side wall and the receive array (element spacing $6 \mathrm{~cm}$ ) was placed on the other side of the transmitter at a distance (to the first element) of $29.2 \mathrm{~cm}$ on the same baseline. No front wall was present in the scene. This is because, if present, the wall EM scatterings can be either mitigated [AA12b] or gated out in wideband bistatic pulsed radar measurements [QAA13]. A total of $R=4$ possible propagation paths are expected, namely, the direct path, two first order and one second order multipath via the side wall. The scene of interest is spatially discretized into a $P_{x} \times P_{y}=32 \times 64$ pixel grid. The target velocities are discretized on an $P_{v_{x}} \times P_{v_{y}}=5 \times 7$ grid spanning target velocity components of $\pm 0.6 \mathrm{~m} / \mathrm{s}$. A scenario with a human walking diagonally towards the radar was recorded. All of the above mentioned propagation paths are expected to be observed for the human.

The three CS-based multipath exploitation methods are employed and the imaging results are shown in Figure 4.15 along with the velocities of the four strongest targets. The downsampling parameters of (3.24) are set to $T_{\mathrm{d}}=50, N_{\mathrm{d}}=5$ and $K_{\mathrm{d}}=15$ amounting to $20 \%$ of the full Nyquist measurements. To account for the higher amount of clutter and noise in the experimental data, the regularization parameter is set to $\lambda_{\text {norm }}=0.3$. The sparsity level for BOMP is kept at 20. The joint location and velocity estimation method using SparSA, see Figure 4.15a, and the two-step approach, c.f. Figure $4.15 \mathrm{c}$, are able to recover the moving human at the correct location. The directions of the estimated velocity vectors is generally consistent with the ground truth. However, the exact velocity estimates differ considerably. This may be attributed to the complex nature of torso and limb movements. Additional clutter at $2 \mathrm{~m}$ downrange can be attributed to some stationary objects present in the lab. Furthermore, the imaging result of the two-step approach shows inferior SCR. Note that the reconstruction 
using BOMP in Figure 4.15b fails completely. Greedy methods are more susceptible to dictionary mismatch, i.e., clutter in the radar sense, which may lead to the observed failure.

\subsection{Distributed Radar}

For ground-based operation, deployment of a network of multistatic radar units, each with a limited number of transmitters and receivers, can provide an effective and agile alternative to vehicle-mounted systems. Measurements of several such units, that may or may not cooperate, are acquired. The reconstruction should be performed jointly on the whole data set to utilize the additional spatial diversity of the distributed radar configuration. The contributions of this section were reported in [LAAZ15c, LAAZ15d].

\subsubsection{Multiple Radar Unit Model}

Before turning to the reconstruction problem, a multiple radar unit model is developed. The model is built on the single multistatic pulsed radar units as described in Chapter 3. Various possibilities of deploying several of these units are described and analyzed. Instead of a single radar, $S$ radar units are deployed around the scene of interest. Depending on the capabilities of the units, two operating principles are considered. In independent operation, each unit transmits and receives without cooperation with other units. Interference should be avoided by separating transmission in time or frequency bands. This scheme results in $S$ measurement vectors, one from each unit. In cooperative operation, each transmitted pulse is assumed to be received simultaneously by all receivers from all units. Hence, the total number of measurements is increased $S^{2}$-fold as compared to the single radar unit case. Furthermore, two cases of radar unit placement are distinguished, namely, the co-located and the distributed configurations. In the co-located configuration, the modules are positioned next to each other at the same standoff distance from the front wall, emulating a multistatic radar system with much longer transmit and receive array apertures. The resulting system views the scene approximately from the same angle, thereby permitting the RCS changes to be neglected and all of the measurements to be coherently combined. Note that this case is equivalent to the single unit model where the transmit and receive arrays from all units are combined. The distributed case offers the additional flexibility of deploying the radar modules widely separated with arbitrary standoff distances either along the front wall or surrounding the scene of interest. As such, the aspect and/or bistatic angles 
and, in turn, the RCS changes across the units. Therefore, the acquired measurements cannot be combined coherently across the radar units.

In the sequel, the signal models for the four cases are developed. For each configuration, the measurement vectors $\left\{\overline{\boldsymbol{z}}_{s^{\mathrm{Tx}} s^{\mathrm{Rx}}} ; s^{\mathrm{Tx}}, s^{\mathrm{Rx}}=0, \ldots, S-1\right\}$ are obtained, where $s^{\mathrm{Tx}}$ and $s^{\mathrm{Rx}}$ are the indices of the transmitting and receiving modules, respectively. Likewise, the dictionaries $\tilde{\boldsymbol{\Psi}}_{s^{\mathrm{Tx}}{ }_{s}^{\mathrm{Rx}}}$ are indexed by the transmitting and receiving modules. Finally, the spatial downsampling operation for the transmitting module $s^{\mathrm{Tx}}$ is denoted as $\boldsymbol{\Phi}_{s^{\mathrm{Tx}} \mathrm{T}}^{\mathrm{Tx}}$, which indicates the active transmitters. At the receiving side, $\boldsymbol{\Phi}_{s^{\mathrm{Rx}}}^{\mathrm{Rx}}$ represents the downsampling matrix for the receiving module $s^{\mathrm{Rx}}$, which includes not only the spatial downsampling of the $N$ receivers but also the linear mixing scheme for downsampling in the time domain. An additive noise vector $\tilde{\boldsymbol{n}}$ for the full received signal is also considered. Using the above notation, the signal model in the independent and colocated case can be expressed as

$$
\left[\begin{array}{c}
\overline{\boldsymbol{z}}_{0} 0 \\
\overline{\boldsymbol{z}}_{1} 1 \\
\vdots \\
\overline{\boldsymbol{z}}_{S-1} S_{-1}
\end{array}\right]=\left(\begin{array}{c}
\boldsymbol{\Phi}_{0}^{\mathrm{Tx}} \boldsymbol{\Phi}_{0}^{\mathrm{Rx}} \tilde{\boldsymbol{\Psi}}_{0} \\
\boldsymbol{\Phi}_{1}^{\mathrm{Tx}} \boldsymbol{\Phi}_{1}^{\mathrm{Rx}} \tilde{\boldsymbol{\Psi}}_{1} 1 \\
\vdots \\
\boldsymbol{\Phi}_{S-1}^{\mathrm{Tx}} \boldsymbol{\Phi}_{S-1}^{\mathrm{Rx}} \tilde{\boldsymbol{\Psi}}_{S-1}
\end{array}\right) \tilde{\boldsymbol{\sigma}}+\tilde{\boldsymbol{n}}
$$

The signal model in the cooperative and co-located case can be written as

$$
\left[\begin{array}{c}
\overline{\boldsymbol{z}}_{0} 0 \\
\overline{\boldsymbol{z}}_{0} 1 \\
\vdots \\
\overline{\boldsymbol{z}}_{0 S-1} \\
\vdots \\
\overline{\boldsymbol{z}}_{S-1} S_{-1}
\end{array}\right]=\left(\begin{array}{c}
\boldsymbol{\Phi}_{0}^{\mathrm{Tx}} \boldsymbol{\Phi}_{0}^{\mathrm{Rx}} \tilde{\boldsymbol{\Psi}}_{0} 0 \\
\boldsymbol{\Phi}_{0}^{\mathrm{Tx}} \boldsymbol{\Phi}_{1}^{\mathrm{Rx}} \tilde{\boldsymbol{\Psi}}_{0} 1 \\
\vdots \\
\boldsymbol{\Phi}_{0}^{\mathrm{Tx}} \boldsymbol{\Phi}_{S-1}^{\mathrm{Rx}} \tilde{\boldsymbol{\Psi}}_{0}{ }_{S-1} \\
\vdots \\
\boldsymbol{\Phi}_{S-1}^{\mathrm{Tx}} \boldsymbol{\Phi}_{S-1}^{\mathrm{Rx}} \tilde{\boldsymbol{\Psi}}_{S-1}{ }_{S-1}
\end{array}\right) \tilde{\boldsymbol{\sigma}}+\tilde{\boldsymbol{n}} .
$$

Note that in the co-located case, a common reflectivity vector $\tilde{\boldsymbol{\sigma}}$ is sufficient for all combinations of the various modules, as the target RCS is assumed to be the same in this case.

In contrast to the co-located configuration, a separate reflectivity vector $\tilde{\boldsymbol{\sigma}}_{s^{\mathrm{Tx}} s^{\mathrm{Rx}}}$ for each combination of $s^{\mathrm{Tx}}$ and $s^{\mathrm{Rx}}$ is introduced in the distributed case. This accounts for the RCS change that is inherent in a distributed aperture scenario. Hence, for the independent and distributed configuration, one obtains

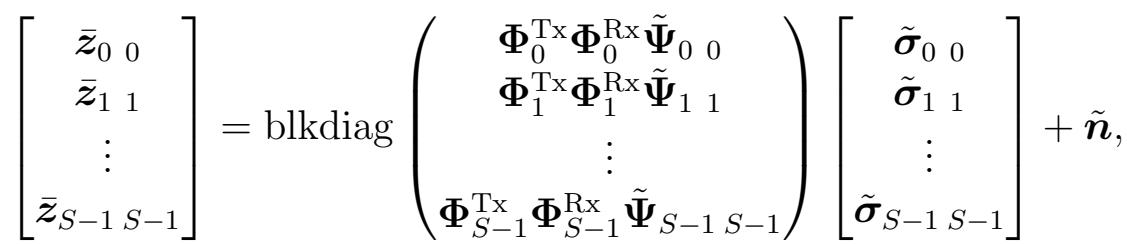


where blkdiag $(\cdot)$ denotes the block diagonal matrix operation. In the cooperative and distributed case, the model is described as

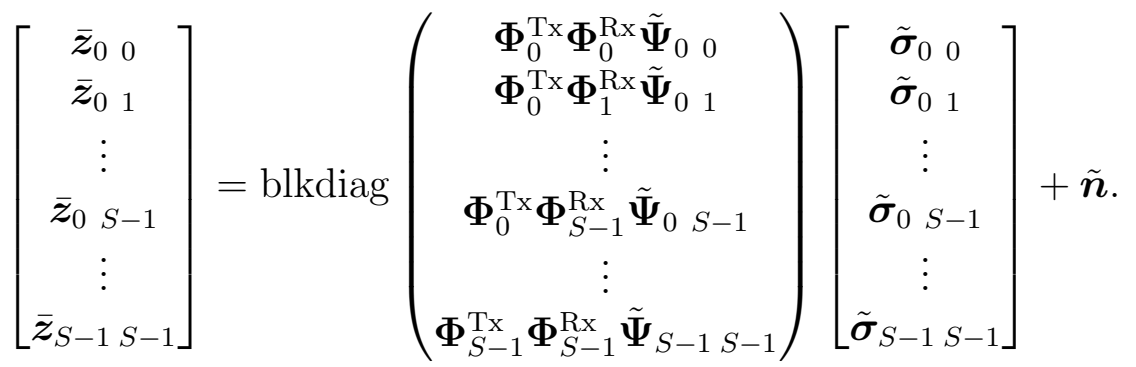

Note that the number of unknowns has increased $S$-fold or $S^{2}$-fold, respectively, as compared to the co-located case. However, this increase can be compensated by exploiting the structure contained in the combined vector of unknowns. Similar to the multipath exploitation case, each transmit-receive module pair observes the same scene of interest. Hence, due to the point target assumption, the locations of the targets are invariant. This results in a group sparse structure, and all $\tilde{\boldsymbol{\sigma}}_{s^{\mathrm{Tx}} s^{\mathrm{Rx}}}$ vectors share a common support or sparsity pattern which is detailed in Section 4.5.3.

Note that independent operation is a special case of cooperative operation. By deleting the appropriate lines in the cooperative model (4.27) and (4.29), one can obtain the independent operation models (4.26) and (4.28). Hence, in the sequel, only the general cooperative case is considered as it includes independent operation.

\subsubsection{Dictionary Analysis}

In order to compare the co-located and distributed cases, the two dictionaries in the respective models (4.27) and (4.29) are analyzed in more detail. In both cases, the

dictionary matrices are composed of the same blocks $\boldsymbol{\Phi}_{s^{\mathrm{Tx}}}^{\mathrm{Tx}} \boldsymbol{\Phi}_{s^{\mathrm{Rx}}}^{\mathrm{Rx}} \tilde{\boldsymbol{\Psi}}_{s^{\mathrm{Tx}} s^{\mathrm{Rx}}}$, where $s^{\mathrm{Tx}}, s^{\mathrm{Rx}}=$ $0, \ldots, S-1$. For notational convenience, a linear index for the blocks $b=0, \ldots, B-1=$ $S^{2}$ is used and the dictionary blocks are defined as

$$
\boldsymbol{A}^{(b)}=\boldsymbol{\Phi}_{s^{\mathrm{Tx}}}^{\mathrm{Tx}} \boldsymbol{\Phi}_{s^{\mathrm{Rx}}}^{\mathrm{Rx}} \tilde{\boldsymbol{\Psi}}_{s^{\mathrm{Tx}} s^{\mathrm{Rx}}}, \quad s^{\mathrm{Tx}}=\left\lfloor\frac{b}{S}\right\rfloor, \quad s^{\mathrm{Rx}}=b \bmod S, \quad b=0, \ldots, B-1 .
$$

Hence, the dictionary in the co-located model (4.27) can be expressed as

$$
\boldsymbol{A}_{\mathrm{co-loc}}=\left[\left(\boldsymbol{A}^{(0)}\right)^{T}, \ldots,\left(\boldsymbol{A}^{(B-1)}\right)^{T}\right]^{T}
$$

and the one for the distributed model (4.29) as

$$
\boldsymbol{A}_{\mathrm{distr}}=\operatorname{blkdiag}\left(\boldsymbol{A}^{(0)}, \ldots, \boldsymbol{A}^{(B-1)}\right) .
$$


Without loss of generality, in the sequel, normalized columns are assumed for the sub-matrices $\boldsymbol{A}^{(b)}$ in (4.31) and (4.32), i.e., $\left\|\boldsymbol{a}_{i}^{(b)}\right\|_{2}=1, \forall i, \forall b=0, \ldots, B-1$. From (2.4) follows that the coherence values of the individual sub-matrices are $\mu\left(\boldsymbol{A}^{(b)}\right)=$ $\max _{i, j \neq i}\left|\left(\boldsymbol{a}_{i}^{(b)}\right)^{H} \boldsymbol{a}_{j}^{(b)}\right|=\mu_{b}, b=0, \ldots, B-1$. In the co-located case, the coherence can be calculated as

$$
\begin{aligned}
\mu\left(\boldsymbol{A}_{\text {co-loc }}\right) & =\max _{i, j \neq i} \frac{\left|\left(\boldsymbol{a}_{i}^{(0)}\right)^{H} \boldsymbol{a}_{j}^{(0)}+\cdots+\left(\boldsymbol{a}_{i}^{(B-1)}\right)^{H} \boldsymbol{a}_{j}^{(B-1)}\right|}{\left\|\left(\boldsymbol{a}_{i}^{(0)}\right)^{T}, \ldots,\left(\boldsymbol{a}_{i}^{(B-1)}\right)^{T}\right\|_{2}\left\|\left(\boldsymbol{a}_{j}^{(0)}\right)^{T}, \ldots,\left(\boldsymbol{a}_{j}^{(B-1)}\right)^{T}\right\|_{2}} \\
& \leq \frac{\max _{i, j \neq i}\left|\left(\boldsymbol{a}_{i}^{(0)}\right)^{H} \boldsymbol{a}_{j}^{(0)}\right|+\cdots+\max _{i, j \neq i}\left|\left(\boldsymbol{a}_{i}^{(B-1)}\right)^{H} \boldsymbol{a}_{j}^{(B-1)}\right|^{(B)} \sqrt{B}}{\sqrt{B}} \\
& =\frac{1}{B} \sum_{b=0}^{B-1} \mu_{b} .
\end{aligned}
$$

In essence, the overall dictionary coherence is less than or equal to the arithmetic mean of the individual coherences of the sub-matrices.

For the distributed case, the coherence is given by

$$
\mu\left(\boldsymbol{A}_{\text {distr }}\right)=\max _{b} \max _{i, j \neq i} \frac{\left|\left(\boldsymbol{a}_{i}^{(b)}\right)^{H} \boldsymbol{a}_{j}^{(b)}\right|}{\left\|\left(\boldsymbol{a}_{i}^{(b)}\right)\right\|_{2}\left\|\left(\boldsymbol{a}_{j}^{(b)}\right)\right\|_{2}}=\max _{b} \mu_{b},
$$

which implies that the overall dictionary coherence is the maximum of the individual coherences of the sub-matrices. Clearly, $\mu\left(\boldsymbol{A}_{\text {distr }}\right) \geq \mu\left(\boldsymbol{A}_{\text {co-loc }}\right)$. Furthermore, the distributed configuration suffers from the $B$-fold increase in the ambient dimension and in the number of non-zero elements in the solution which puts it at a disadvantage compared to the co-located case. However, the underlying structure of the solution of equation (4.29) has not been taken into account, yet. Since all sub-vectors $\tilde{\boldsymbol{\sigma}}_{s^{\mathrm{Tx}}{ }_{s}^{\mathrm{Rx}}}$ in (4.29) share the same sparsity pattern, rather than calculating the coherence of $\boldsymbol{A}_{\text {distr }}$, the block-coherence of the dictionary as defined in (2.5) is the appropriate metric. The block-coherence takes the advantage of exploiting block sparsity during reconstruction into account.

The definition of the block-coherence (2.5) assumes that the group sparse dictionary atoms reside in adjacent columns. However, in the problem at hand, a group consist of all columns associated with a certain target for any radar unit pair. As these columns do not lie adjacent to each other in $\boldsymbol{A}_{\text {distr }}$, the dictionary needs to be rearranged. The permuted dictionary is defined as

$$
\tilde{\boldsymbol{A}}_{\text {distr }}=\left[\operatorname{blkdiag}\left(\boldsymbol{a}_{0}^{(0)}, \ldots, \boldsymbol{a}_{0}^{(B-1)}\right), \ldots, \operatorname{blkdiag}\left(\boldsymbol{a}_{P-1}^{(0)}, \ldots, \boldsymbol{a}_{P-1}^{(B-1)}\right)\right],
$$


which obeys the block-structure as assumed in (2.5). This yields for the block-coherence in the distributed configuration

$$
\begin{aligned}
\mu_{\mathrm{B}}\left(\tilde{\boldsymbol{A}}_{\mathrm{distr}}\right) & =\max _{i, j \neq i} \frac{1}{B} \rho\left(\operatorname{blkdiag}\left(\boldsymbol{a}_{i}^{(0)}, \ldots, \boldsymbol{a}_{i}^{(B-1)}\right)^{H} \operatorname{blkdiag}\left(\boldsymbol{a}_{j}^{(0)}, \ldots, \boldsymbol{a}_{j}^{(B-1)}\right)\right) \\
& =\max _{i, j \neq i} \frac{1}{B} \rho\left(\operatorname{diag}\left(\left(\boldsymbol{a}_{i}^{(0)}\right)^{H} \boldsymbol{a}_{j}^{(0)}, \ldots,\left(\boldsymbol{a}_{i}^{(B-1)}\right)^{H} \boldsymbol{a}_{j}^{(B-1)}\right)\right) \\
& =\max _{i, j \neq i} \frac{1}{B} \max \left(\left|\left(\boldsymbol{a}_{i}^{(0)}\right)^{H} \boldsymbol{a}_{j}^{(0)}\right|, \ldots,\left|\left(\boldsymbol{a}_{i}^{(B-1)}\right)^{H} \boldsymbol{a}_{j}^{(B-1)}\right|\right) \\
& =\frac{1}{B} \max _{b} \mu_{b}=\frac{1}{B} \mu\left(\boldsymbol{A}_{\text {distr }}\right),
\end{aligned}
$$

which is an improvement by a factor of $B$ as compared to the conventional coherence.

Having derived the coherence values in (4.33), (4.34) and (4.36), the reconstruction guarantees for the corresponding cases shall be compared. For the considered configurations, the following upper bounds can be established for the number of targets (or sparsity of the scene),

$$
\begin{aligned}
\beta_{\text {co-loc }} & <\frac{1}{2}\left(\mu\left(\boldsymbol{A}_{\text {co-loc }}\right)^{-1}+1\right) \\
\beta_{\text {distr,nonblk }} & <\frac{1}{2 B}\left(\mu\left(\boldsymbol{A}_{\text {distr }}\right)^{-1}+1\right) \\
\beta_{\text {distr,blk }} & <\frac{1}{2 B}\left(\mu_{\mathrm{B}}\left(\tilde{\boldsymbol{A}}_{\text {distr }}\right)^{-1}+B\right)=\frac{1}{2}\left(\mu\left(\boldsymbol{A}_{\text {distr }}\right)^{-1}+1\right) .
\end{aligned}
$$

It is clear that without considering the block structure for the distributed case, the maximum number of targets is reduced by approximately a factor of $B$ from the colocated to the distributed configuration. However, when exploiting the group structure, the maximum number of targets $\beta_{\text {distr,blk }}$ is of the same order as for the co-located case. A performance degradation is expected as the coherence number $\mu\left(\boldsymbol{A}_{\text {distr }}\right)$ is usually larger than $\mu\left(\boldsymbol{A}_{\text {co-loc }}\right)$ as shown in Equations (4.33) and (4.34). Further, note that equations (4.37)-(4.39) do not hold exactly in the presence of noise. However, they are expected to give a reasonable indication of the reconstruction performance. This will be supported by simulation in Section 4.5.4.

\subsubsection{Joint Group Sparse Reconstruction}

For scene reconstruction, the methods from Sections 4.3 and 4.4 are adapted. It is assumed that the measurements from all units are available at a common data processing center, also referred to as centralized reconstruction. The signal models (4.27) and (4.29), corresponding to the co-located and distributed configurations, can be expressed using a common structure. The respective left-hand and right-hand sides of the 
models can be stacked into single vectors $\breve{\boldsymbol{z}}$ and $\breve{\boldsymbol{\sigma}}$, respectively, whereas the dictionary matrices are combined to $\breve{\boldsymbol{A}}$. Hence, the same overall measurement model is obtained for co-located and distributed units

$$
\breve{\boldsymbol{z}}=\breve{\boldsymbol{A}} \breve{\boldsymbol{\sigma}}+\tilde{\boldsymbol{n}} .
$$

The differences lie in the dimensions of the reflectivity vector $\breve{\boldsymbol{\sigma}}$ and the dictionary $\breve{\boldsymbol{A}}$ and the size of the groups in the group sparse reconstruction. Note that the underlying structure due to the multipath model with $R$ propagation paths is still present. A comparison of the relevant properties is provided in Table 4.1. As the measurements from the various modules are coherently combined in the co-located case, the number of unknowns and the group size does not change as compared to the single module case. In fact, the multi-module system is equivalent to a single radar unit with larger arrays. Conversely, the reflectivity vectors in the distributed case are different for each module pair, hence, the number of unknowns increases by a factor of $S^{2}$. In order to exploit the inherent sparsity structure, the group size is also increased by a factor of $S^{2}$. As discussed in the dictionary analysis, the larger group size at least partly offsets the increase in the number of unknowns.

For the multiple module case, we can employ the same mixed-norm reconstruction as in (4.19), resulting in

$$
\hat{\boldsymbol{\sigma}}=\arg \min _{\breve{\boldsymbol{\sigma}}} \frac{1}{2}\|\breve{\boldsymbol{z}}-\breve{\boldsymbol{A}} \breve{\boldsymbol{\sigma}}\|_{2}^{2}+\lambda\|\breve{\boldsymbol{\sigma}}\|_{2,1}
$$

Note that the regularization term $\|\breve{\boldsymbol{\sigma}}\|_{2,1}$ depends on the number and structure of the groups. In the co-located case, a group covers the reflectivities associated with all possible propagation paths for a specific target. In the distributed case, the groups are extended to include the available transmit-receive module pairs. Thus, a group contains the target reflectivities for all possible paths and module pairs amounting to a group size of $R S^{2}$. Again, the convex problem (4.41) can be reconstructed by SparSA or similar methods as described earlier in this thesis.

Note that the distributed radar approach lends itself to another extension. If the targets in the scene are frequency dependent, the measurements can be split into various frequency bands wherein the target RCS is constant. Hence, a further layer of grouping is introduced by splitting the reflectivity vectors into different frequency bands and grouping the corresponding frequency. This, in turn, increases the number of unknowns as well as the group size by the number of considered frequency bands. 
Table 4.1. Summary of key parameters in group sparse reconstruction

\begin{tabular}{l|c|c|c} 
& Single Module & Co-Located & Distributed \\
\hline $\begin{array}{l}\text { No. of transmit-receive } \\
\text { module pairs }\end{array}$ & 1 & $S^{2}$ & $S^{2}$ \\
$\begin{array}{l}\text { No. of unknown reflectivity } \\
\text { vectors or sub-images }\end{array}$ & 1 & 1 & $S^{2}$ \\
Group size & $R$ & $R$ & $R S^{2}$
\end{tabular}

\subsubsection{Simulation Results}

Simulations were performed for a simple rectangular room enclosed by four homogeneous walls. The room and wall parameters are the same as in Section 4.4. Again, the region of interest is discretized into $P_{x} \times P_{y}=64 \times 64$ pixels. Two multistatic radar units are placed outside the room in various configurations, as detailed later. Each of the $S=2$ multistatic radar units has a uniform linear array with $N=3$ receivers and an inter-element spacing of $10 \mathrm{~cm}$. The central element also acts as a transmitter, i.e., $M=1$. When one unit is transmitting, both units simultaneously record the returns with all of their receivers. All measurements are finally assumed to be available at a single data processing center where the scene recovery is carried out. The transmitter sends the same modulated Gaussian pulse as used in the previous simulation section and, again, $T=150$ fast time samples are collected at each receiver. The multipath environment is also assumed unchanged. Thus, in total, there are $R=7$ paths that are considered in the received signal corresponding to each transmit-receive pair. In all simulations, i.i.d. complex circular Gaussian noise with $20 \mathrm{~dB}$ SNR is added to the simulated measurements. In order to keep the focus on co-located and distributed radar configuration, the scenario is restricted to stationary targets. Thus, it is sufficient to consider a single pulse in the slow time domain, i.e., $K=1$.

Scenario A Eight point targets in a cross-shaped arrangement are simulated in the room, as illustrated in Figure 4.16. The reflectivity of the inner four targets is $6 \mathrm{~dB}$ lower as compared to the outer targets. In the distributed radar configuration, the two modules are located on opposing sides of the room, as shown in Figure 4.16a, whereas both modules are placed next to each other facing the front wall in the co-located case, see Figure 4.16b. The scene is reconstructed almost perfectly for both configurations, refer to Figure 4.17. In the given example, the reconstruction quality of the co-located modules is slightly better than for the distributed case. 


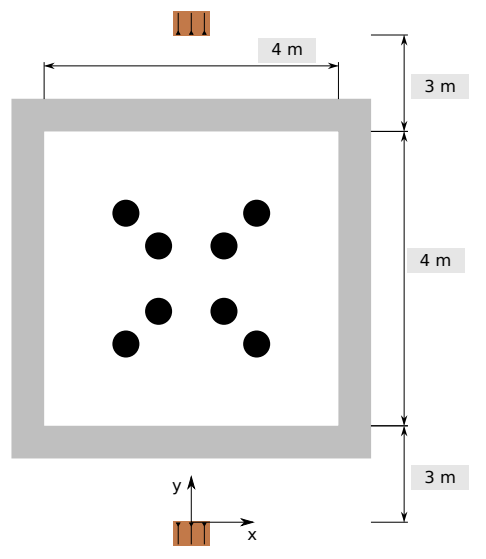

(a) Distributed units

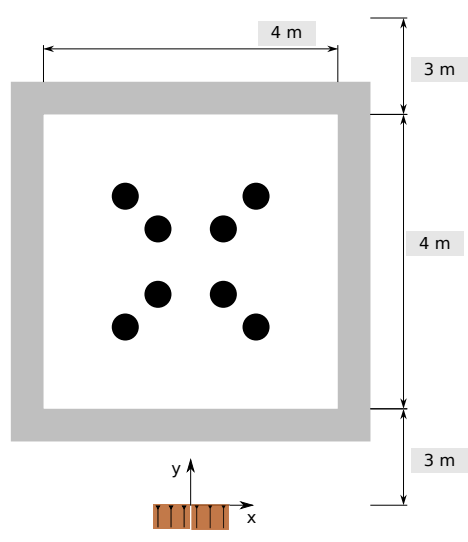

(b) Co-located units

Figure 4.16. Scene geometries for Scenario A.

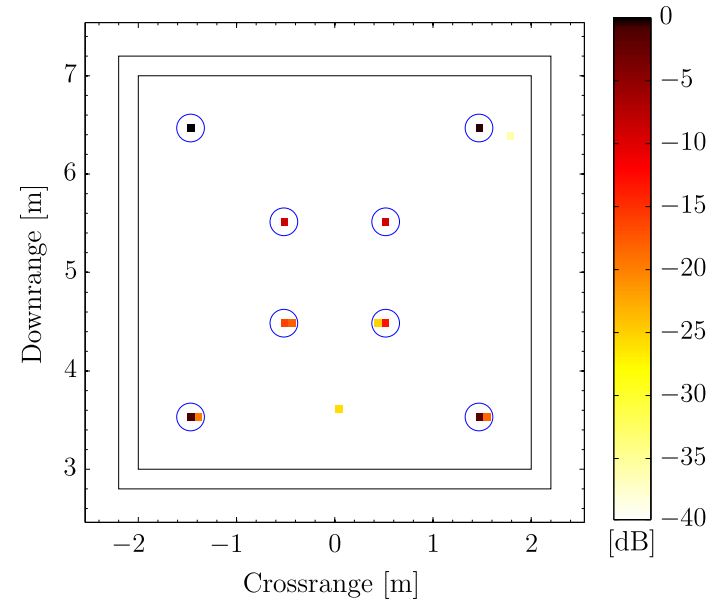

(a) Distributed

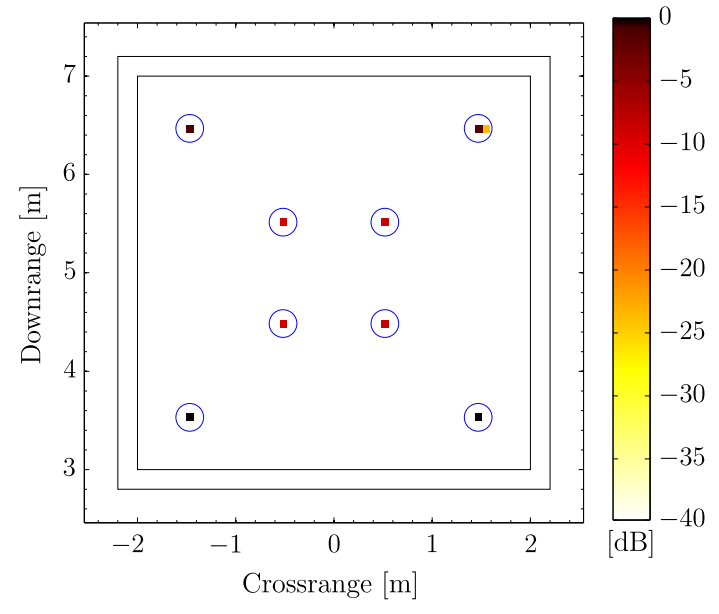

(b) Co-located

Figure 4.17. Reconstruction results for Scenario A: Opposing walls versus same wall placement.

Scenario B This scenario serves to compare co-located and distributed placements of two modules along a single wall. The number of targets for this case has been reduced to four. The targets are placed in pairs, with one target directly in front of the other for each pair. However, for now it is assumed that the wave can travel directly to the rear targets and is not shadowed by the targets in the front. The scene layout is illustrated in Figure 4.18. The corresponding images are shown in Figure 4.19. For the given scene, the reconstruction performance of the two radar configurations is equal and virtually perfect. 


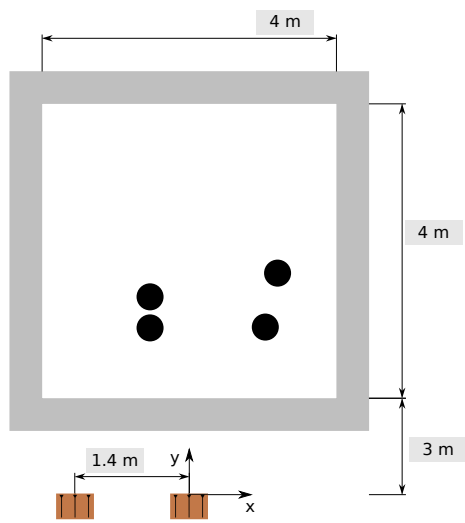

(a) Distributed units

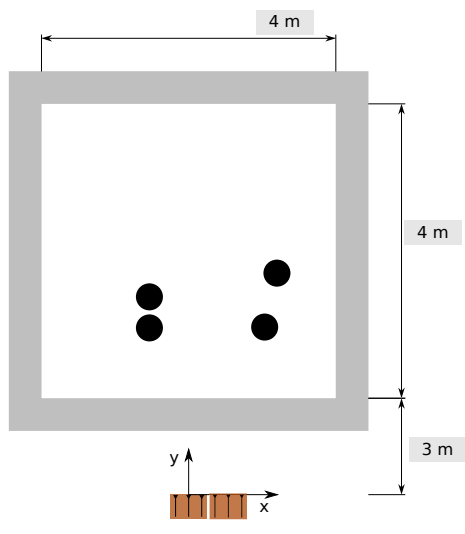

(b) Co-located units

Figure 4.18. Scene geometries for Scenarios B and C.

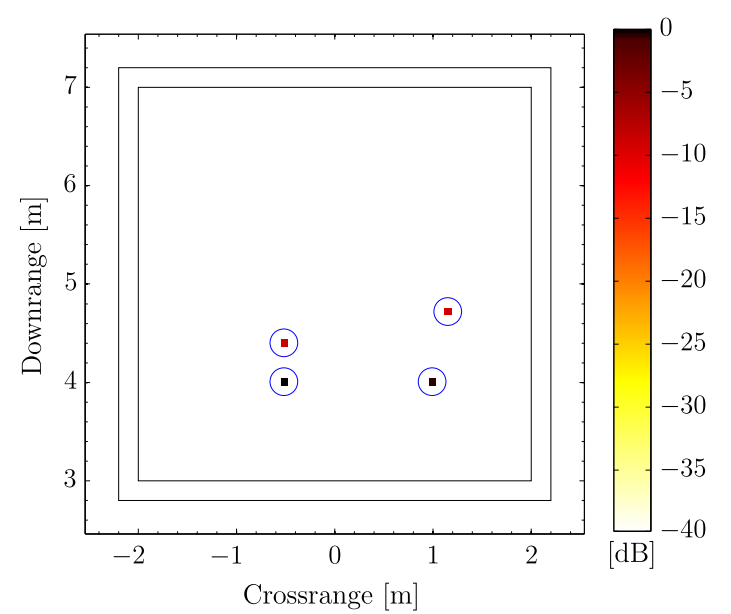

(a) Distributed

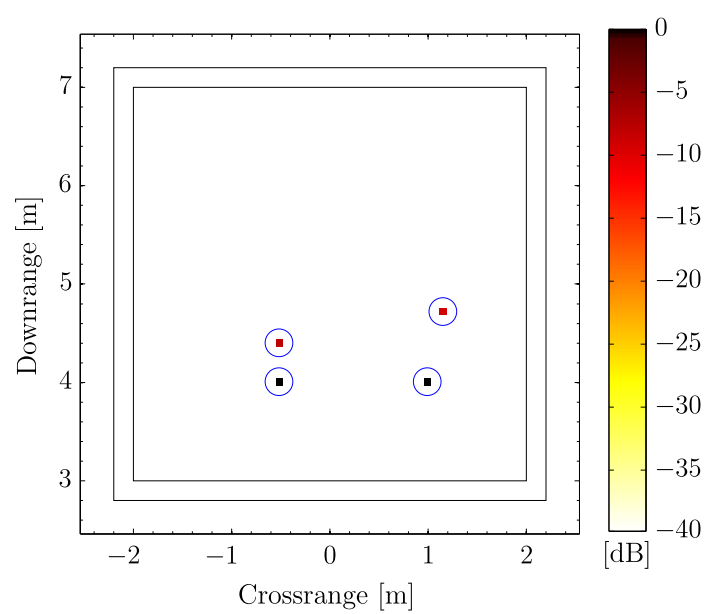

(b) Co-located

Figure 4.19. Reconstruction results for Scenario B: Widely-spaced versus closely-spaced placement without shadowing.

Scenario C The last scenario is designed to evaluate the impact of target shadowing on the imaging performance of distributed and co-located configurations. The layout of the scene is exactly the same as in Scenario B; however, shadowing of the two rear targets is included. In particular, the line-of-sight between the two rear targets and the radar module located at the origin is assumed to be blocked. This means that any path involving this line-of-sight is unavailable and weighted as zero, whereas indirect propagation via the side walls is observable. The second module does not suffer from shadowing. Hence, in total, 11 paths out of 28 ( 7 paths for 2 by 2 modules) are blocked in this scenario. Shadowing introduces two problems in the data measurements. First, the total energy returned from the shadowed targets is lowered leading to a decreased SNR. Second, the structural assumptions on the sparse solutions are violated. Since a 


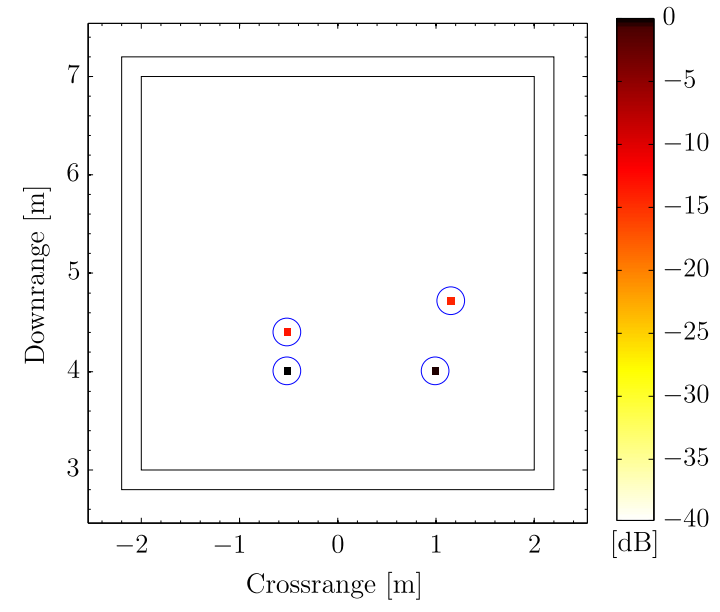

(a) Distributed

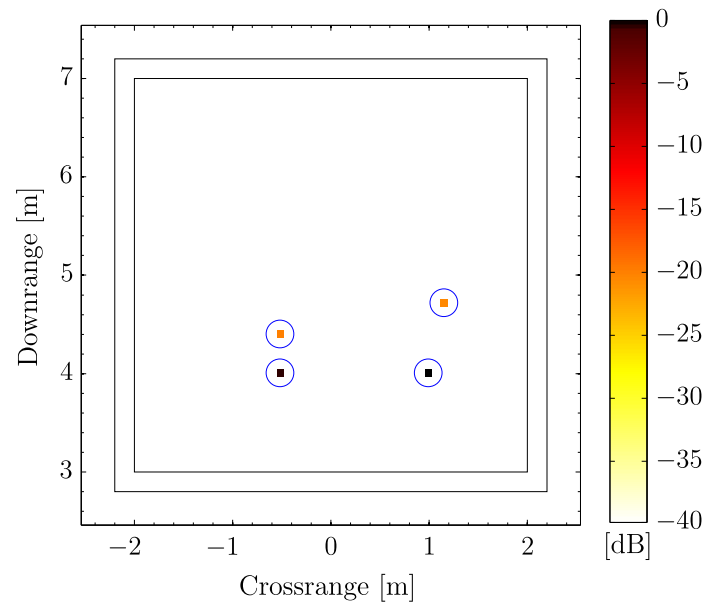

(b) Co-located

Figure 4.20. Reconstruction results for Scenario C: Widely-spaced versus closely-spaced placement including shadowing.

number of transmit-receive combinations are blocked, the common support assumption of the reflectivity vectors corresponding to various transmit-receive module pairs is no longer satisfied for the distributed configuration. In the co-located case, however, the assumption of invariant target reflectivity is violated as some transmit-receive pairs observe zero RCS. Figure 4.20 shows the example results for the two radar module placements of Scenario C. The four targets are correctly located for both radar configurations, however, the rear targets in the co-located case are significantly attenuated. It appears that violation of the softer group sparsity constraint is less damaging than the violation of the invariant target reflectivity. As such, despite the theoretical advantages of the co-located configuration under ideal conditions, the distributed configuration may be advantageous for recovering targets in shadowing situations.

Quantitative Results In order to obtain a quantitative comparison for the two radar module layouts, a full reference image quality metric is used. The ideal image, i.e., the ground truth, is compared with the reconstructions using the Earth Mover's Distance (EMD), which computes the amount of "dirt" or image intensity that has to be moved to get from one image to the other [RTG00]. It is a distance metric taking both the intensities and locations of significant image pixels into account. Thus, it is a suitable metric to assess image quality in a radar imaging context. All images are normalized to a maximum intensity of one; further, the pixel distance is also normalized to one and thresholded at five. That is, if the strongest pixel is off by one, the resulting EMD is also one. If the accumulated intensities of the two images differ, a penalty of five times this difference is added. The fast EMD implementation by Pele and Werman 
Table 4.2. EMD comparison across 50 Monte Carlo runs.

\begin{tabular}{|c|c|c|c|}
\hline & $\begin{array}{l}\text { Scenario A } \\
\text { dist co-loc }\end{array}$ & $\begin{array}{l}\text { Scenario B } \\
\text { dist co-loc }\end{array}$ & $\begin{array}{l}\text { Scenario C } \\
\text { dist co-loc }\end{array}$ \\
\hline & $19.01 \quad 3.89$ & $\begin{array}{ll}1.64 & 1.39\end{array}$ & $\begin{array}{ll}4.16 & 3.97\end{array}$ \\
\hline & 6.31 & $\begin{array}{ll}1.59 & 1.41\end{array}$ & $3.10 \quad 3.96$ \\
\hline std. dev. & 69.75 & $0.42 \quad 0.24$ & $7.15 \quad 0.26$ \\
\hline
\end{tabular}

[PW09] is used for the calculations.

The mean, median and standard deviation of the EMD for 50 Monte Carlo runs are listed in Table 4.2 for Scenarios A, B and C. Comparing the median EMD, one can observe that a co-located configuration yields better reconstruction results for Scenarios $\mathrm{A}$ and $\mathrm{B}$, where the assumptions on the structure of the solution hold. In Scenario $\mathrm{C}$, however, the distributed placement performs slightly better. The observation does not hold for the mean and the standard deviation in Scenario C, which indicates that the reconstruction may fail in some instances for the distributed radar. Hence, the additional spatial diversity comes at the cost of being more susceptible to measurement noise.

\subsection{Conclusions}

In this chapter, sparsity-based multipath exploitation with perfectly known room geometry was considered. Various methods have been proposed that reconstruct scenes with purely stationary targets, moving targets and multiple radar modules. It has been shown that properly considering multipath is essential for obtaining clean images without ghosting artifacts. Also, multipath contains additional information on the targets that can be used to one's advantage. In the moving target scenario, it has been demonstrated that multipath exploitation improves target velocity estimates over the direct path only case. The proposed framework is versatile, such that it can also be adapted to a multi-module radar imaging system. Theoretical analysis and simulations showed that a co-located is superior over a distributed placement. However, in difficult scenarios with target shadowing, a distributed radar system could provide more reliable imaging performance. All findings have been confirmed using simulated and measured data. 


\section{Chapter 5}

\section{Mitigating Wall Effects and Uncertainties}

This chapter deals with additional effects related to the building walls that have not been discussed yet. A short motivation regarding these issues, namely wall returns and wall location uncertainties is included in Section 5.1. So far, it was assumed that direct wall returns have been removed prior to image reconstruction. In a practical TWRI scenario, wall clutter mitigation is crucial to be able to process the comparably weak target returns. To this end, a CS-based approach is proposed in Section 5.2 that simultaneously separates and reconstructs the wall and target signals. Furthermore, the exact positions of the walls are usually not known beforehand. This knowledge is, however, critical for successful multipath mitigation. Hence, a joint wall position estimation and image reconstruction method is proposed in Section 5.3. The chapter is concluded in Section 5.4.

The material presented in this chapter is partly taken from [LAAZ13b, LAAZ14a, LAAZ14b, LAAZ14d, LZ14, LAAZ15d, LAAZ15c].

\subsection{Motivation}

In the previous chapter, direct returns from the front wall have not been considered. However, as explained in Section 3.4, the wall as an extended and strong reflector may mask and/or occupy a significant part of the scene of interest. From a CS point of view, the image becomes more populated, which adversely affects the reconstruction performance. When not mitigated, the front wall returns make conventional CS approaches fail [LAAN13a]. For illustration, a simulation of the beamformed image generated from the total signal model (3.21) including target returns, wall returns and multipath is shown in Figure 5.1. The scene consists of a single target at the circled location and the interior walls are indicated by the black line. The target contribution includes $R=6$ paths, whereas $R_{\mathrm{w}}=4$ propagation paths are considered for the front wall. The first wall echo is assumed to be twice the amplitude of the target return and subsequent wall reverberations are attenuated by factors of 0.4 . The first two returns lie at the inner and outer boundaries of the front wall. The higher order returns appear deeper inside the room and may interfere with targets located close to the wall. 


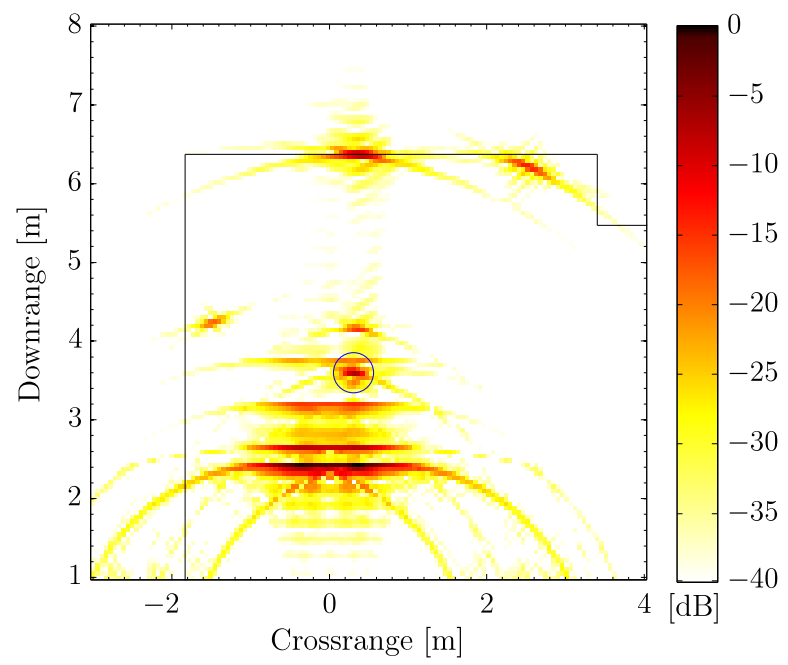

Figure 5.1. Beamformed image with wall and ghost targets taking $R=5$ and $R_{\mathrm{w}}=4$ propagation paths into account.

Existing wall-removal or wall-clutter mitigation schemes include background subtraction [AA08a, $\mathrm{MKA}^{+} 08$ ]. In this method, data of an empty scene without target is acquired and then coherently subtracted from the target scene measurements. Provided that background data is available, this method works well in sparse reconstruction [YA08, LDZ11]. Alternatively, spatial filtering [YA09] or subspace projection [TBA11] approaches have been developed for full data measurements. These methods have also been shown to perform well within the CS framework [LAAN13a]. In Section 5.2, a conceptually different approach is followed that jointly reconstructs the wall returns and the image [LAAZ14b].

Until now, it was assumed that the locations of the interior walls are known. In practice, precise prior knowledge of the interior wall locations is usually not available. The wall locations are usually estimated from the returns using building layout estimation techniques, such as [LAAN13b, vRdW14]. These estimates are subject to errors that can be on the order of TWRI system wavelengths. Multipath exploitation requires accurate knowledge of the room layout in order to deliver high quality images. For coherent processing and group sparse reconstruction, sub-wavelength accuracy of the propagation paths is necessary. Figure 5.2 illustrates the issue of inaccurate wall locations in multipath exploitation. The scenario includes four targets at the circled locations in a room with three walls and $R=7$ propagation paths involving reflections at the side walls. The true target locations are circled and the interior walls are depicted by black lines. In the reconstruction, the assumed wall locations are off by $0.1 \mathrm{~m}$ and $0.2 \mathrm{~m}$ for the right and left walls, respectively. This comparably small error results in a complete breakdown of the reconstruction method. 


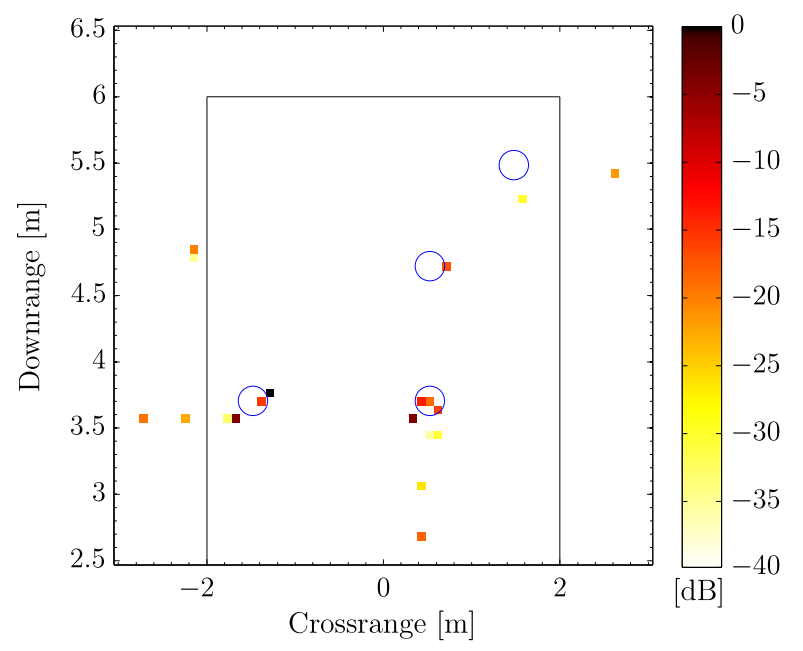

Figure 5.2. Multipath exploitation with erroneous wall locations.

Hence, wall locations should be estimated or corrected based on the acquired data. In Section 5.3, a method is proposed that simultaneously estimates wall locations and performs sparse reconstruction of the scene.

\subsection{Front Wall Reflections}

In this section, a CS-based approach is proposed that tackles the issue of strong front wall reflections. Instead of dealing with wall-clutter mitigation and imaging separately, both are achieved at the same time. The signal contributions of target and wall are separated utilizing a joint sparse reconstruction scheme. Wall and target returns are captured by two different signal models that are matched to the respective signal components. This section assumes a purely stationary scene and stepped-frequency measurements. When imaging moving targets, the stationary wall returns can usually be dealt with efficiently using Doppler processing [QAA13]. The major findings of this section were published in [LAAZ13b, LAAZ14b].

\subsubsection{Wall Reflection Model}

As discussed in Section 3.4, the wall returns consist of the direct returns and the reverberation. The wall part of the received signal is modeled as additive components from the wall surface and reverberations within the wall, refer to (3.18). 
For ease of notation and implementation, a modified wall model is used for the CS reconstruction. The wall is modeled as a composition of small wall segments that correspond to the grid of the target image. This gives the additional flexibility of modeling returns that do not originate from a perfectly homogeneous wall. Further, despite assuming knowledge of the front wall parameters, these parameters are usually estimates that contain errors. The proposed model has sufficient degrees of freedom to represent the wall returns when dealing with errors in the wall parameters. This is an advantage over modeling and coherent subtraction of the wall returns [DS08], as the coherent subtraction may fail even for small deviations of the parameters.

The vectorized wall reverberation model, similar to (3.17), can be expressed as

$$
\boldsymbol{z}_{\mathrm{FD}, \mathrm{w}}=\boldsymbol{\Psi}_{\mathrm{w}}^{(0)} \boldsymbol{\sigma}_{\mathrm{w}}^{(0)}+\boldsymbol{\Psi}_{\mathrm{w}}^{(1)} \boldsymbol{\sigma}_{\mathrm{w}}^{(1)}+\cdots+\boldsymbol{\Psi}_{\mathrm{w}}^{\left(R_{\mathrm{w}}-1\right)} \boldsymbol{\sigma}_{\mathrm{w}}^{\left(R_{\mathrm{w}}-1\right)}
$$

where $\boldsymbol{\sigma}_{\mathrm{w}}^{(r)}$ are the vectorized reflectivities of the wall segments and $\boldsymbol{\Psi}_{\mathrm{w}}^{(r)}$ contain the delay information for the $r$-th path. The matrices $\Psi_{\mathrm{w}}^{(r)}$ are similar to $\Psi_{\mathrm{FD}}^{(r)}$, however, only the contributions of specular reflections are retained. This can be described by a masking operation,

$$
\Psi_{\mathrm{w}}^{(r)}=\boldsymbol{M}^{(r)} \circ \Psi_{\mathrm{FD}}^{(r)},
$$

where $\boldsymbol{M}^{(r)} \in\{0,1\}^{L M N \times P_{x} P_{y}}$ are binary matrices. An element $\left[\boldsymbol{M}^{(r)}\right]_{i p}$ is equal to one if the $p$-th wall segment is visible by a transmitter/receiver pair corresponding to the $i$-th row via the $r$-th path and zero otherwise. Hence, for every element in $\boldsymbol{M}^{(r)}$ the specular reflection condition, as valid for wall returns, needs to be checked. If the angle of incidence of the transmit path and the angle of reflection of the receive path are equal, the corresponding entry is one and zero otherwise. In the monostatic case, the condition can be simplified, i.e., the contribution from all specular targets that are not directly in front of the transceiver should be masked out by $\boldsymbol{M}^{(r)}$. Any wall segment that is not exactly in the broadside direction of the array is not visible, as the transmitted wave will be reflected away from the transceiver.

The downsampled wall returns are denoted as

$$
\overline{\boldsymbol{z}}_{\mathrm{FD}, \mathrm{w}}=\boldsymbol{\Phi}_{\mathrm{FD}} \boldsymbol{z}_{\mathrm{FD}, \mathrm{w}}
$$

using an undersampling scheme as described in Section 3.5. Hence, the superposition of target and wall contributions in the measured signal as received by the array yields

$$
\overline{\boldsymbol{z}}_{\mathrm{FD}}=\overline{\boldsymbol{z}}_{\mathrm{FD}, \mathrm{t}}+\overline{\boldsymbol{z}}_{\mathrm{FD}, \mathrm{w}}+\boldsymbol{n}_{\mathrm{FD}},
$$

where the target contribution $\overline{\boldsymbol{z}}_{\mathrm{FD}, \mathrm{t}}$ is defined in (3.23). 


\subsubsection{Separate Reconstruction}

Making use of the two models describing target and wall returns, a sparse reconstruction of the scene is sought. First, a naïve approach is discussed which reconstructs the wall and target images separately.

The group sparsity-based multipath exploitation approach (4.11), as described in Section 4.3, can be utilized. This method is applied twice, first to reconstruct the target scene using model (3.23) and then to reconstruct the wall using model (5.3). Hence, from the measurements $\overline{\boldsymbol{z}}_{\mathrm{FD}}$ two images, $\hat{\boldsymbol{\sigma}}$ and $\hat{\boldsymbol{\sigma}}_{\mathrm{w}}$, can be reconstructed to describe the targets and the wall, respectively. Note that the two reconstructions are independent in the sense that no information from the wall image is used to form the target image and vice-versa.

\subsubsection{Joint Group Sparse Reconstruction}

A more sophisticated joint reconstruction approach was proposed in [LAAZ14b] utilizing the knowledge of superimposed target and wall returns. To this end, the idea of stacking the models from GSCS as described in Section 4.3.2 is adopted. The wall and target models (3.23) and (5.3) are combined, resulting in

$$
\overline{\boldsymbol{z}}_{\mathrm{FD}}=\boldsymbol{\Phi}_{\mathrm{FD}} \tilde{\boldsymbol{\Psi}}_{\mathrm{j}} \tilde{\boldsymbol{\sigma}}_{\mathrm{j}}+\boldsymbol{n}_{\mathrm{FD}} .
$$

The vector $\tilde{\boldsymbol{\sigma}}_{\mathrm{j}} \in \mathbb{C}^{P_{x} P_{y}\left(R+R_{\mathrm{w}}\right) \times 1}$ is obtained by stacking the various vectors of the scene of interest, i.e.,

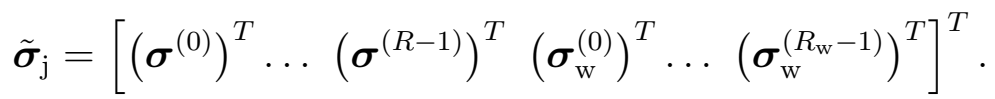

The overall measurement matrix has the form

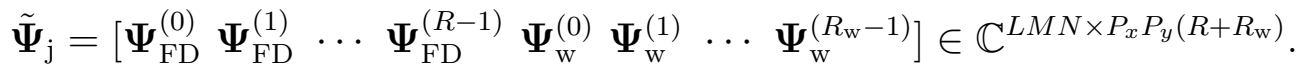

Using the above high-dimensional joint model in (5.5) to (5.7), the mixed-norm regularized reconstruction problem is posed as

$$
\hat{\tilde{\boldsymbol{\sigma}}}_{\mathrm{j}}=\arg \min _{\tilde{\boldsymbol{\sigma}}_{\mathrm{j}}} \frac{1}{2}\left\|\overline{\boldsymbol{z}}_{\mathrm{FD}}-\boldsymbol{\Phi}_{\mathrm{FD}} \tilde{\boldsymbol{\Psi}}_{\mathrm{j}} \tilde{\boldsymbol{\sigma}}_{\mathrm{j}}\right\|_{2}^{2}+\lambda \zeta_{\mathrm{j}}\left(\tilde{\boldsymbol{\sigma}}_{\mathrm{j}}\right),
$$

where

$$
\zeta_{\mathbf{j}}\left(\tilde{\boldsymbol{\sigma}}_{\mathrm{j}}\right):=\sum_{p=0}^{P_{x} P_{y}-1}\left\|\left[\sigma_{p}^{(0)}, \sigma_{p}^{(1)}, \ldots, \sigma_{p}^{(R-1)}\right]^{T}\right\|_{2}+\sum_{p=0}^{P_{x} P_{y}-1}\left\|\left[\sigma_{\mathrm{w}, p}^{(0)}, \sigma_{\mathrm{w}, p}^{(1)}, \ldots, \sigma_{\mathrm{w}, p}^{\left(R_{\mathrm{w}}-1\right)}\right]^{T}\right\|_{2} .
$$


Note that the regularizer in (5.9) is different from the target only case (4.12). The regularization enforces group sparsity for targets, as reflected in the first part which is equivalent to (4.12). The sub-images of the wall follow the same sparsity structure, since all reverberations originate from the same physical wall. This gives rise to the property that all wall images share the same support. However, no interrelations between wall and target sub-images are desired. Consequently, both are regularized by separate terms in order to achieve the goal of separating their respective return signals.

Instead of using a single regularization parameter $\lambda$ to adjust the sparsity of the solution, separate parameters could be used for the two parts in (5.9). The potential performance benefits are, however, offset by the problem of selecting proper values of the regularization parameters which is critical for good performance.

Note that in (5.5), the measurement or dictionary matrix is extended to include both target and wall atoms for all possible paths. Using this dictionary the wall and target contributions in $\overline{\boldsymbol{z}}_{\mathrm{FD}}$ can be expressed in a sparse fashion. However, as the wall and target measurement matrices are very similar, considerable mutual coherence between their columns is observed. This might adversely affect the reconstruction as the wall and target contributions may not get fully separated. A more detailed study of the mutual coherence of the two models can be found in [LAAZ14b].

In order to obtain a final image, the results from (5.8) have to be accumulated. A non-coherent combination is performed separately for the target and wall parts of the solution obtaining a final target image $\hat{\tilde{\boldsymbol{\sigma}}}_{\mathrm{GS}}$ and a final wall image $\hat{\tilde{\boldsymbol{\sigma}}}_{\mathrm{GS}, \mathrm{w}}$.

\subsubsection{Joint Overlapping Group Sparse Reconstruction}

Finally, the overlapping group sparse approach as described in Section 4.3 .3 can be extended to reconstruct wall and target images. This is a generalization of the nonoverlapping groups in the previous subsection and allows to include appropriate prior knowledge of neighborhood information on the target and wall images separately. For the target sub-images, an isotropic first-order neighborhood is used as introduced in Section 4.3.3. However, for the wall sub-images, the wall pixels form lines along the crossrange direction. Hence, a group structure that promotes pixel groups elongated in crossrange direction is chosen.

Considering the above observations, the regularizer (5.9) is replaced by

$$
\zeta_{\mathbf{j}}\left(\tilde{\boldsymbol{\sigma}}_{\mathrm{j}}\right):=\sum_{p=0}^{P_{x} P_{y}-1} \sqrt{\sum_{r=0}^{R-1}\left\|\boldsymbol{W}_{\mathrm{t}}^{(p)} \sigma_{g_{p}}^{(r)}\right\|_{2}^{2}}+\sum_{p=0}^{P_{x} P_{y}-1} \sqrt{\sum_{r=0}^{R_{\mathrm{w}}-1}\left\|\boldsymbol{W}_{\mathrm{w}}^{(p)} \sigma_{\mathrm{w}, h_{p}}^{(r)}\right\|_{2}^{2}},
$$



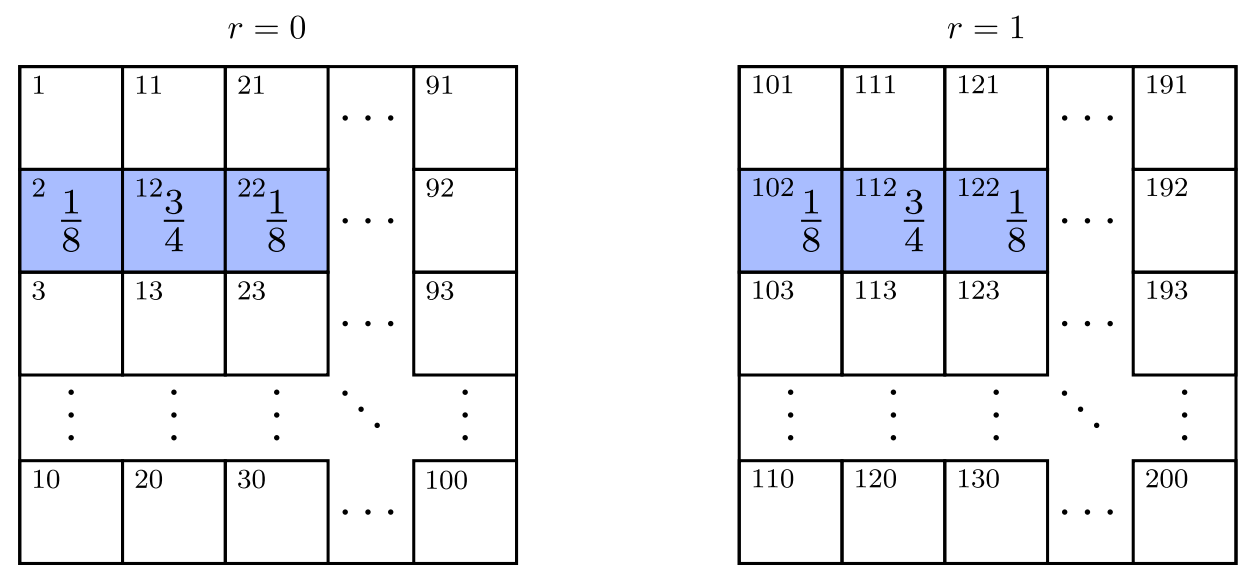

Figure 5.3. Illustration of a neighborhood grouping matched to walls. The left image corresponds to the direct path and the right image corresponds to multipath.

where the first term in (5.10) ensures the proper regularization for the target images and the second term for the wall images. Similar to the target only case, $g_{p} \subseteq\left\{0,1, \ldots, R P_{x} P_{y}-1\right\}, h_{p} \subseteq\left\{0,1, \ldots, R_{\mathrm{w}} P_{x} P_{y}-1\right\}$ are index sets corresponding to the group of pixels forming a neighborhood around pixel $p$. The diagonal weighting matrices $\boldsymbol{W}_{\mathrm{t}}^{(p)} \in \mathbb{R}^{\left|g_{p}\right| \times\left|g_{p}\right|}, \boldsymbol{W}_{\mathrm{w}}^{(p)} \in \mathbb{R}^{\left|h_{p}\right| \times\left|h_{p}\right|}$ ensure the weighting within a group according to the desired pixel neighborhood relation.

Figure 5.3 illustrates an example of the pixel grouping for walls. Again, the small number in the top left corner of each square indicates the pixel index and the large number in the center is the pixel weight of the depicted group. Assume we have an $P_{x} \times P_{y}=10 \times 10$ pixel image and $R_{\mathrm{w}}=2$ possible propagation paths. The index set for the 12th group, as shown in the figure, would be $h_{12}=\{2,12,22,102,112,122\}$, where the second part of the set corresponds to indices in the wall reverberation image $(r=1)$. The corresponding weighing matrix would be $\boldsymbol{W}_{\mathrm{w}}^{(12)}=\frac{1}{2} \operatorname{diag}\left(\frac{1}{8}, \frac{3}{4}, \frac{1}{8}, \frac{1}{8}, \frac{3}{4}, \frac{1}{8}\right)$. The index sets for all other groups are obtained similarly by shifting the rectangular mask to the appropriate pixel position. For target pixel grouping, the same index sets $g_{p}$ and weighing matrices $\boldsymbol{W}_{\mathrm{t}}^{(p)}$ are used as in Section 4.3.3.

\subsubsection{Simulation and Experimental Results}

Example results for reconstructing the target image along with the wall are presented. The same radar and scene geometry as in Section 4.3.4 is used. The key parameters are monostatic operation with $N=77$ transceivers, a bandwidth of $2 \mathrm{GHz}$ and $R=5$ multipath components. Again, the region of interest is discretized into $P_{x} \times P_{y}=$ 


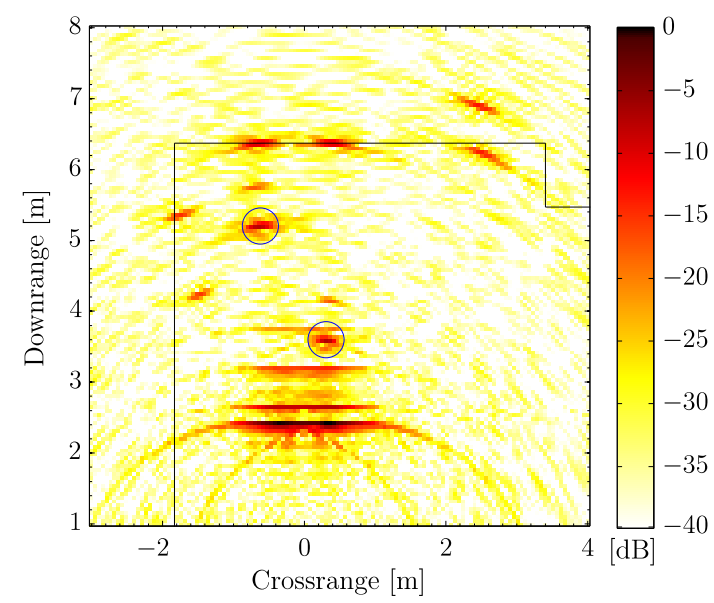

Figure 5.4. Conventional DSBF reconstruction of the simulated scene including the wall response.

$64 \times 64$ pixels. The returns from the front wall, including reverberations are taken into consideration this time.

\section{Simulation Results}

The received signals are simulated with a total of $R_{\mathrm{w}}=4$ propagation paths for the wall model. The direct wall response is assumed to be $6 \mathrm{~dB}$ stronger than the target returns. Subsequent wall returns are attenuated by $8 \mathrm{~dB}$ for each reverberation within the wall. White Gaussian noise with $0 \mathrm{~dB}$ SNR is added to simulated measurements.

The beamforming result using full measurements is depicted in Figure 5.4. One can clearly see that the first two wall returns lie at the inner and outer surface of the front wall. However, the higher order returns appear inside the room and potentially mask the targets at those locations.

One-fourth of the array elements and one-fourth of the frequencies are used for CS reconstruction. The corresponding results, averaged over 100 Monte Carlo runs, are displayed in Figure 5.5. Separate reconstruction of the wall and target images with multipath exploitation is shown in Figure 5.5a,b. The targets are reconstructed and the ghost targets are well suppressed. However, the wall response appears very strong in the target image as it is treated as a target response. Employing joint group sparse CS reconstruction as described in Section 5.2.3, a very clean reconstruction of the two targets is achieved, see Figure 5.5c. With this reconstruction approach, the ghost targets and the wall returns are well suppressed. Using overlapping groups results in 


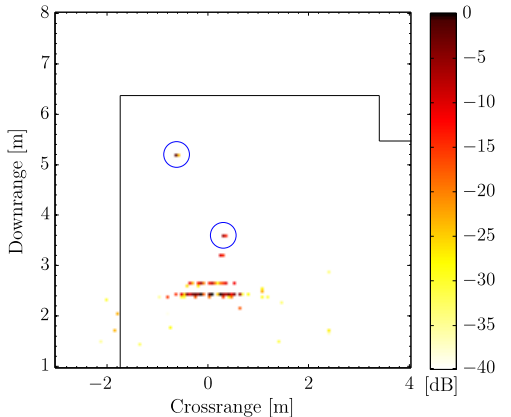

(a) Separate Reconstruction, Target

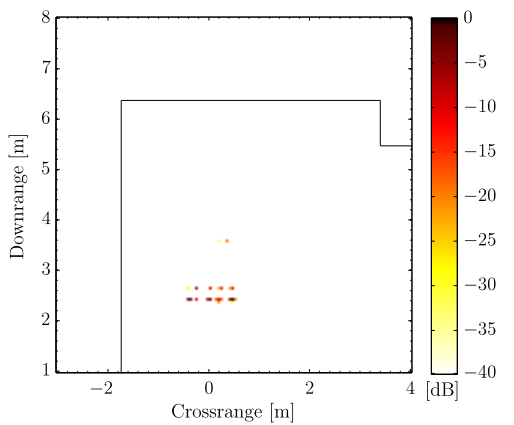

(b) Separate Reconstruction, Wall

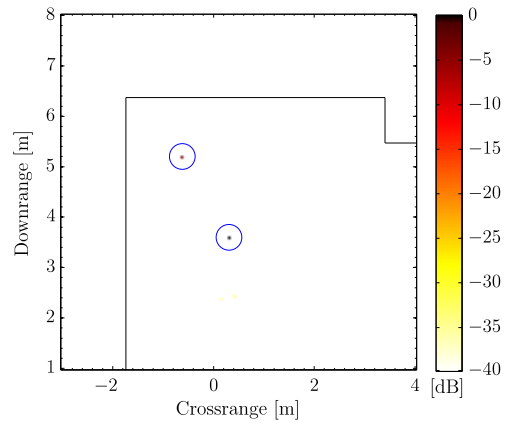

(c) Joint Non-overlapping GS Reconstruction, Target

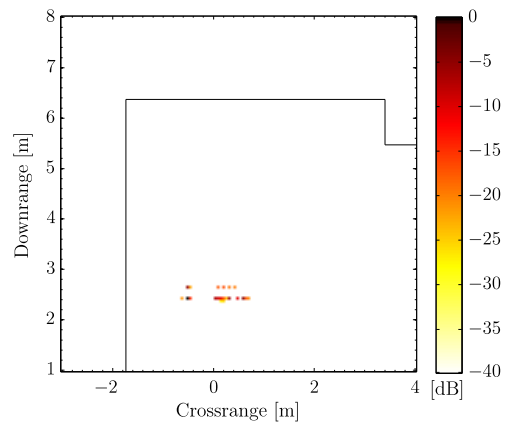

(d) Joint Non-overlapping GS Reconstruction, Wall

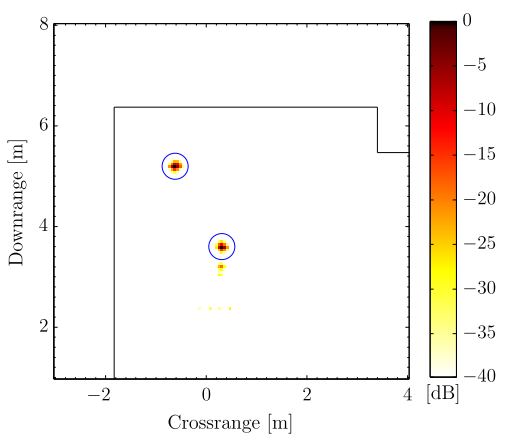

(e) Joint Overlapping GS Reconstruction, Target

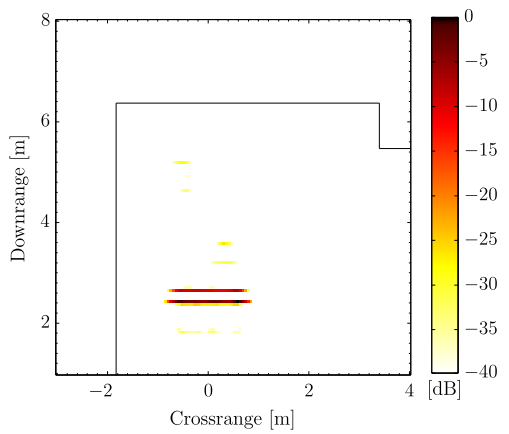

(f) Joint Overlapping GS Reconstruction, Wall

Figure 5.5. Reconstruction results using different algorithms for the simulated scene including the wall response. One fourth of both the array elements and frequency bins were used for the image formation.

a clean image featuring a certain smoothing of the targets, see Figure 5.5e. However, a weak artifact appears close to the target in the front. The reconstruction of the wall is very similar for separate reconstruction and joint non-overlapping group sparse CS reconstruction, refer to Figures 5.5b,d. The wall response appears in isolated pixels, roughly aligned in two lines. The depiction of the wall can be significantly improved by using the overlapping group sparse approach, see Figure 5.5f. The wall pixels form nicely connected lines in the image. Note that the first reverberation, corresponding to the return from the back face of the wall, is treated as a valid target and not as a multipath return. Hence, it appears in the reconstructed wall images as a second line.

\section{Experimental Results}

Experimental data from the Radar Imaging Lab of the scene with a single aluminum pipe as presented in Section 4.3.4 is used. No background subtraction is performed, hence, all wall returns are contained in the measurements. A Hamming window across 


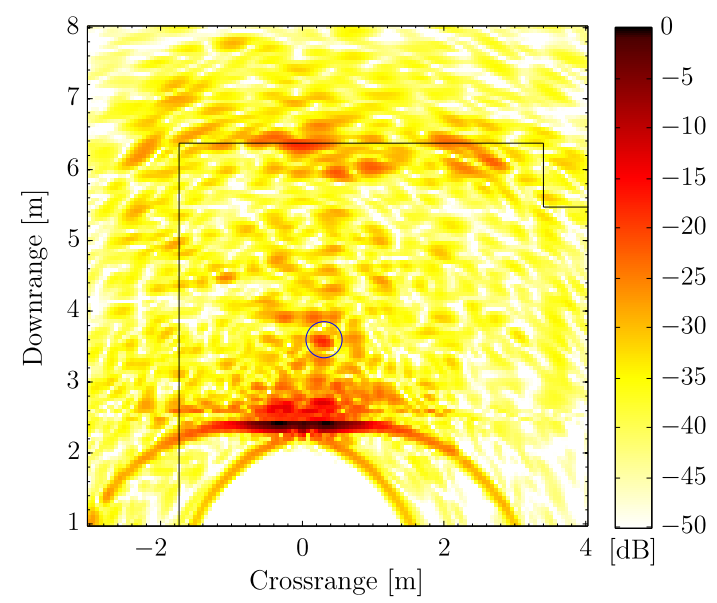

Figure 5.6. Conventional DSBF reconstruction of the $\mathrm{Al}$ pipe scene without using background subtraction.

the frequencies is applied to mitigate the effect of antenna mismatch. Additionally, time samples whose delays do not correspond to returns from the front wall or the room are gated out to clean the data.

Figure 5.6 depicts the beamformed image using all available data. The wall responses from the front face and the back face of the wall are clearly visible. Furthermore, one can recognize multipath propagation via the back wall of the room. Thus, only the direct path and one multipath via the back wall are modeled in the CS reconstruction algorithms.

In the sequel, downsampling to one fourth of the array elements and half of the frequency bins is considered. In Figures 5.7a,b, the separate CS reconstructions of the target and wall scenes are shown. The results for the joint group sparse CS method with non-overlapping and overlapping groups are depicted in Figures 5.7c,d and Figures $5.7 \mathrm{e}$,f, respectively. When applying the separate reconstruction approach, the target is very difficult to recognize due to the strong wall response in the target image. The target is increasingly better discerned for joint reconstruction with non-overlapping and overlapping groups. The target images show less clutter pixels beyond the front wall. However, none of the methods is able to separate the wall and target images in a satisfactory way. The reconstruction of the wall image is significantly better for the overlapping group sparse reconstruction as compared to the other two approaches. The limited capability to separate the wall and the target responses can be attributed to two issues. First, there is residual sidelobe leakage, owing to the very strong antenna mismatch. This effect is not modeled in the received signal and, hence, impairs the CS reconstruction. Second, the front wall is constructed by stacking solid concrete blocks 


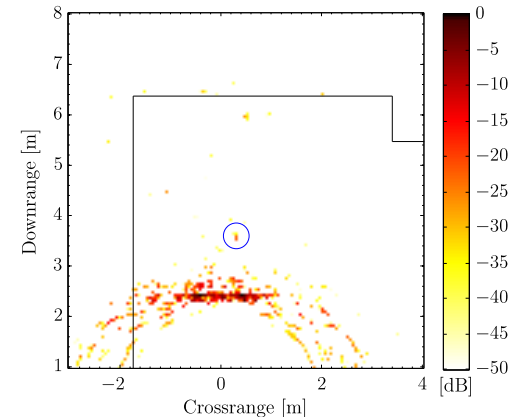

(a) Separate Reconstruction, Target

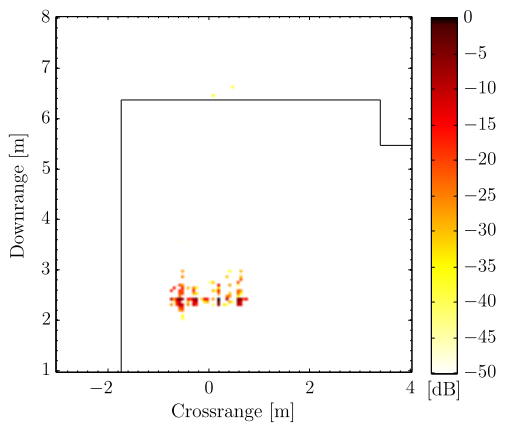

(b) Separate Reconstruction, Wall

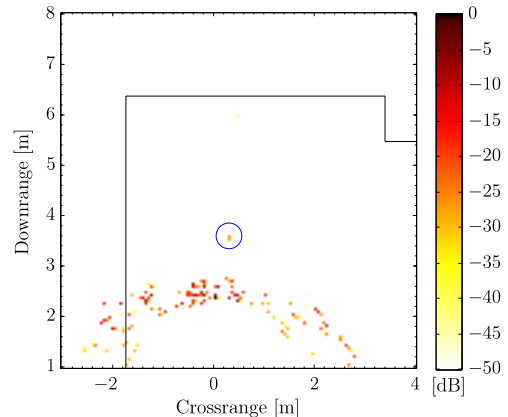

(c) Joint Non-overlapping GS Reconstruction, Target

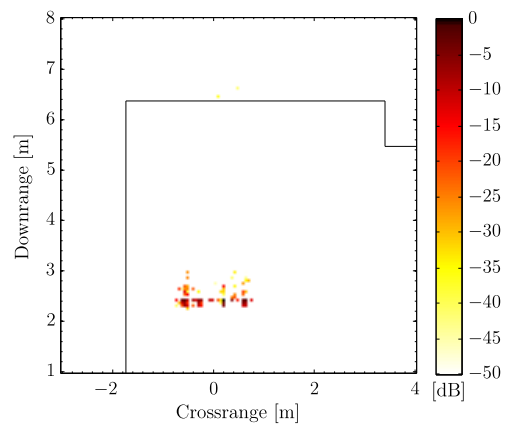

(d) Joint Non-overlapping GS Reconstruction, Wall

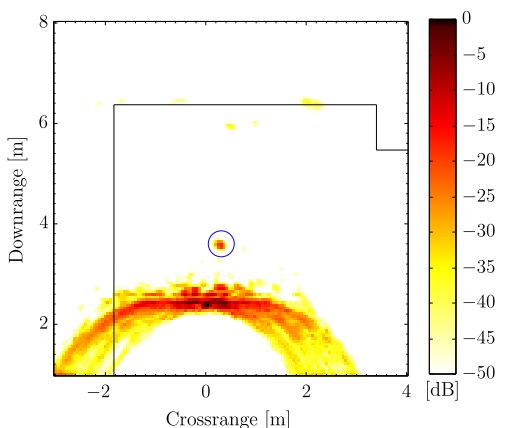

(e) Joint Overlapping GS Reconstruction, Target

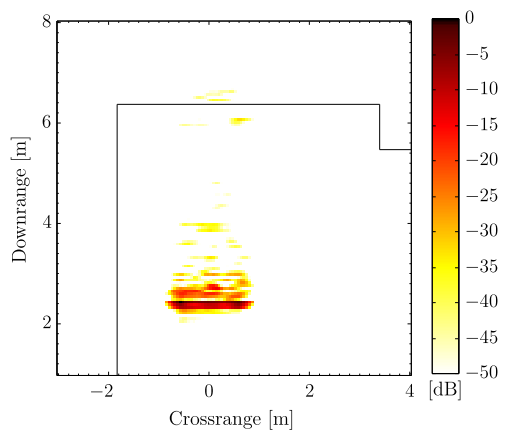

(f) Joint Overlapping GS Reconstruction, Wall

Figure 5.7. Reconstruction results using different algorithms for the aluminum pipe scene without background subtraction. One fourth of the array elements and half of the frequency bins were used for the image formation.

without any mortar or plaster, resulting in a wall that has air gaps and no smooth surface. Therefore, the assumptions of homogeneity and specular reflection for the front wall are violated. This leads to a mismatch between the wall model and the actual wall returns, which possibly results in a strong leakage of the wall into the target image.

\subsection{Wall Location Correction}

This section deals with multipath exploitation under wall location errors. A received signal model is developed taking wall location uncertainties into account. Based on this model, a joint image reconstruction and wall location correction method is proposed. This method ensures high image quality for multipath exploitation with inaccurate knowledge of wall locations. The results discussed here were reported in [LAAZ14a, LAAZ15d]. 


\subsubsection{Multipath Model Including Wall Position Errors}

In order to deal with wall location errors, the positions of the interior walls are parametrized. Considering a rectangular room, the location of each wall can be described by a scalar parameter, i.e., the oriented distance to the center of the room $w_{i}, i=1, \ldots, 4$. The wall location parameters are stacked in a single wall position vector $\boldsymbol{w}$. The dictionaries, in turn, now depend on the wall locations, resulting in parametrized dictionaries $\Psi^{(r)}(\boldsymbol{w}), r=1, \ldots, R-1$. Note that the dictionary $\boldsymbol{\Psi}^{(0)}$ associated with the direct path does not depend on $\boldsymbol{w}$. Further, the multipath dictionaries depend only the entries of $\boldsymbol{w}$ corresponding to walls involved in specular reflection. The dictionaries are defined by (3.5), however, multipath propagation and the wall position has to be taken into account when calculating the propagation delays. Note that the propagation delays and, in turn, the dictionaries depend non-linearly on the wall locations $\boldsymbol{w}$. Finally, the received signal model under wall location errors can be written as

$$
\boldsymbol{z}=\boldsymbol{\Psi}^{(0)} \boldsymbol{\sigma}^{(0)}+\boldsymbol{\Psi}^{(1)}(\boldsymbol{w}) \boldsymbol{\sigma}^{(1)}+\cdots+\Psi^{(R-1)}(\boldsymbol{w}) \boldsymbol{\sigma}^{(R-1)} .
$$

Note that the structure of (5.11) is equivalent to the measurement model (3.16) in the case that the wall positions are known a priori. Similar to the previous sections, a compact notation for the model is introduced. The composite dictionary $\tilde{\boldsymbol{\Psi}}(\boldsymbol{w})=\left[\begin{array}{llll}\Psi^{(0)} & \Psi^{(1)}(\boldsymbol{w}) & \cdots & \Psi^{(R-1)}(\boldsymbol{w})\end{array}\right]$ is defined as the concatenation of the individual dictionaries corresponding to the $R$ paths. After applying an undersampling operation, the received signal including noise reads

$$
\overline{\boldsymbol{z}}=\boldsymbol{\Phi} \boldsymbol{z}+\boldsymbol{n}=\boldsymbol{\Phi} \tilde{\boldsymbol{\Psi}}(\boldsymbol{w}) \tilde{\boldsymbol{\sigma}}+\boldsymbol{n} .
$$

The typical structure of $\boldsymbol{\Phi}$ has been discussed in Section 3.5. If no downsampling in time or space is desired, $\boldsymbol{\Phi}$ can be set as the identity matrix.

In the presence of wall location errors, two mechanisms lead to degradation of the reconstructed image quality. First, the returns from a specific multipath are coherently combined in the measurement model. Since the wall location errors cause the expected multipath delays to deviate from the actual delays, the coherence of the multipath returns is lost, resulting in a mismatch between the dictionary $\tilde{\boldsymbol{\Psi}}(\boldsymbol{w})$ and the received signal. Second, wall location errors may lead to a misalignment of the various subimages in the signal model, thereby violating the group sparse structure of $\boldsymbol{\sigma}$. The perfect alignment of the sub-images is only guaranteed if the predicted multipath delays and, in turn, the wall locations are accurate.

Finally, a distributed radar configuration with multiple modules may be considered as described in Section 4.5. This leads to a model corresponding to (4.40), however, 
including wall errors. The received signal for the general multiple module case can be expressed as

$$
\breve{z}=\breve{\Phi} \breve{\Psi}(\boldsymbol{w}) \breve{\sigma}+\tilde{n} .
$$

Note that the only difference is the wall position parameter $\boldsymbol{w}$, the structure of the $(\breve{\cdot})$ variables is unchanged. As before, co-located and distributed placement of the radar modules is considered. The former allows coherent processing of all returns, whereas coherence is lost for the latter in exchange for higher spatial diversity.

\subsubsection{Joint Sparse Reconstruction and Wall Position Estima- tion}

In order to deal with the aforementioned issues dictionary and support mismatch, it is imperative to take wall position uncertainties into account in the reconstruction process. An approach for joint image reconstruction and wall location estimation is proposed, i.e., a joint minimization over the reflectivity vector $\breve{\boldsymbol{\sigma}}$ and the wall position vector $\boldsymbol{w}$

$$
\min _{\boldsymbol{w}, \breve{\boldsymbol{\sigma}}} \frac{1}{2}\|\breve{\boldsymbol{z}}-\breve{\boldsymbol{\Phi}} \breve{\Psi}(\boldsymbol{w}) \breve{\boldsymbol{\sigma}}\|_{2}^{2}+\lambda\|\breve{\boldsymbol{\sigma}}\|_{2,1} .
$$

Note that the reconstruction problem (5.14) is now a non-convex optimization problem as the dictionary depends non-linearly on the wall locations. In fact, in the near-field imaging case, there is not even a closed-form solution for the delays contained in the matrix [AA08a], which renders the problem difficult to solve directly. However, it is always possible to minimize over some variables first and then minimize over the remaining ones [BV04, Ch. 4, p. 133]. Choosing to optimize over $\breve{\boldsymbol{\sigma}}$ first and then over $\boldsymbol{w},(5.14)$ can equivalently be posed as [LAAZ14a]

$$
\min _{\boldsymbol{w}} \min _{\breve{\boldsymbol{\sigma}}} \frac{1}{2}\|\breve{\boldsymbol{z}}-\breve{\boldsymbol{\Phi}} \breve{\boldsymbol{\Psi}}(\boldsymbol{w}) \breve{\boldsymbol{\sigma}}\|_{2}^{2}+\lambda\|\breve{\boldsymbol{\sigma}}\|_{2,1}
$$

The overall optimization problem is still non-convex, but it now consists of a convex part and a non-convex part. More specifically, the inner optimization over $\breve{\boldsymbol{\sigma}}$ is convex, which is exactly the same as the reconstruction problem (4.41) and can be solved efficiently. The outer minimization is non-convex; however, the dimension of the solution space is much smaller and, thus, easier to search. In a typical room, the number of unknown interior wall locations is at the most three, whereas the number of grid points is several orders of magnitude larger. Note that (5.15) can be seen as a highlyparametrized dictionary learning problem. The parameters $\boldsymbol{w}$ should be learned, such that the sparsest representation of the observed scene is achieved. The high degree of parametrization comes from the physical aspects of wave propagation in the indoor environment, thereby restricting the number of degrees of freedom. Further, although the 
nested optimization problem is posed for the multiple module case, it is still applicable to reconstruction under wall location uncertainties as well using a single module. This is because the single unit module is a special case of the co-located multiple module configuration with $S=1$.

The outer nonconvex problem in (5.15) can be solved by general nonlinear optimization methods. As there is no closed-form method to compute the dictionary, it is not feasible to find an analytic solution for the gradient of the problem. As such, possible candidates are, among others, Quasi-Newton (QN) methods using finite-difference gradients [GMW81] or heuristic methods, such as Particle Swarm Optimization (PSO) [KE95, PKB07]. In order to improve the convergence for any method, the search space is limited to a feasible region. Assuming that a building layout estimation technique has been applied to the data beforehand yielding estimates of the wall locations, which are within a $0.5 \mathrm{~m}$ error margin. The specific error margin is chosen in accordance with the specifications of the VisiBuilding Program by the U.S. Defense Advanced Research Projects Agency [Bar06]. Hence, the search space is limited by box constraints centered around the initial estimate of the wall locations. Note that accurate knowledge of the front wall thickness and permittivity is assumed, however, the proposed approach could be extended to include these parameters. The benefit of the QN method is its guaranteed convergence. However, the solutions of the inner convex optimization problem must be very accurate in order to prevent erroneous finite-difference estimates of the gradient for QN methods. Provided that the estimates of the gradient are sufficiently accurate, the objective value decreases at every iteration. As the objective function in (5.15) is bounded below, the algorithm will always converge to a local minimum. Since the objective function is non-convex and, therefore, may have many local minima, finding a local minimum is not equivalent to finding the global minimum. In general, no claims about the optimality of the found local minimum can be made. In contrast to that, being a heuristic method, PSO has no performance guarantees. However, it does provide a certain probability of overcoming local minima and descending closer to the global minimum. This may lead to better reconstruction performance at the cost of higher computational complexity.

The computational complexity of the reconstruction mainly lies in the calculation of the propagation delays for the dictionaries and in evaluating the matrix-vector multiplications in equations (4.27) and (4.29) or their adjoint versions. In order to solve one instance of (4.41), the delays should be calculated once, whereas the matrix-vector multiplications are carried out multiple times due to the iterative nature of the available solvers. Hence, it is reasonable to compare the QN method with PSO by counting the number of mixed-norm problems (4.41) that need to be solved. Extensive simulations showed that PSO usually requires roughly ten times the number of solved sub-problems 
as compared to QN method. In general, the wall error correction method results in much higher numerical cost as compared to the known wall location case.

\subsubsection{Simulation and Experimental Results}

\section{Simulation Results}

The same simulation setup as in the distributed radar case with known wall positions is used as presented in Section 4.5.4. The nominal size of the room is $4 \mathrm{~m}$ by $4 \mathrm{~m}$ and $S=2$ radar modules with $M=1$ transmitters and $N=3$ receivers are placed outside the rooms. The region of interest is discretized into $P_{x} \times P_{y}=64 \times 64$ pixels. The three different scenarios with $R=7$ paths are also described in Section 4.5.4 and illustrated in Figures 4.16 and 4.18 .

Errors in positions of the front and back walls are not considered, since the multipath returns are assumed to be due to secondary reflections at the side walls only. The initial estimate of the locations of the side walls is drawn from a uniform distribution. The distribution is centered around the true wall locations and extends to errors of $\pm 0.5 \mathrm{~m}$. This error complies with the expected performance of building layout estimation techniques as mentioned earlier. The reconstruction utilizes the builtin Matlab implementation of a QN method and the particle swarm optimization toolbox for Matlab [Che14] for solving the outer non-convex optimization problem in the simulations. The QN method uses an active-set algorithm with finite-difference gradient evaluation. For PSO, 20 particles have been used over 100 generations. SparSA [WNF09] is used to solve the inner convex problem. The regularization parameter is chosen as $\lambda=\lambda_{\text {norm }}\left\|\breve{\boldsymbol{A}}(\boldsymbol{w})^{H} \breve{\boldsymbol{z}}\right\|_{\infty}$, with $\lambda_{\text {norm }}=0.3$.

Scenario A In Scenario A eight targets are imaged with two radar modules located at two opposing walls and located next to each other at the same wall, respectively. Refer to Figure 4.16 for the exact layout of the scene. Example reconstructions of the scene under various conditions are depicted in Figure 5.8. The reconstructions with known wall location, see Figures 5.8a,d are the same as before and are shown again for comparison. If the initial estimates of the side wall positions are set to $1.6 \mathrm{~m}$ and $-1.7 \mathrm{~m}$ with respect to the $\mathrm{y}$-axis of the coordinate system, image reconstruction fails completely for both distributed and co-located configurations, see Figures 5.8b,e. This illustrates the need for the proposed wall correction method. When applied to the considered scenario, the reconstruction results yield the images in Figures 5.8c,f. 


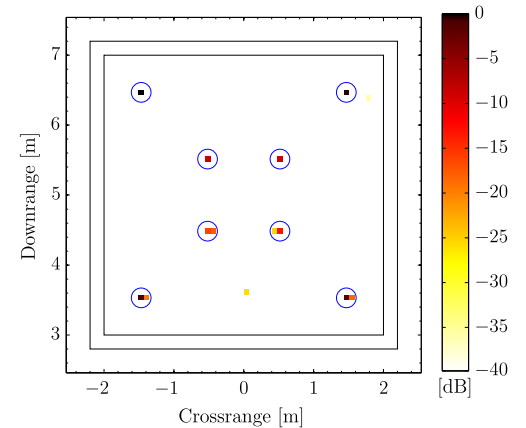

(a) Benchmark, distributed

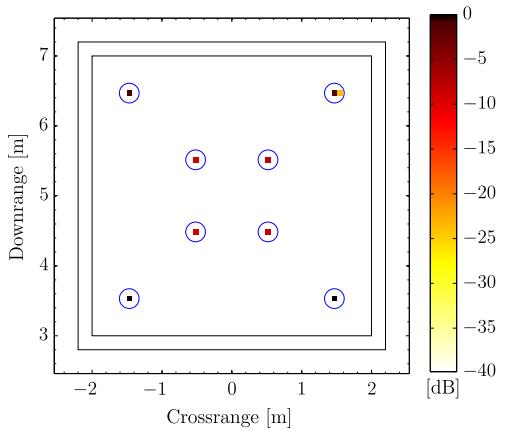

(d) Benchmark, co-located

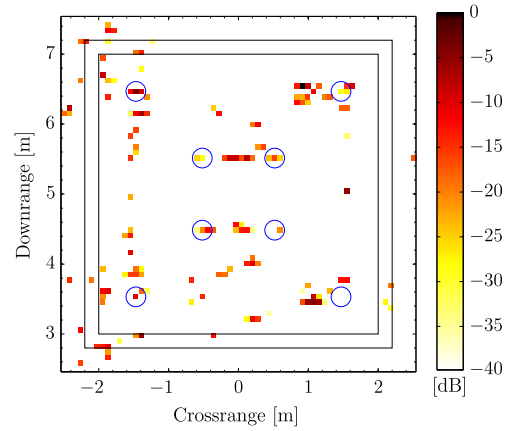

(b) Wall error, distributed

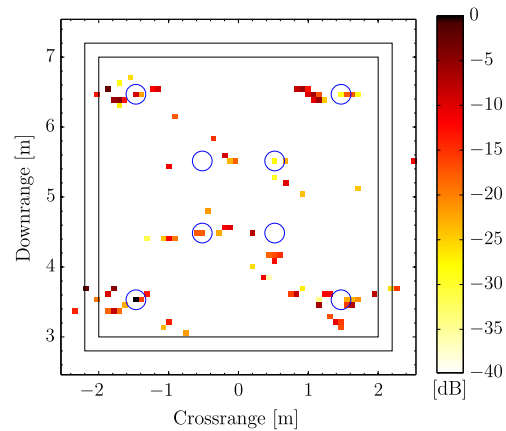

(e) Wall error, co-located

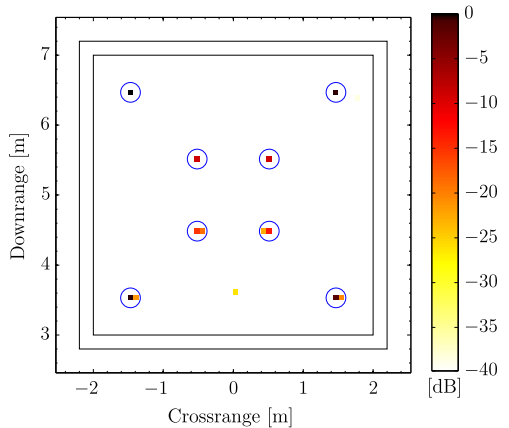

(c) PSO wall corr., distributed

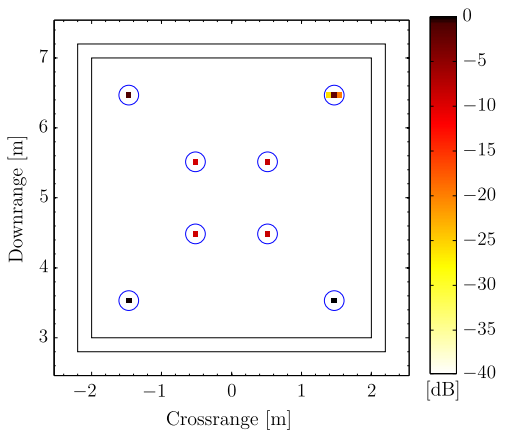

(f) PSO wall corr., co-located

Figure 5.8. Reconstruction results for Scenario A: Opposing walls versus same wall placement.

Evidently, the PSO approach is able to enhance the image quality, with the result being on par with the known wall location or benchmark case. The estimated wall positions are $1.999 \mathrm{~m}$ and $-2.003 \mathrm{~m}$ for the distributed case and $1.999 \mathrm{~m}$ and $-2.001 \mathrm{~m}$ for the co-located case. Hence, the estimation error is in the millimeter range for both configurations. The QN approach yields similar results.

Quantitative comparison of the reconstruction quality is achieved using the Earth Mover's Distance (EMD). The mean, median and standard deviation of the EMD for 50 Monte Carlo runs are listed in Table 5.1 for the various considered cases. Apparently, the distributed setup fails to reconstruct the scene reliably, as the error and the variance are relatively large not only for the wall location correction methods but also the benchmark case. In the co-located case, the wall correction methods almost achieve the same image quality as the benchmark reconstruction, where the PSO method is outperforming the QN method. 
Table 5.1. Scenario A: EMD comparison across 50 Monte Carlo runs.

\begin{tabular}{|c|c|c|c|c|}
\hline & $\begin{array}{l}\text { Wall error } \\
\text { dist co-loc }\end{array}$ & $\begin{array}{l}\text { Benchmark } \\
\text { dist co-loc }\end{array}$ & $\begin{array}{l}\text { QN corr. } \\
\text { dist co-loc }\end{array}$ & $\begin{array}{l}\text { PSO corr. } \\
\text { dist co-loc }\end{array}$ \\
\hline & 161.6961 .54 & $\begin{array}{ll}19.01 & 3.89\end{array}$ & $\begin{array}{ll}28.50 & 5.63\end{array}$ & $\begin{array}{ll}56.89 & 3.94\end{array}$ \\
\hline & .3357 & 13. & $11.03 \quad 3.77$ & $5.48 \quad 3.85$ \\
\hline itd. & $88.20 \quad 25.26$ & $69.75 \quad 0.48$ & $31.44 \quad 4.62$ & $\begin{array}{ll}337.68 & 0.50\end{array}$ \\
\hline
\end{tabular}

\section{Scenario B}

Scenario B compares co-located and distributed placements of two modules along the front wall without target shadowing. The scene layout is illustrated in Figure 4.18. Corresponding example reconstructions for the various considered cases are shown in Figure 5.9. As expected, the sparse reconstruction fails for erroneous wall locations, whereas the proposed wall location correction scheme based on the PSO method yields almost perfect images of the scene for both distributed and co-located cases. This performance is also reflected by the high accuracy of the wall locations estimates; $2.001 \mathrm{~m}$ and $-1.999 \mathrm{~m}$ for the distributed case and $1.998 \mathrm{~m}$ and $-2.000 \mathrm{~m}$ for the colocated case. The above qualitative observations are also confirmed by the quantitative EMD results summarized in Table 5.1. As expected from the theoretical dictionary analysis, the distributed case is slightly inferior to the co-located case. Note that for some noise realizations the reconstruction fails, leading to outliers in the EMD values. Hence, the median may give a better indication of the average or expected performance.

\section{Scenario C}

Scenario C is using the same layout as Scenario B, however, the targets in the front are assumed to block the direct propagation paths to the two targets in the back. The details of the shadowing have been described in Section 4.5.4.

Table 5.2. Scenario B: EMD comparison across 50 Monte Carlo runs.

\begin{tabular}{c|cc|cc|rr|rr} 
& \multicolumn{2}{|c|}{ Wall } & error & \multicolumn{2}{|c|}{ Benchmark } & \multicolumn{2}{|c|}{ QN corr. } & \multicolumn{2}{|c}{ PSO corr. } \\
& dist & co-loc & dist & co-loc & dist & co-loc & dist & co-loc \\
\hline mean & 51.98 & 36.50 & 1.64 & 1.39 & 5.57 & 1.62 & 4.98 & 1.42 \\
median & 45.31 & 36.55 & 1.59 & 1.41 & 1.95 & 1.42 & 1.56 & 1.40 \\
std. dev. & 28.71 & 19.51 & 0.42 & 0.24 & 5.72 & 0.94 & 17.45 & 0.34
\end{tabular}




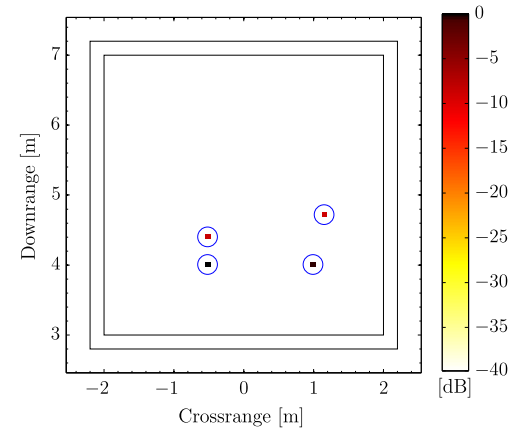

(a) Benchmark, distributed

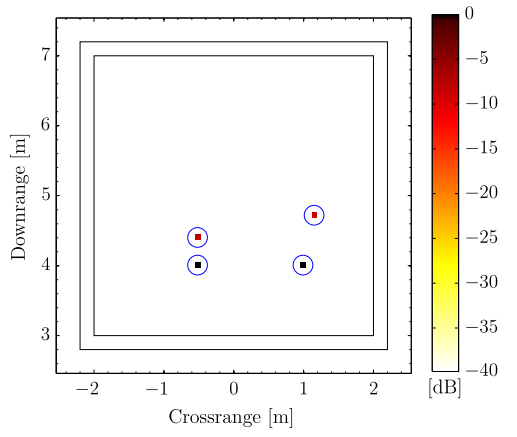

(d) Benchmark, co-located

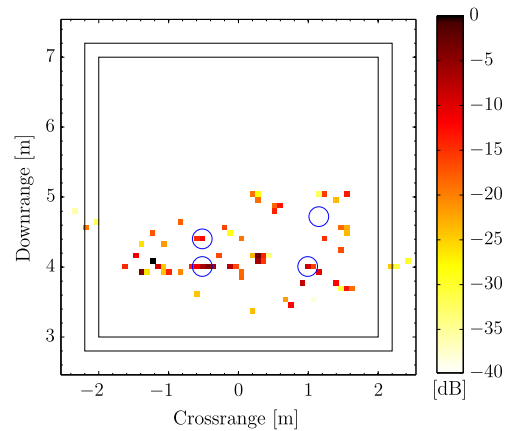

(b) Wall error, distributed

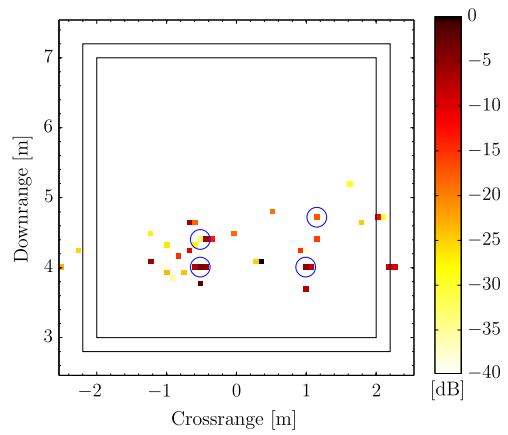

(e) Wall error, co-located

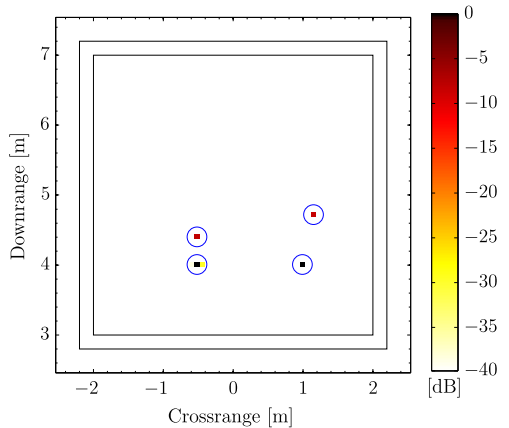

(c) PSO wall corr., distributed

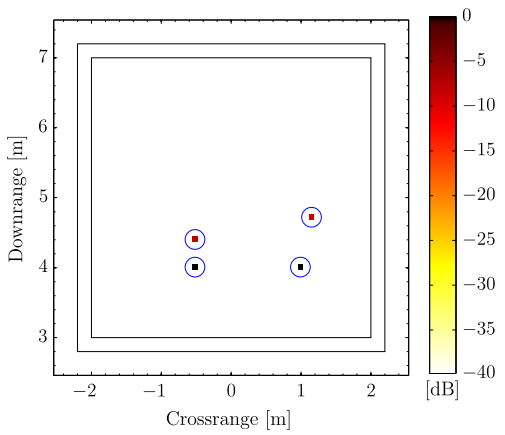

(f) PSO wall corr., co-located

Figure 5.9. Reconstruction results for Scenario B: Widely-spaced versus closely-spaced placement without shadowing.

Figure 5.10 shows the same selection of example results as in Figure 5.9. The colocated configuration shows weak reconstructions for both of the shadowed targets for the benchmark as well as the wall correction results, see Figures 5.10d,f. In contrast to that, the benchmark and wall corrected reconstructions for the distributed case in Figure 5.10a,c depict the shadowed targets about $6 \mathrm{~dB}$ stronger than in the colocated case. As in the previous examples, the sparse reconstruction without wall error correction, depicted in Figures 5.10b,e, fails. Furthermore, accurate wall location estimation results are obtained: $2.000 \mathrm{~m}$ and $-2.005 \mathrm{~m}$ for the distributed and $2.000 \mathrm{~m}$ and $-1.999 \mathrm{~m}$ for the co-located case.

These observations are confirmed by the EMD-based Monte Carlo results, summarized in Table 5.3. For known wall locations and PSO-based wall error correction, median reconstruction performance in the distributed case slightly outperforms a co-located placement of the units. The inferior performance of the distributed layout in the mean EMD is attributed to outliers in the reconstruction results. The rather small difference in the EMD between the distributed and co-located cases can be explained by the working principle of the metric. In case of a total intensity mismatch in the images, the difference in intensity is penalized. However, the difference is evaluated on a linear 


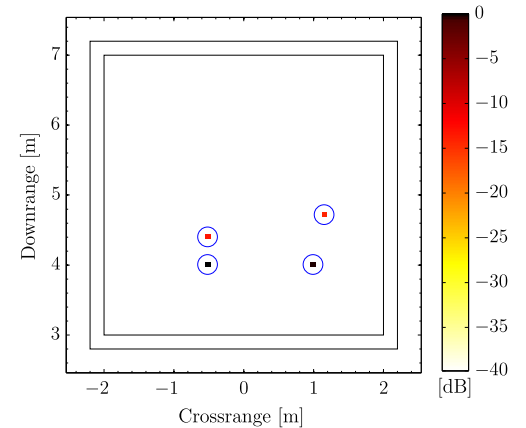

(a) Benchmark, distributed

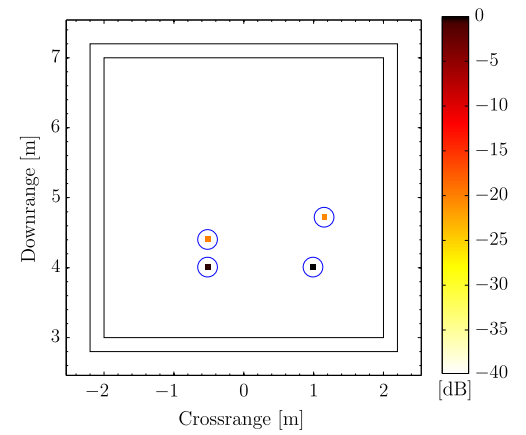

(d) Benchmark, co-located

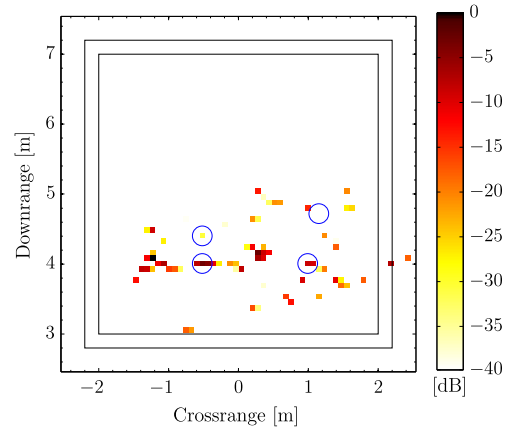

(b) Wall error, distributed

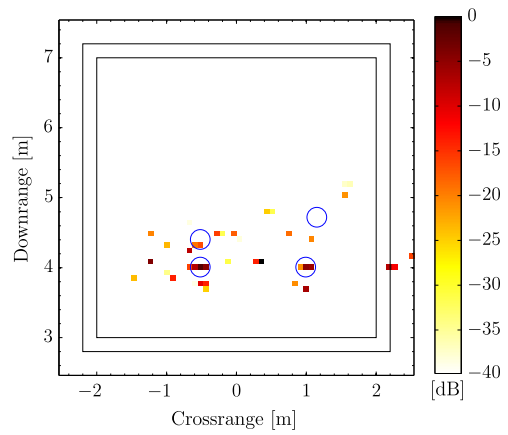

(e) Wall error, co-located

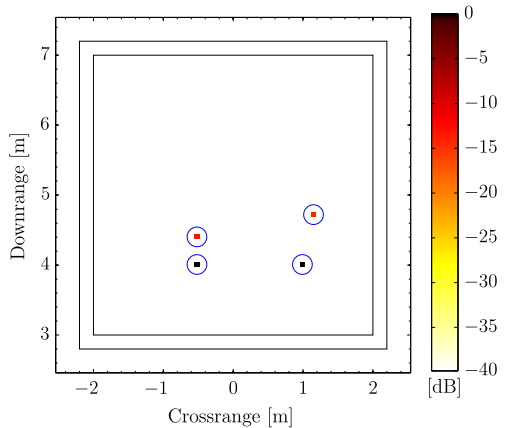

(c) PSO wall corr., distributed

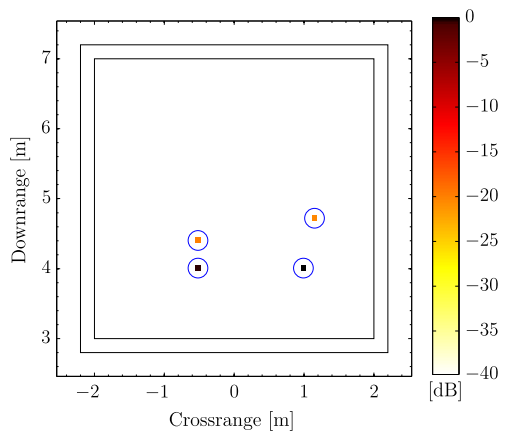

(f) PSO wall corr., co-located

Figure 5.10. Reconstruction results for Scenario C: Widely-spaced versus closely-spaced placement including shadowing.

Table 5.3. Scenario C: EMD comparison across 50 Monte Carlo runs.

\begin{tabular}{c|cc|cc|cc|cc} 
& \multicolumn{2}{|c|}{$\begin{array}{c}\text { Wall error } \\
\text { dist }\end{array}$} & \multicolumn{2}{|c|}{ co-loc } & \multicolumn{2}{|c|}{ dist } & co-loc & \multicolumn{2}{|c|}{ dist } & co-loc & \multicolumn{2}{c}{$\begin{array}{c}\text { PSO corr. } \\
\text { dist }\end{array}$} & co-loc \\
\hline mean & 45.08 & 35.01 & 4.16 & 3.97 & 7.28 & 4.07 & 3.22 & 3.97 \\
median & 41.78 & 31.83 & 3.10 & 3.96 & 3.34 & 3.98 & 3.11 & 3.98 \\
std. dev. & 23.26 & 21.85 & 7.15 & 0.26 & 9.07 & 0.59 & 0.59 & 0.25
\end{tabular}

amplitude scale. The reconstructed normalized intensity of the shadowed targets differs by less then 0.1, hence, the difference in the EMD is very low.

\section{Experimental Results}

The wideband real aperture pulse-Doppler radar with $M=1$ transmitter and a uniform linear array with $N=8$ receivers from the Radar Imaging Lab, Villanova University was again used to collect measurements. The transmitter and the array were placed on the same baseline with a lateral spacing of $29.2 \mathrm{~cm}$. The distance of the transmitter to a $0.3 \mathrm{~m}$ thick reinforced concrete side wall was $62 \mathrm{~cm}$. In total, $R=4$ propagation 


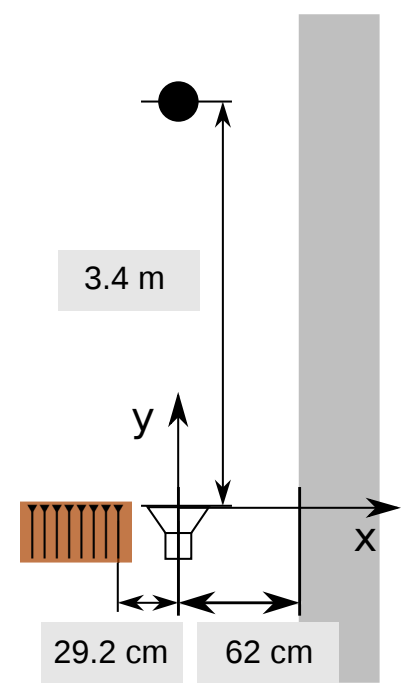

Figure 5.11. Scene geometry for the lab experiment.

paths were considered, namely, the direct path, two paths with a single reflection at the side wall and one with double reflection at the side wall. The scene consists of a single aluminum pipe placed at $3.4 \mathrm{~m}$ downrange directly in front of the transmitter. Refer to Figure 5.11 for an illustration of the scene setup.

As a reference, the benchmark result where the actual wall position has been used is shown in Figure 5.12a. The reconstruction result with an erroneous wall location is shown in Figure 5.12b, whereas the PSO-based wall correction result is shown in Figure 5.12c. The QN-based method yields similar results, as depicted in Figure 5.12d. The dashed lines represent the assumed and estimated wall locations in Figure 5.12b and Figures 5.12c,d, respectively. Similar to the simulation results, the reconstruction fails when the assumed wall location is incorrect. Using the proposed method, the wall location has been accurately estimated and the corresponding reconstruction yields an image on par with the benchmark.

\subsection{Conclusions}

In this chapter, two types of adverse wall effects have been addressed, namely, reflections from the front wall and uncertainties in the position of the interior walls. The front walls returns and wall reverberation introduce strong artifacts and render the scene less sparse. A method has been proposed that jointly models and reconstructs wall reflections and target returns. Thus, the wall returns can be removed from the image leading to a clearer representation of the scene of interest. Furthermore, it has 


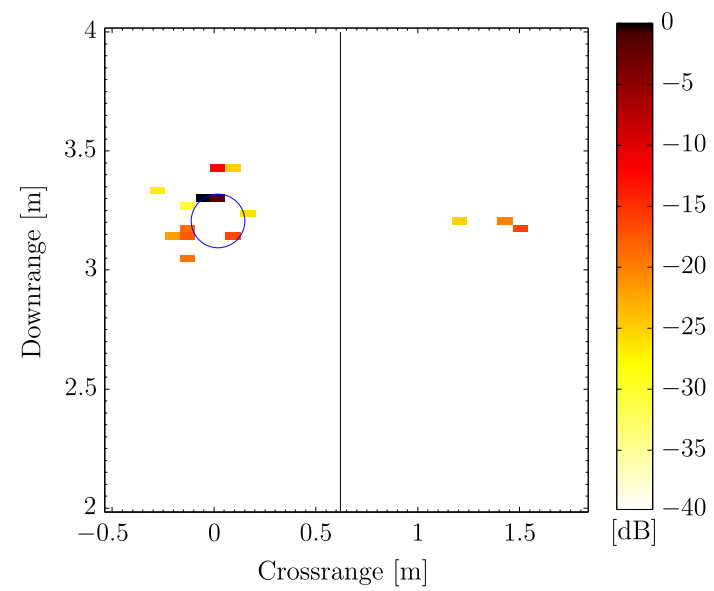

(a) Benchmark

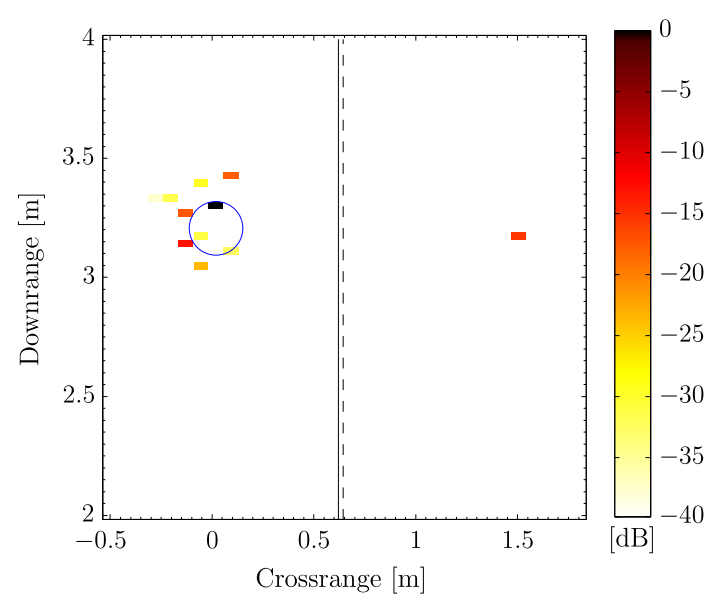

(c) PSO wall correction

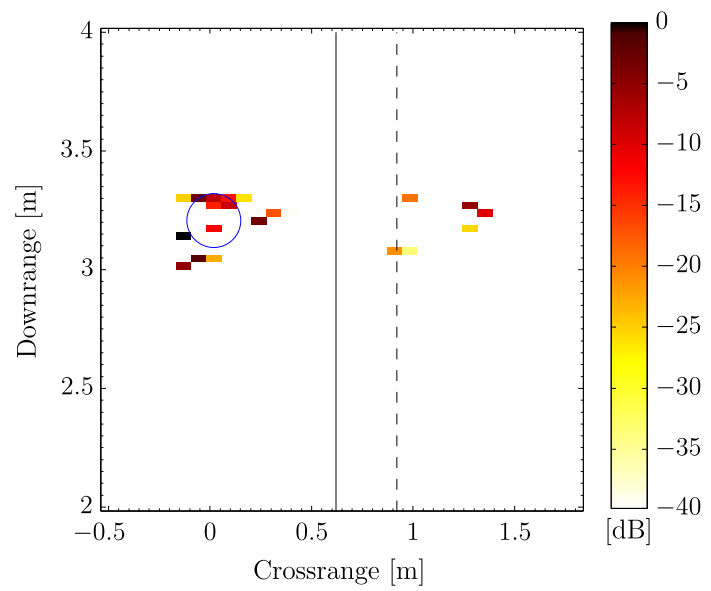

(b) Wall error

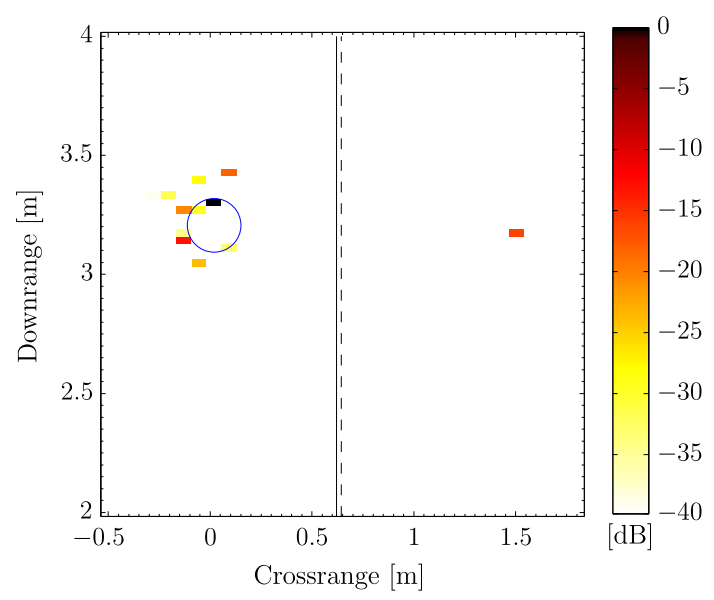

(d) QN wall correction

Figure 5.12. Reconstruction result using experimental data with one unknown side wall.

been shown that errors in the interior wall locations lead to failure of the previously proposed sparsity-based multipath exploitation method. To this end, the wall locations have been introduced as additional parameters that have to be estimated from the measurements. The proposed approach jointly estimates the wall locations and reconstructs the scene. This leads to good imaging performance in the case of limited prior knowledge of the wall locations. The effectiveness of the proposed methods has been shown using simulated and experimental data. 



\section{Chapter 6}

\section{Conclusions and Outlook}

In this thesis, the problem of multipath propagation in Through-the-Wall Radar Imaging (TWRI) has been considered from a sparse reconstruction perspective. Compressive Sensing (CS) allows for excellent imaging results in scenarios with limited measurements of the scene. Utilizing a ray-tracing model for the propagation of the electromagnetic waves, multipath has been exploited in the image formation. CS-based multipath exploitation methods have been proposed which yield highly-resolved and artifact-free images of stationary and moving targets. Adverse effects related to reflections from the building structure have been tackled using joint reconstruction approaches.

A summary of the work and the main conclusions are provided in Section 6.1. Section 6.2 gives an outlook regarding possible future work.

\subsection{Conclusions}

\subsubsection{Multipath Model}

A comprehensive received signal model has been introduced that considers stationary and moving targets, wall effects, and multipath propagation. Target and wall returns have been modeled individually to separate their effects. The target model includes all propagation paths that interact with the targets of interest along their way. Conversely, the wall model considers returns that solely interact with the fixed building structure. Both models consist of additive linear components, each of which corresponds to a particular path. A concise linear model is necessary for multipath exploitation via sparse reconstruction.

\subsubsection{Sparsity-Based Multipath Exploitation}

The problem of multipath exploitation for TWRI in view of sparsity-based reconstruction methods has been considered. The underlying idea is to take advantage of the 
additional energy and information on the targets. CS-based multipath exploitation approaches for image reconstruction have been proposed. Hereby, three cases have been investigated, namely, stationary targets, moving targets and distributed radar.

First, stationary targets and stepped-frequency radar measurements were assumed. The group sparse structure of the multipath images has been identified and was used in the reconstruction approach. Moreover, the method has been extended to moving targets and Ultra-Wideband (UWB) radar. Reconstructing targets in a locationvelocity space with four dimensions resulted in high computational complexity. Hence, a numerically efficient two-step scheme was developed that first localizes the targets using CS. In a second step, the velocities are estimated by exploiting the Doppler information in multipath. The notion of group sparsity has further been extended to the case of multiple radar modules. Analyses of the sensing matrices showed that a co-located placement is generally superior to a distributed configuration of the radar system. However, if targets are shadowed, a distributed radar configuration is less susceptible to the arising model mismatch.

The proposed methods have been assessed based on simulation and experimental results. Highly-resolved images were obtained which are completely free from multipath ghost artifacts. Especially for velocity estimation, multipath has been shown to be an advantage rather than a nuisance. For all discussed scenarios, efficient sensing and reconstruction allowed a significant reduction of the sampling requirements as compared to conventional image formation methods.

\subsubsection{Mitigating Wall Effects and Uncertainties}

Effects caused by the building walls have been identified as a challenging issue in scene reconstruction. The strong returns from the front wall had to be suppressed to avoid excessive clutter in the image of the targets. A sparsity-based joint wall and target image reconstruction confined wall clutter to the wall image leading to an artifact-free target image.

Uncertainties in the building layout severely affected the performance of multipath exploitation. Thus, the signal model has been extended to include the unknown positions of the interior walls. This nonlinear model was employed in a CS-based joint wall parameter estimation and image reconstruction approach. The method allows for multipath exploitation in scenes with limited knowledge of the building layout. 


\subsection{Outlook}

\subsubsection{Signal Model}

In this thesis, the propagation model has been restricted to the intuitive Geometrical Optics (GO) approximation. Using a more sophisticated approach, the wave properties could be captured resulting in a more accurate description of multipath [GS13, GS14, GRSS14]. Furthermore, propagation losses and antenna patterns have been neglected. These could be readily included in the received signal model (3.21).

Targets have been assumed point-like and frequency independent. While including frequency-dependency of the targets is relatively straight-forward, dealing with extended targets is more challenging. First, the assumption of independent scattering centers as used in GO is violated. Second, the reflections occur at different points of the target when observed via various paths. In effect, the images associated with the various paths do not perfectly overlap anymore. Hence, a novel concept of approximate group sparsity would be needed to account for this effect.

Finally, the geometry of the building been restricted to a single room with rectangular shape. A realistic building consists of several rooms resulting in additional interior walls and corners. A more complex, building layout model needs to be developed to apply the proposed methods in this case.

\subsubsection{Sparsity-Based Multipath Exploitation}

Prior knowledge of the number of propagation paths and their topology has been assumed for multipath exploitation. Model selection approaches may be used to choose the correct number and types of multipath from a candidate set. Information theoretic criteria, such as the Akaike information criterion [Aka74] could be used for model order selection to avoid overfitting.

In the multiple radar module configuration, a centralized scheme has been used where all measurements are available at a single processing node. For practical purposes, a decentralized approach would be beneficial. Each of the modules carries out part of the reconstruction while communicating with the other units. This results in simpler and more flexible operation of the TWRI system and avoids a single point of failure. Proper algorithm design should ensure that the reconstruction quality is on par with 
centralized processing. Limits on computational complexity as well as communication overhead has to be considered in this case. Preliminary results regarding this idea have been published in $\left[\mathrm{SLA}^{+} 15\right]$.

This work considered the reconstruction of an accurate image of the scene of interest to be the final result. Practical systems would benefit from a subsequent automatic detection and classification to reduce the work load for a human operator. Existing work considers detection and feature extraction based on conventionally beamformed images [Deb10]. However, the image and feature statistics for a CS reconstruction result is vastly different. Approaches should be developed that enable target classification while taking CS into account.

The choice of the regularization parameter $\lambda$ is also an open problem that has not been addressed in this thesis. Various heuristic methods exist [CDS01, BDB07, GMS09b]. An extensive study should aim for an adapted criterion for TWRI purposes. Ideally, the choice should not rely on any prior knowledge of the scene or the nature of the noise. Rather, the selection should be based on the measurements and the reconstruction result.

\subsubsection{Sparse Reconstruction With Parameter Uncertainties}

The proposed wall position estimation and image reconstruction method has been restricted to the side wall locations only. The approach allows for extension to other parameters. The thickness and permittivity of the front wall can be included to provide auto-focusing through dielectric slabs. Similarly, periodically structured walls such as cinder block walls or reinforced concrete may be modeled. In a distributed scenario, the exact locations and orientations of the radar modules may not be known. This results in a calibration problem in order to align the measurements and images from the various units. The unknown calibration parameters could be obtained using the proposed joint estimation and reconstruction method.

The above mentioned approach includes a non-linear optimization over the set of unknown parameters. Particle Swarm Optimization (PSO) was shown to be effective, however, the computational burden was very high. Especially, when additional unknown parameters are included in the problem, the proposed approach becomes quickly intractable. More efficient methods are desirable to solve the non-linear optimization problem. In many cases reconstruction accuracy may be traded in for computational efficiency. 


\section{Appendix}

\section{A.1 Complex Amplitude Derivation}

For each path, the complex amplitude $\Gamma_{p m n}^{\left(\mathcal{P}_{r}\right)}$ can be derived from the dielectric properties of the front and interior walls and the corresponding angles of incidence and refraction. A path $\mathcal{P}_{r}$ consists of two partial paths, $\mathcal{P}_{r_{1}}^{\prime}$ and $\mathcal{P}_{r_{2}}^{\prime \prime}$, describing the propagation from the transceiver to the target and from the target back to the transceiver, respectively. Therefore, the complex amplitude associated with the total path equals the product of the complex amplitudes of the two partial paths, each consisting of one transmission coefficient associated with the front wall and one reflection coefficient, resulting in [Bal89]

$$
\Gamma_{p m n}^{\left(\mathcal{P}_{r}\right)}=\Gamma_{p m}^{\left(\mathcal{P}_{r_{1}}^{\prime}\right)} \Gamma_{p n}^{\left(\mathcal{P}_{r_{2}}^{\prime \prime}\right)}=\Upsilon^{\left(\mathcal{P}_{r_{1}}^{\prime}\right)} \Lambda^{\left(\mathcal{P}_{r_{1}}^{\prime}\right)} \Lambda^{\left(\mathcal{P}_{r_{2}}^{\prime \prime}\right)} \Upsilon^{\left(\mathcal{P}_{r_{2}}^{\prime \prime}\right)}
$$

The following equations hold for vertical polarization. Similar expressions can be found for the horizontally polarized case. The reflection coefficient $\Lambda^{(\cdot)}$, associated with a particular partial path, is given by [Bal89]

$$
\Lambda^{(\cdot)}= \begin{cases}\frac{\cos \theta_{\mathrm{i}, p m n}^{(\cdot)}-\sqrt{\varepsilon_{\mathrm{r}}} \cos \theta_{\mathrm{t}, p m n}^{(\cdot)}}{\cos \theta_{\mathrm{i}, p m n}^{(\cdot)}+\sqrt{\varepsilon_{\mathrm{r}}} \cos \theta_{\mathrm{t}, p m n}^{(\cdot)}}, & \text { for multipath via interior wall } \\ 1, & \text { otherwise. }\end{cases}
$$

Further, $\Upsilon^{(\cdot)}$ is the total transmission coefficient for a wave traveling through the front wall. The refraction on the first and second interface, respectively, and $b$ reverberations within the wall are considered [Bal89]

$$
\begin{aligned}
\Upsilon^{(\cdot)}= & \frac{2 \cos \theta_{\mathrm{i}, p m n}^{(\cdot)}}{\cos \theta_{\mathrm{i}, p m n}^{(\cdot)}+\sqrt{\varepsilon_{\mathrm{r}}} \cos \theta_{\mathrm{t}, p m n}^{(\cdot)}} \frac{2 \sqrt{\varepsilon_{\mathrm{r}}} \cos \theta_{\mathrm{t}, p m n}^{(\cdot)}}{\cos \theta_{\mathrm{i}, p m n}^{(\cdot)}+\sqrt{\varepsilon_{\mathrm{r}}} \cos \theta_{\mathrm{t}, p m n}^{(\cdot)}} \\
& \times\left(\frac{-\cos \theta_{\mathrm{i}, p m n}^{(\cdot)}+\sqrt{\varepsilon_{\mathrm{r}}} \cos \theta_{\mathrm{t}, p m n}^{(\cdot)}}{\cos \theta_{\mathrm{i}, p m n}^{(\cdot)}+\sqrt{\varepsilon_{\mathrm{r}}} \cos \theta_{\mathrm{t}, p m n}^{(\cdot)}}\right)^{2 b} .
\end{aligned}
$$

In all of the above equations, $\theta_{\mathrm{i}, p m n}^{(\cdot)}$ and $\theta_{\mathrm{t}, p m n}^{(\cdot)}$ are the incident (in air) and refracted (in the medium) angles of the wave and $\varepsilon_{\mathrm{r}}$ is the relative permittivity of the interior wall. In the case of wall ringing multipath $b$ is larger then zero, otherwise it is zero. A more detailed derivation of the path loss coefficients can be found in [SAA11]. 


\section{A.2 Justification of the Invariance of Complex Am- plitude Across the Array}

In the derivation of the multipath exploitation scheme, the complex path weights are assumed invariant across the array elements and, therefore, can be replaced by a common weight. This approximation generally holds for far-field conditions, where all angles are approximately equal across all target/array element pairs. The approximation also holds to a certain extent for the near-field case. To demonstrate this property, the error for monostatic near-field imaging is examined. The array length is chosen as $1.5 \mathrm{~m}$ and the imaged region is within a $4 \mathrm{~m}$ by $5 \mathrm{~m}$ room, whose center is at $4.5 \mathrm{~m}$ downrange. For the whole image grid, the individual path loss coefficients associated with a propagation path $\mathcal{P}_{r}$ are calculated according to (A.1). Subsequently, the relative errors are calculated for every target, path and array element. The relative error is defined as

$$
\xi_{p m n}^{\left(\mathcal{P}_{r}\right)}=\frac{\left|\Gamma_{p}^{\left(\mathcal{P}_{r}\right)}-\Gamma_{p m n}^{\left(\mathcal{P}_{r}\right)}\right|}{\left|\Gamma_{p m n}^{\left(\mathcal{P}_{r}\right)}\right|},
$$

where $m=0, \ldots, M-1, n=0, \ldots, N-1, p=0, \ldots, P-1, r=0, \ldots, R-1$. The common amplitude factors $\Gamma_{p}^{\left(\mathcal{P}_{r}\right)}$ are chosen as the mean,

$$
\Gamma_{p}^{\left(\mathcal{P}_{r}\right)}=\frac{1}{M N} \sum_{m=0}^{M-1} \sum_{n=0}^{N-1} \Gamma_{p m n}^{\left(\mathcal{P}_{r}\right)}, \quad p=0, \ldots, P-1, r=0, \ldots, R-1 .
$$

A relative error threshold of $10 \%$ is assumed acceptable. The ratio of relative errors meeting this criterion is calculated, i.e., $\#\left\{\xi_{p m n}^{\left(\mathcal{P}_{r}\right)}<0.1, m=0, \ldots, M-1, n=0, \ldots, N-\right.$ $1, p=0, \ldots, P-1\} /(M N P)$.

For the multipath associated with the back wall and the back right corner, the error of using $\Gamma_{p}^{\left(\mathcal{P}_{r}\right)}$ instead of $\Gamma_{p m n}^{\left(\mathcal{P}_{r}\right)}$ is sufficiently low for all cases. Hence, no significant errors are experienced when using the above assumption. However, the approximation is less accurate when considering the multipath via the left side wall. For these paths, due to the higher variation in incident and reflection angles, only $90 \%$ of the approximation errors stay below the threshold. This approximation error is still comparably low and will probably not affect the performance of the multipath exploitation scheme in nearfield scenarios. Note that the reflection coefficients are purely real for perfect dielectric slabs. This alleviates the problem further, as the beamformer is less susceptible to amplitude errors as compared to phase errors. 


\section{List of Acronyms}

BOMP Block Orthogonal Matching Pursuit

BP Basis Pursuit

BPDN Basis Pursuit De-Noising

CoSaMP Compressive Sampling Matching Pursuit

CPI Coherent Processing Interval

CS Compressive Sensing

DFT Discrete Fourier Transform

DP direct path

DSBF Delay-and-Sum Beamforming

EM Electro-Magnetic

EMD Earth Mover's Distance

FD Frequency Domain

FoGLasso Fast overlapping Group Lasso

GO Geometrical Optics

GPR Ground Penetrating Radar

GS Group Sparse

GSCS Group Sparse Compressive Sensing

i.i.d. independent and identically distributed

MP multipath

NFFT Non-Equispaced Fast Fourier Transform

NSP Null Space Property

OMP Orthogonal Matching Pursuit

PRI Pulse Repetition Interval

PSO Particle Swarm Optimization 
QN Quasi-Newton

RCS Radar Cross Section

RF Radio Frequency

RIP Restricted Isometry Property

RMSE Root Mean Squared Error

ROC Receiver Operating Characteristic

SAR Synthetic Aperture Radar

SCR Signal-to-Clutter Ratio

SNR Signal-to-Noise Ratio

SP Subspace Pursuit

SparSA Sparse Reconstruction by Separable Approximation

TWRI Through-the-Wall Radar Imaging

UUP Uniform Uncertainty Principle

UWB Ultra-Wideband 


\section{List of Symbols}

\section{Symbols}

$\boldsymbol{a}_{i} \quad i$-th column of the sensing matrix

$\boldsymbol{A} \quad$ Sensing matrix

$\boldsymbol{A}_{i} \quad i$-th block of a block-structured sensing matrix

$\boldsymbol{A}_{\text {distr }} \quad$ Sensing matrix for distributed radar

$\breve{A} \quad$ Sensing matrix for multiple radar modules

$b \quad$ Index variable for wall ringing

$B \quad$ Number of wall ringing responses

c Speed of light

d Block size in block-sparsity

$d_{\text {wall }} \quad$ Thickness of front wall

f Frequency variable

$f_{c} \quad$ Center frequency

$f_{s} \quad$ Sampling rate

$g_{p} \quad$ Index set for $p$-th group in target image

$h_{p} \quad$ Index set for $p$-th group in wall image

$I_{p} \quad$ Delay-and-Sum Beamforming (DSBF) image at $p$-th pixel

$I_{p}(k) \quad$ DSBF image at $p$-th pixel for $k$-th pulse

$J$

Total number of measurements after downsampling

$k \quad$ Index variable for slow time or pulses

$K \quad$ Number of pulses in slow time

$K_{\mathrm{d}} \quad$ Number of pulses in slow time after downsampling

$l \quad$ Index variable for frequency bins

$L \quad$ Number of frequency bins in stepped-frequency radar

$L_{u} \quad$ Length of $u$-th corner

$m \quad$ Index variable for transmitters

$M \quad$ Number of transmitters

$M_{\mathrm{d}} \quad$ Number of active transmitters after downsampling

$\boldsymbol{M}^{(r)} \quad$ Masking matrix for wall model corresponding to $r$-th path

$n \quad$ Index variable for receivers

$N \quad$ Number of receivers

$N_{\mathrm{d}} \quad$ Number of active receivers after downsampling 


\begin{tabular}{|c|c|}
\hline$N_{\mathrm{c}}$ & Number of corners \\
\hline$n$ & Measurement noise vector \\
\hline$\tilde{n}$ & Measurement noise vector for multiple radar modules \\
\hline$p$ & $\begin{array}{l}\text { Index variable for image pixel (location only) or target states (lo- } \\
\text { cation and velocity) }\end{array}$ \\
\hline$P$ & Number of image pixels or target states \\
\hline$P_{v}$ & Number of velocity bins \\
\hline$P_{x}$ & Number of pixels in crossrange \\
\hline$P_{y}$ & Number of pixels in downrange \\
\hline $\mathcal{P}, \mathcal{P}_{r}$ & $\begin{array}{l}\text { Round-trip path from a transmitter to a target and back to a re- } \\
\text { ceiver, via } r \text {-th path }\end{array}$ \\
\hline $\mathcal{P}^{\prime}, \mathcal{P}_{r}^{\prime}$ & One-way path from a target to a receiver, via $r$-th path \\
\hline $\mathcal{P}^{\prime \prime}, \mathcal{P}_{r}^{\prime \prime}$ & One-way path from a transmitter to a target, via $r$-th path \\
\hline$\tilde{\mathcal{P}}^{\prime}$ & Equivalent one-way path using a virtual target \\
\hline$r$ & Index variable for propagation paths \\
\hline$R$ & Number of propagation paths \\
\hline$R_{\mathrm{w}}$ & Number of propagation paths for wall model \\
\hline$r$ & General received signal vector \\
\hline$s(t)$ & Transmitted pulse in complex base-band \\
\hline$s^{\mathrm{Rx}}$ & Index variable of receiving radar module \\
\hline$s^{\mathrm{Tx}}$ & Index variable of transmitting radar module \\
\hline$S$ & Number of radar modules \\
\hline$s$ & General sparse vector \\
\hline$t$ & Continuous-time variable \\
\hline$T$ & Number of samples in fast-time \\
\hline$T_{\mathrm{d}}$ & Number of samples in fast-time after downsampling \\
\hline$T_{p}$ & Wideband pulse duration \\
\hline$T_{s}$ & Sampling interval \\
\hline$u$ & Index variable for corners \\
\hline$v_{D, p}^{(r)}$ & Apparent Doppler speed corresponding to $p$-th target and $r$-th path \\
\hline$v_{x p}$ & Velocity component in crossrange direction of the $p$-th target \\
\hline$v_{y p}$ & Velocity component in downrange direction of the $p$-th target \\
\hline$w$ & Vector of wall positions \\
\hline $\boldsymbol{W}^{(p)}, \boldsymbol{W}_{\mathrm{t}}^{(p)}$ & Weight matrix for $p$-th group in target image \\
\hline $\boldsymbol{W}_{\mathrm{w}}^{(p)}$ & Weight matrix for $p$-th group in wall image \\
\hline$x$ & Crossrange spatial variable \\
\hline
\end{tabular}


$x_{p}$

$\boldsymbol{x}_{p}$

$x$

$x_{p}$

$z_{m n k}(t)$

$z$

$\boldsymbol{z}_{m n k}$

$\boldsymbol{z}_{\mathrm{FD}}$

$\bar{z}$

$\overline{\boldsymbol{z}}_{\mathrm{FD}}$

$\overline{\boldsymbol{z}}_{\mathrm{SP}}(k)$

$\breve{z}$

$\beta$

$\varepsilon_{\mathrm{r}}$

$\theta_{\text {air }}$

$\theta_{\text {wall }}$

$\lambda$

$\lambda_{\text {norm }}$

$\boldsymbol{\sigma}, \boldsymbol{\sigma}_{\mathrm{t}}$

$\boldsymbol{\sigma}^{(r)}, \boldsymbol{\sigma}_{\mathrm{t}}^{(r)}$

$\boldsymbol{\sigma}_{\mathrm{W}}$

$\boldsymbol{\sigma}_{\mathrm{W}}^{(r)}$

$\tilde{\boldsymbol{\sigma}}$

$\tilde{\boldsymbol{\sigma}}_{s^{\mathrm{Tx}}} s^{\mathrm{Rx}}$

$\tilde{\boldsymbol{\sigma}}_{\mathrm{j}}$

$\breve{\sigma}$

$\tau_{p m n}$

$\tau_{p m n}^{(r)}$

$\tau_{p m n}^{(r)}(k)$

$\phi^{(i)}$
Crossrange coordinate of the $p$-th target

Location of the $p$-th target

Downrange spatial variable

Downrange coordinate of the $p$-th target

Received signal corresponding to $m$-th transmitter, $n$-th receiver, and $k$-th pulse

Measurement vector

Measurement sub-vector corresponding to $m$-th transmitter, $n$-th receiver, and $k$-th pulse

Measurement vector in frequency domain

Undersampled measurement vector

Undersampled measurement vector in frequency domain

Undersampled measurement vector for $k$-th pulse

Measurement vector for multiple radar modules

Sparsity level, i.e., number of non-zeros

Relative permittivity of front wall

Angle of incidence/refraction in air

Angle of incidence/refraction in wall medium

Regularization parameter

Normalized regularization parameter

Vectorized target image

Vectorized target image corresponding to $r$-th path

Vectorized wall image

Vectorized wall image corresponding to $r$-th path

Vectorized and stacked target image for all paths

Vectorized and stacked target image for all paths when module $s^{\mathrm{Tx}}$ transmits and module $s^{\mathrm{Rx}}$ receives

Vectorized and stacked target and wall images for all paths

Vectorized and stacked target images for all paths and multiple radar modules

Round-trip propagation delay corresponding to $p$-th target, $m$-th transmitter and $n$-th receiver via direct path

Round-trip propagation delay corresponding to $p$-th target, $m$-th transmitter and $n$-th receiver via $r$-th path

Round-trip propagation delay corresponding to $p$-th target, $m$-th transmitter and $n$-th receiver via $r$-th path at pulse index $k$

$i$-th row of the measurement matrix 


\begin{tabular}{|c|c|}
\hline$\Phi$ & Measurement matrix \\
\hline$\Phi_{s}^{\mathrm{Rx}}$ & Measurement matrix corresponding to the receivers of module $s$ \\
\hline$\Phi_{s}^{\mathrm{Tx}}$ & $\begin{array}{l}\text { Measurement matrix corresponding to the transmitters of module } \\
s\end{array}$ \\
\hline $\mathrm{FD}$ & Measurement matrix for stepped-frequency radar \\
\hline SP & Measurement matrix for a single pulse \\
\hline & Dictionary matrix \\
\hline$\Psi^{(r)}$ & Dictionary matrix corresponding to $r$-th path \\
\hline$m n k$ & $\begin{array}{l}\text { Dictionary sub-matrix corresponding to } m \text {-th transmitter, } n \text {-th re- } \\
\text { ceiver, and } k \text {-th pulse }\end{array}$ \\
\hline$\Psi_{\mathrm{FD}}$ & Dictionary matrix for stepped-frequency radar \\
\hline$\Psi_{\mathrm{FD}}^{(r)}$ & $\begin{array}{l}\text { Dictionary matrix for stepped-frequency radar corresponding to } r \text { - } \\
\text { th path }\end{array}$ \\
\hline & Dictionary matrix for walls corresponding to $r$-th path \\
\hline$\tilde{\mathbf{\Psi}}, \tilde{\boldsymbol{\Psi}}(\boldsymbol{w})$ & $\begin{array}{l}\text { Concatenated dictionary matrix for all paths, depending on wall } \\
\text { positions }\end{array}$ \\
\hline $\mathrm{Tx}_{9} \mathrm{Rx}$ & $\begin{array}{l}\text { Concatenated dictionary matrix for all paths when module } s^{\mathrm{Tx}} \\
\text { transmits and module } s^{\mathrm{Rx}} \text { receives }\end{array}$ \\
\hline & $\begin{array}{l}\text { Concatenated dictionary matrix for all paths for stepped-frequency } \\
\text { radar }\end{array}$ \\
\hline
\end{tabular}

\section{Functions and Operators}

$\begin{array}{ll}\otimes & \text { Kronecker product } \\ \circ & \text { Element-wise (or Schur) product } \\ \mathbb{C} & \text { Set of complex numbers } \\ \mathbb{N} & \text { Set of natural numbers } \\ \mathbb{R} & \text { Set of real numbers } \\ \Re\{\cdot\} & \text { Real part } \\ (\cdot)^{T} & \text { Transpose of a vector or matrix } \\ (\cdot)^{H} & \text { Conjugate transpose of a vector or matrix } \\ (\cdot)^{*} & \text { Complex conjugate of a scalar, vector, or matrix } \\ (\cdot)^{+} & \text {Pseudoinverse of a vector or matrix } \\ (\cdot)^{-1} & \text { Inverse of a square matrix } \\ (\cdot) & \text { Estimate of a quantity } \\ |\cdot| & \text { Absolute value of a scalar }\end{array}$


$\lfloor\cdot\rfloor \quad$ Floor operator, i.e., rounding towards smallest integer

$\|\cdot\|_{0} \quad \ell_{0}$-pseudo-norm of a vector, i.e., number of non-zero elements

$\|\cdot\|_{1} \quad \ell_{1}$-norm of a vector

$\|\cdot\|_{2} \quad$ Euclidean norm or $\ell_{2}$-norm of a vector

$\|\cdot\|_{2,1} \quad$ Mixed $\ell_{2} / \ell_{1}$-norm of a vector, i.e., an $\ell_{1}$-norm of groups of $\ell_{2}$-norms

$\|\cdot\|_{\infty} \quad$ Maximum-norm of a vector

$\operatorname{diag}(\cdot) \quad$ Diagonal matrix created from argument

blkdiag $(\cdot) \quad$ Block diagonal matrix created from argument

$\boldsymbol{I}_{N} \quad$ Identity matrix of size $N$

mod Modulo operator

$\mu(\cdot) \quad$ Mutual coherence of a matrix

$\mu_{\mathrm{B}}(\cdot) \quad$ Block-coherence of a matrix

$\rho(\cdot) \quad$ Spectral norm of a matrix, i.e., the maximum singular value 



\section{Bibliography}

[AA08a] F. Ahmad and M. G. Amin, "Multi-location wideband synthetic aperture imaging for urban sensing applications," Journal of the Franklin Institute, vol. 345, no. 6, pp. 618-639, Sep. 2008.

[AA08b] M. G. Amin and F. Ahmad, "Wideband synthetic aperture beamforming for through-the-wall imaging [lecture notes]," IEEE Signal Processing Magazine, vol. 25, no. 4, pp. 110-113, Jul. 2008.

[AA12a] F. Ahmad and M. G. Amin, "Partially sparse reconstruction of behindthe-wall scenes," in Proceedings SPIE Symposium on Defense, Security, and Sensing, Compressive Sensing Conference, vol. 8365, Baltimore, USA, Apr. 2012.

[AA12b] F. Ahmad and M. G. Amin, "Wall clutter mitigation for MIMO radar configurations in urban sensing," in 11th International Conference on Information Science, Signal Processing and their Applications (ISSPA), Montreal, Canada, Jul. 2012, pp. 1165-1170.

[AA13] F. Ahmad and M. G. Amin, "Through-the-wall human motion indication using sparsity-driven change detection," IEEE Transactions on Geoscience and Remote Sensing, vol. 51, no. 2, pp. 881-890, Feb. 2013.

[AAK05a] F. Ahmad, M. G. Amin, and S. A. Kassam, "Synthetic aperture beamformer for imaging through a dielectric wall," IEEE Transactions on Aerospace and Electronic Systems, vol. 41, no. 1, pp. 271-283, 2005.

[AAK05b] F. Ahmad, M. G. Amin, and S. Kassam, "A beamforming approach to stepped-frequency synthetic aperture through-the-wall radar imaging," in IEEE Int. Workshop on Computational Advances in Multi-Sensor Adaptive Processing, Puerto Vallarta, Mexico, Dec. 2005, pp. 24-27.

[AAZ11] M. G. Amin, F. Ahmad, and W. Zhang, "A compressive sensing approach to moving target indication for urban sensing," in IEEE Radar Conference (RADAR), Kansas City, USA, May 2011, pp. 509-512.

[AFKA04] F. Ahmad, G. Frazer, S. Kassam, and M. G. Amin, "Design and implementation of near-field, wideband synthetic aperture beamformers," IEEE Transactions on Aerospace and Electronic Systems, vol. 40, no. 1, pp. 206220, Jan. 2004.

[Aka74] H. Akaike, "A new look at the statistical model identification," IEEE Transactions on Automatic Control, vol. 19, no. 6, pp. 716-723, 1974.

[Ami11] M. G. Amin, Ed., Through-the-Wall Radar Imaging. Boca Raton, FL: CRC Press, 2011.

[Ami15] M. G. Amin, Ed., Compressive Sensing for Urban Radar. Boca Raton, FL: CRC Press, 2015. 
[AQA15] F. Ahmad, J. Qian, and M. G. Amin, "Wall clutter mitigation using discrete prolate spheroidal sequences for sparse reconstruction of indoor stationary scenes," IEEE Transactions on Geoscience and Remote Sensing, vol. 53, no. 3, pp. 1549-1557, Mar. 2015.

[Bal89] C. Balanis, Advanced Engineering Electromagnetics. New York, NY: John Wiley and Sons, 1989.

[Bar06] E. Baranoski, "Visibuilding: Sensing through walls," in IEEE Workshop on Sensor Array and Multichannel Processing (SAM), Waltham, USA, Jul. 2006, pp. 1-22.

[Bar08] E. Baranoski, "Through-wall imaging: Historical perspective and future directions," Journal of the Franklin Institute, vol. 345, no. 6, pp. 556-569, Sep. 2008.

[BBC11] S. Becker, J. Bobin, and E. Candès, "NESTA: A fast and accurate firstorder method for sparse recovery," SIAM Journal on Imaging Sciences, vol. 4, no. 1, pp. 1-39, Jan. 2011.

[BCDH10] R. Baraniuk, V. Cevher, M. Duarte, and C. Hegde, "Model-based compressive sensing," IEEE Transactions on Information Theory, vol. 56, pp. 1982-2001, Apr. 2010.

[BCNV08] R. Baraniuk, E. Candès, R. Nowak, and M. Vetterli, "Compressive sampling [from the guest editors]," IEEE Signal Processing Magazine, vol. 25, no. 2, pp. 12-13, 2008.

[BDB07] P. Boufounos, M. Duarte, and R. Baraniuk, "Sparse signal reconstruction from noisy compressive measurements using cross validation," in IEEE/SP Workshop on Statistical Signal Processing (SSP), Madison, WI, Aug. 2007, pp. 299-303.

[Bey95] G. Beylkin, "On the fast Fourier transform of functions with singularities," Applied and Computational Harmonic Analysis, vol. 2, no. 4, pp. 363-381, 1995.

[BLZ12] M. Balthasar, M. Leigsnering, and A. Zoubir, "Compressive classification for through-the-wall radar imaging," in European Signal Processing Conference (EUSIPCO), Bucharest, Romania, Aug. 2012, pp. 2288-2292.

[Bur06] H. Burchett, "Advances in through wall radar for search, rescue, and security applications," in IET Conference on Crime and Security, London, UK, Jun. 2006, pp. 511-525.

[Bur09] R. Burkholder, "Electromagnetic models for exploiting multi-path propagation in through-wall radar imaging," in International Conference on Electromagnetics in Advanced Applications, Torino, Italy, Sep. 2009, pp. $572-575$.

[BV04] S. Boyd and L. Vandenberghe, Convex Optimization. New York, NY: Cambridge University Press, Mar. 2004. 
[CD94] S. Chen and D. Donoho, "Basis pursuit," in Conference Record of the Twenty-Eighth Asilomar Conference on Signals, Systems and Computers, vol. 1, Oct. 1994, pp. 41-44.

[CDS01] S. Chen, D. Donoho, and M. Saunders, "Atomic decomposition by basis pursuit," SIAM Review, vol. 43, no. 1, pp. 129-159, 2001.

[Cha11] P. C. Chang, "Physics-based inverse processing and multi-path exploitation for through-wall radar imaging," Ph.D. dissertation, Ohio State University, 2011.

[Che14] S. Chen, "Another particle swarm toolbox (http://www.mathworks.com/ matlabcentral/fileexchange/25986)," MATLAB Central File Exchange, 2009-14, retrieved April 1st 2014.

[CM91] J. C. Curlander and R. N. McDonough, Synthetic aperture radar. New York, NY: John Wiley and Sons, 1991.

[CRT06] E. Candès, J. Romberg, and T. Tao, "Stable signal recovery from incomplete and inaccurate measurements," Communications on Pure and Applied Mathematics, vol. 59, no. 8, p. 1207, 2006.

[CSD14] B. Chan, P. Sévigny, and D. D. J. DiFilippo, "Experimental 3-D SAR human target signature analysis," in Proc. SPIE 9243, SAR Image Analysis, Modeling, and Techniques XIV, vol. 9243, 2014, p. 92430Z.

[CT05] E. Candès and T. Tao, "Decoding by linear programming," IEEE Transactions on Information Theory, vol. 51, no. 12, pp. 4203-4215, Dec. 2005.

[CT06] E. Candès and T. Tao, "Near-optimal signal recovery from random projections: Universal encoding strategies?" IEEE Transactions on Information Theory, vol. 52, no. 12, pp. 5406 -5425, Dec. 2006.

[CW08] E. Candès and M. Wakin, "An introduction to compressive sampling," IEEE Signal Processing Magazine, vol. 25, no. 2, pp. 21-30, 2008.

[DA89] J. Davis and A. Annan, "Ground-penetrating radar for high-resolution mapping of soil and rock stratigraphy," Geophysical prospecting, vol. 37, no. 5, pp. 531-551, 1989.

[Dan96] D. Daniels, "Surface-penetrating radar," Electronics Communication Engineering Journal, vol. 8, no. 4, pp. 165-182, Aug. 1996.

[DAZ09] C. Debes, M. G. Amin, and A. Zoubir, "Target detection in single- and multiple-view through-the-wall radar imaging," IEEE Transactions on Geoscience and Remote Sensing, vol. 47, no. 5, pp. 1349-1361, 2009.

[DE03] D. Donoho and M. Elad, "Optimally sparse representation in general (nonorthogonal) dictionaries via $\ell_{1}$ minimization," Proceedings of the $\mathrm{Na}$ tional Academy of Sciences, vol. 100, no. 5, pp. 2197-2202, 2003. 
[Deb10] C. Debes, "Advances in detection and classification for through-the-wall radar imaging," Ph.D. dissertation, Technische Unitersität Darmstadt, 2010.

[DET06] D. Donoho, M. Elad, and V. Temlyakov, "Stable recovery of sparse overcomplete representations in the presence of noise," IEEE Transactions on Information Theory, vol. 52, no. 1, pp. 6-18, Jan. 2006.

[DH01] D. Donoho and X. Huo, "Uncertainty principles and ideal atomic decomposition," IEEE Transactions on Information Theory, vol. 47, no. 7, pp. 2845-2862, 2001.

[DK14] V. Dang and O. Kilic, "Joint DoA-range-doppler tracking of moving targets based on compressive sensing," in IEEE International Symposium on Antennas and Propagation and USNC-URSI Radio Science Meeting, Memphis, TN, Jul. 2014, pp. 141-142.

[DL09] T. Dogaru and C. Le, "SAR images of rooms and buildings based on FDTD computer models," IEEE Transactions on Geoscience and Remote Sensing, vol. 47, no. 5, pp. 1388-1401, May 2009.

[DM09] W. Dai and O. Milenkovic, "Subspace pursuit for compressive sensing signal reconstruction," IEEE Transactions on Information Theory, vol. 55, no. 5, pp. 2230-2249, May 2009.

[DR95] A. Dutt and V. Rokhlin, "Fast Fourier transforms for nonequispaced data, II," Applied and Computational Harmonic Analysis, vol. 2, no. 1, pp. 85100, 1995.

[DS08] M. Dehmollaian and K. Sarabandi, "Refocusing through building walls using synthetic aperture radar," IEEE Transactions on Geoscience and Remote Sensing, vol. 46, no. 6, pp. 1589-1599, Jun. 2008.

[DWZA10] C. Debes, C. Weiss, A. Zoubir, and M. G. Amin, "Wall-clutter mitigation using cross-beamforming in through-the-wall radar imaging," in European Signal Processing Conference (EUSIPCO), Aalborg, Denmark, Aug. 2010.

[DYZ11] W. Deng, W. Yin, and Y. Zhang, "Group sparse optimization by alternating direction method," Department of Computational and Applied Mathematics, Rice University, Technical Report TR11-06, 2011.

[EKB10] Y. C. Eldar, P. Kuppinger, and H. Bolcskei, "Block-sparse signals: Uncertainty relations and efficient recovery," IEEE Transactions on Signal Processing, vol. 58, no. 6, pp. 3042-3054, Jun. 2010.

[EM09] Y. C. Eldar and M. Mishali, "Robust recovery of signals from a structured union of subspaces," IEEE Transactions on Information Theory, vol. 55, no. 11, pp. 5302-5316, Nov. 2009. 
[FNW07] M. Figueiredo, R. Nowak, and S. Wright, "Gradient projection for sparse reconstruction: Application to compressed sensing and other inverse problems," IEEE Journal of Selected Topics in Signal Processing, vol. 1, no. 4, pp. 586-597, Dec. 2007.

[GCS13] G. Gennarelli, I. Catapano, and F. Soldovieri, "RF/microwave imaging of sparse targets in urban areas," IEEE Antennas and Wireless Propagation Letters, vol. 12, pp. 643-646, May 2013.

[GMS09a] A. Gurbuz, J. McClellan, and W. Scott, "A compressive sensing data acquisition and imaging method for stepped frequency GPRs," IEEE Transactions on Signal Processing, vol. 57, no. 7, pp. 2640-2650, Jul. 2009.

[GMS09b] A. Gurbuz, J. McClellan, and W. Scott, "Compressive sensing for subsurface imaging using ground penetrating radar," Signal Processing, vol. 89, no. 10, pp. 1959-1972, Oct. 2009.

[GMW81] P. E. Gill, W. Murray, and M. H. Wright, Practical optimization. London, UK: Academic Press, 1981.

[GRSS14] G. Gennarelli, G. Riccio, R. Solimene, and F. Soldovieri, "Radar imaging through a building corner," IEEE Transactions on Geoscience and Remote Sensing, vol. 52, no. 10, pp. 6750-6761, Oct. 2014.

[GS13] G. Gennarelli and F. Soldovieri, "A linear inverse scattering algorithm for radar imaging in multipath environments," IEEE Geoscience and Remote Sensing Letters, vol. 10, no. 5, pp. 1085-1089, Sep. 2013.

[GS14] G. Gennarelli and F. Soldovieri, "Radar imaging through cinderblock walls: Achievable performance by a model-corrected linear inverse scattering approach," IEEE Transactions on Geoscience and Remote Sensing, vol. 52, no. 10, pp. 6738-6749, Oct. 2014.

[HQWF10] Q. Huang, L. Qu, B. Wu, and G. Fang, "UWB through-wall imaging based on compressive sensing," IEEE Transactions on Geoscience and Remote Sensing, vol. 48, no. 3, pp. 1408-1415, Mar. 2010.

[JM09] Y. Jin and J. M. F. Moura, "Time-reversal detection using antenna arrays," IEEE Transactions on Signal Processing, vol. 57, no. 4, pp. 13961414, 2009.

[JRM10] J. Jackson, B. Rigling, and R. Moses, "Canonical scattering feature models for 3D and bistatic SAR," IEEE Transactions on Aerospace and Electronic Systems, vol. 46, no. 2, pp. 525-541, Apr. 2010.

[JXC08] S. Ji, Y. Xue, and L. Carin, "Bayesian compressive sensing," IEEE Transactions on Signal Processing, vol. 56, no. 6, pp. 2346-2356, 2008.

[KE95] J. Kennedy and R. Eberhart, "Particle swarm optimization," in Proceedings of IEEE International Conference on Neural Networks, vol. 4, Perth, Australia, Nov. 1995, pp. 1942-1948. 
$\left[\mathrm{KKL}^{+} 07\right] \quad$ S.-J. Kim, K. Koh, M. Lustig, S. Boyd, and D. Gorinevsky, "An interiorpoint method for large-scale $\ell 1$-regularized least squares," IEEE Journal of Selected Topics in Signal Processing, vol. 1, no. 4, pp. 606-617, 2007.

[KKT08] A. Karousos, G. Koutitas, and C. Tzaras, "Transmission and reflection coefficients in time-domain for a dielectric slab for UWB signals," in IEEE Vehicular Technology Conference, Singapore, May 2008, pp. 455-458.

[Kow09] B. Kowalski, M.and Torrésani, "Structured sparsity, from mixed norms to structured shrinkage," in Signal Processing with Adaptive Sparse Structured Representations (SPARS), Saint Malo, France, Apr. 2009.

[KSS11] S. Kidera, T. Sakamoto, and T. Sato, "Extended imaging algorithm based on aperture synthesis with double-scattered waves for UWB radars," IEEE Transactions on Geoscience and Remote Sensing, vol. 49, no. 12, pp. 51285139, Dec. 2011.

[LAAN12] E. Lagunas, M. G. Amin, F. Ahmad, and M. Nájar, "Wall mitigation techniques for indoor sensing within the cs framework," in IEEE Sensor Array and Multichannel Signal Processing Workshop (SAM), Hoboken, USA, Jun. 2012, pp. 213-216.

[LAAN13a] E. Lagunas, M. G. Amin, F. Ahmad, and M. Nájar, "Joint wall mitigation and compressive sensing for indoor image reconstruction," IEEE Transactions on Geoscience and Remote Sensing, vol. 51, no. 2, pp. 891-906, Feb. 2013.

[LAAN13b] E. Lagunas, M. G. Amin, F. Ahmad, and M. Nájar, "Determining building interior structures using compressive sensing," Journal of Electronic Imaging, vol. 22, no. 2, pp. 021 003-021 003, 2013.

[LAAZ13a] M. Leigsnering, F. Ahmad, M. G. Amin, and A. Zoubir, "Compressive sensing based specular multipath exploitation for through-the-wall radar imaging," in IEEE International Conference on Acoustics, Speech, and Signal Processing (ICASSP), Vancouver, Canada, May 2013, pp. 60046008 .

[LAAZ13b] M. Leigsnering, F. Ahmad, M. G. Amin, and A. Zoubir, "CS based wall ringing and reverberation mitigation for through-the-wall radar imaging," in IEEE Radar Conference (RADAR), Ottawa, Canada, Apr. 2013, pp. $1-5$.

[LAAZ13c] M. Leigsnering, F. Ahmad, M. G. Amin, and A. Zoubir, "General MIMO framework for multipath exploitation in through-the-wall radar imaging," in International Workshop on Compressed Sensing Applied to Radar (CoSeRa), Bonn, Germany, Sep. 2013.

[LAAZ14a] M. Leigsnering, F. Ahmad, M. G. Amin, and A. Zoubir, "CS based specular multipath exploitation in TWRI under wall position uncertainties," in IEEE Sensor Array and Multichannel Signal Processing Workshop (SAM), A Coruña, Spain, Jun. 2014, pp. 481-484. 
[LAAZ14b] M. Leigsnering, F. Ahmad, M. G. Amin, and A. Zoubir, "Multipath exploitation in through-the-wall radar imaging using sparse reconstruction," IEEE Transactions on Aerospace and Electronic Systems, vol. 50, no. 2, pp. 920-939, Apr. 2014.

[LAAZ14c] M. Leigsnering, F. Ahmad, M. G. Amin, and A. Zoubir, "Specular multipath exploitation for improved velocity estimation in through-the-wall radar imaging," in IEEE International Conference on Acoustics, Speech, and Signal Processing (ICASSP), Florence, Italy, May 2014, pp. 10601064.

[LAAZ14d] M. Leigsnering, M. G. Amin, F. Ahmad, and A. M. Zoubir, "Multipath exploitation and suppression for SAR imaging of building interiors: An overview of recent advances," IEEE Signal Processing Magazine, vol. 31, no. 4, pp. 110-119, Jul. 2014.

[LAAZ15a] M. Leigsnering, F. Ahmad, M. G. Amin, and A. Zoubir, "Compressive sensing based multipath exploitation for stationary and moving indoor target localization," IEEE Journal of Selected Topics in Signal Processing, vol. PP, no. 99, p. 1, Aug. 2015.

[LAAZ15b] M. Leigsnering, F. Ahmad, M. G. Amin, and A. Zoubir, "Multipath-aware velocity estimation for sparsity-based through-the-wall radar imaging," in IEEE International Radar Conference, Arlington, VA, May 2015.

[LAAZ15c] M. Leigsnering, F. Ahmad, M. G. Amin, and A. Zoubir, "Multipath exploitation in sparse scene recovery using sensing-through-wall distributed radar sensor configurations," in IEEE International Conference on Acoustics, Speech, and Signal Processing (ICASSP), Brisbane, Australia, Apr. 2015.

[LAAZ15d] M. Leigsnering, F. Ahmad, M. G. Amin, and A. Zoubir, "Parametric dictionary learning for sparsity-based TWRI in multipath environments," IEEE Transactions on Aerospace and Electronic Systems, 2015, accepted.

[LB15] G. Li and R. Burkholder, "Hybrid matching pursuit for distributed through-wall radar imaging," IEEE Transactions on Antennas and Propagation, vol. PP, no. 99, pp. 1-1, 2015.

$\left[\mathrm{LBM}^{+} 93\right]$ K. J. Langenberg, M. Brandfaß, K. Mayer, T. Kreutter, A. Brüll, P. Fellinger, and D. Huo, "Principles of microwave imaging and inverse scattering," EARSeL Advances in Remote Sensing, vol. 2, no. 1, pp. 163186, 1993.

[LDZ11] M. Leigsnering, C. Debes, and A. Zoubir, "Compressive sensing in through-the-wall radar imaging," in IEEE International Conference on Acoustics, Speech and Signal Processing (ICASSP), Prague, Czech Republic, May 2011, pp. 4008-4011. 
[LN10] C.-P. Lai and R. Narayanan, "Ultrawideband random noise radar design for through-wall surveillance," IEEE Transactions on Aerospace and Electronic Systems, vol. 46, no. 4, pp. 1716-1730, Oct. 2010.

[LZ11] M. Leigsnering and A. Zoubir, "Fast wideband near-field imaging using the non-equispaced FFT with application to through-wall radar," in European Signal Processing Conference (EUSIPCO), Barcelona, Spain, Aug. 2011, pp. 1708-1712.

[LZ14] M. Leigsnering and A. M. Zoubir, Compressive Sensing for Urban Radar. Boca Raton, FL: CRC Press, Aug. 2014, ch. Compressive Sensing for Urban Multipath Exploitation, pp. 153-196.

[LZG11] M. Leigsnering, A. Zoubir, and M. Ghogho, "Fast wideband near-field imaging with URAs applied to urban sensing," in IEEE International Workshop on Computational Advances in Multi-Sensor Adaptive Processing (CAMSAP), San Juan, Puerto Rico, Dec. 2011, pp. 73-76.

[LZL10] L. Li, W. Zhang, and F. Li, "A novel autofocusing approach for realtime through-wall imaging under unknown wall characteristics," IEEE Transactions on Geoscience and Remote Sensing, vol. 48, no. 1, pp. 423431, Jan. 2010.

$\left[\mathrm{MKA}^{+} 08\right]$ J. Moulton, S. Kassam, F. Ahmad, M. G. Amin, and K. Yemelyanov, "Target and change detection in synthetic aperture radar sensing of urban structures," in IEEE Radar Conference (RADAR), Rome, Italy, May 2008.

[ML13] H. Mansour and D. Liu, "Blind multi-path elimination by sparse inversion in through-the-wall-imaging," in IEEE International Workshop on Computational Advances in Multi-Sensor Adaptive Processing (CAMSAP), Saint Martin, Dec. 2013.

[NT10] D. Needell and J. A. Tropp, "CoSaMP: iterative signal recovery from incomplete and inaccurate samples," Communications of the ACM, vol. 53, no. 12, pp. 93-100, Dec. 2010.

[OSB99] A. V. Oppenheim, R. W. Schafer, and J. R. Buck, Discrete-time Signal Processing (2nd Ed.). Upper Saddle River, NJ: Prentice-Hall, Inc., 1999.

[PEPC10] L. Potter, E. Ertin, J. Parker, and M. Cetin, "Sparsity and compressed sensing in radar imaging," Proceedings of the IEEE, vol. 98, no. 6, pp. 1006-1020, Jun. 2010.

[PKB07] R. Poli, J. Kennedy, and T. Blackwell, "Particle swarm optimization," Swarm Intelligence, vol. 1, no. 1, pp. 33-57, Aug. 2007.

[PRK93] Y. Pati, R. Rezaiifar, and P. Krishnaprasad, "Orthogonal matching pursuit: recursive function approximation with applications to wavelet decomposition," in Conference Record of The Twenty-Seventh Asilomar Conference on Signals, Systems and Computers, vol. 1, Pacific Grove, CA, Nov. 1993, pp. 40-44. 
[PST01] D. Potts, G. Steidl, and M. Tasche, Fast Fourier transforms for nonequispaced data: A tutorial. Birkhäuser, 2001, ch. 12, pp. 247-269.

[PW09] O. Pele and M. Werman, "Fast and robust earth mover's distances," in IEEE 12th International Conference on Computer Vision, Kyoto, Japan, Sep. 2009, pp. 460-467.

[QAA13] J. Qian, F. Ahmad, and M. G. Amin, "Joint localization of stationary and moving targets behind walls using sparse scene recovery," Journal of Electronic Imaging, vol. 22, no. 2, p. 021002, Jun. 2013.

[RCP10] T. Ralston, G. Charvat, and J. Peabody, "Real-time through-wall imaging using an ultrawideband multiple-input multiple-output (MIMO) phased array radar system," in IEEE International Symposium on Phased Array Systems and Technology (ARRAY), Oct. 2010, pp. 551-558.

[Rom08] J. Romberg, "Imaging via compressive sampling," IEEE Signal Processing Magazine, vol. 25, no. 2, pp. 14-20, 2008.

[RSH10] M. A. Richards, J. A. Scheer, and W. A. Holm, Eds., Principles of Modern Radar: Basic Principles. Raleigh, NC: SciTech Publishing, 2010.

[RTG00] Y. Rubner, C. Tomasi, and L. J. Guibas, "The earth mover's distance as a metric for image retrieval," International Journal of Computer Vision, vol. 40, no. 2, pp. 99-121, Nov. 2000.

[SAA11] P. Setlur, M. G. Amin, and F. Ahmad, "Multipath model and exploitation in through-the-wall and urban radar sensing," IEEE Transactions on Geoscience and Remote Sensing, vol. 49, no. 10, pp. 4021-4034, Oct. 2011.

[SAN13] P. Setlur, G. Alli, and L. Nuzzo, "Multipath exploitation in through-wall radar imaging via point spread functions," IEEE Transactions on Image Processing, vol. 22, no. 12, pp. 4571-4586, Apr. 2013.

[SAS11] F. Soldovieri, F. Ahmad, and R. Solimene, "Validation of microwave tomographic inverse scattering approach via through-the-wall experiments in semicontrolled conditions," IEEE Geoscience and Remote Sensing Letters, vol. 8, no. 1, pp. 123-127, Jan. 2011.

[Sha49] C. Shannon, "Communication in the presence of noise," Proceedings of the IRE, vol. 37, no. 1, pp. 10-21, 1949.

[SLA $\left.{ }^{+} 15\right]$ M. Stiefel, M. Leigsnering, F. Ahmad, M. G. Amin, and A. M. Zoubir, "Distributed greedy sparse recovery for through-the-wall radar imaging," in International Review of Progress in Applied Computational Electromagnetics (ACES), vol. S07 Compressive Sensing, Williamsburg, VA, Mar. 2015 .

[SM12] G. Smith and B. Mobasseri, "Robust through-the-wall radar image classification using a target-model alignment procedure," IEEE Transactions on Image Processing, vol. 21, no. 2, pp. 754-767, Feb. 2012. 
[Sou99] M. Soumekh, Synthetic Aperture Radar Signal Processing with MATLAB Algorithms. New York, NY: John Wiley and Sons, 1999.

[SS07] F. Soldovieri and R. Solimene, "Through-wall imaging via a linear inverse scattering algorithm," IEEE Geoscience and Remote Sensing Letters, vol. 4, no. 4, pp. 513-517, Oct. 2007.

[SSA12] F. Soldovieri, R. Solimene, and F. Ahmad, "Sparse tomographic inverse scattering approach for through-the-wall radar imaging," IEEE Transactions on Instrumentation and Measurement, vol. 61, no. 12, pp. 3340-3350, 2012.

[SSPP09] R. Solimene, F. Soldovieri, G. Prisco, and R. Pierri, "Three-dimensional through-wall imaging under ambiguous wall parameters," IEEE Transactions on Geoscience and Remote Sensing, vol. 47, no. 5, pp. 1310-1317, 2009.

[SYL05] L.-P. Song, C. Yu, and Q. H. Liu, "Through-wall imaging (TWI) by radar: 2-D tomographic results and analyses," IEEE Transactions on Geoscience and Remote Sensing, vol. 43, no. 12, pp. 2793-2798, Dec. 2005.

[TBA11] F. H. C. Tivive, A. Bouzerdoum, and M. G. Amin, "An SVD-based approach for mitigating wall reflections in through-the-wall radar imaging," in IEEE Radar Conference (RADAR), Kansas City, MO, May 2011, pp. 519-524.

[TG07] J. Tropp and A. Gilbert, "Signal recovery from random measurements via orthogonal matching pursuit," IEEE Transactions on Information Theory, vol. 53, no. 12, pp. 4655-4666, Dec. 2007.

[THAD11] C. Thajudeen, A. Hoorfar, F. Ahmad, and T. Dogaru, "Measured complex permittivity of walls with different hydration levels and the effect on power estimation of TWRI target returns," Progress in Electromagnetic Research $B$, vol. 30, pp. 177-199, 2011.

[TP14] A. Tillmann and M. Pfetsch, "The computational complexity of the restricted isometry property, the nullspace property, and related concepts in compressed sensing," IEEE Transactions on Information Theory, vol. 60, no. 2, pp. 1248-1259, Feb. 2014.

[Tro04] J. Tropp, "Greed is good: algorithmic results for sparse approximation," IEEE Transactions on Information Theory, vol. 50, no. 10, pp. 2231-2242, Oct. 2004.

[TS10] Q. Tan and Y. Song, "A new method for multipath interference suppression in through-the-wall UWB radar imaging," in International Conference on Advanced Computer Control (ICACC), vol. 5, Shenyang, China, Mar. 2010, pp. 535-540.

[vRdW14] W. van Rossum and J. de Wit, Compressive Sensing for Urban Radar. Boca Raton, FL: CRC Press, Aug. 2014, ch. Overcomplete Dictionary Design for Sparse Reconstruction of Building Layout Mapping, pp. 49-86. 
[Wal91] G. K. Wallace, "The JPEG still picture compression standard," Communications of the ACM, vol. 34, no. 4, pp. 30-44, Apr. 1991.

[WF12] Y. Wang and A. Fathy, "Advanced system level simulation platform for three-dimensional UWB through-wall imaging SAR using time-domain approach," IEEE Transactions on Geoscience and Remote Sensing, vol. 50, no. 5, pp. 1986-2000, May 2012.

[WNF09] S. Wright, R. Nowak, and M. Figueiredo, "Sparse reconstruction by separable approximation," IEEE Transactions on Signal Processing, vol. 57, no. 7, pp. 2479-2493, Jul. 2009.

[WR04] D. Wipf and B. Rao, "Sparse bayesian learning for basis selection," IEEE Transactions on Signal Processing, vol. 52, no. 8, pp. 2153-2164, Aug. 2004.

[YA08] Y.-S. Yoon and M. Amin, "Compressed sensing technique for highresolution radar imaging," in Proceedings of SPIE Signal Processing, Sensor Fusion, and Target Recognition XVII, vol. 6968, no. 1, Orlando, FL, Mar. 2008, p. 69681A.

[YA09] Y.-S. Yoon and M. Amin, "Spatial filtering for wall-clutter mitigation in through-the-wall radar imaging," IEEE Transactions on Geoscience and Remote Sensing, vol. 47, no. 9, pp. 3192-3208, Sep. 2009.

[YL06] M. Yuan and Y. Lin, "Model selection and estimation in regression with grouped variables," Journal of the Royal Statistical Society, Series B, vol. 68, no. 1 , pp. 49-67, Dec. 2006.

[YLY13] L. Yuan, J. Liu, and J. Ye, "Efficient methods for overlapping group lasso," IEEE Transactions on Pattern Analysis and Machine Intelligence, vol. 35, no. 9, pp. 2104-2116, Jan. 2013.

$\left[\mathrm{ZAA}^{+} 12\right]$ W. Zhang, M. G. Amin, F. Ahmad, A. Hoorfar, and G. E. Smith, "Ultrawideband impulse radar through-the-wall imaging with compressive sensing," International Journal of Antennas and Propagation, vol. 2012, p. 11, 2012 .

[ZH11] W. Zhang and A. Hoorfar, "Two-dimensional diffraction tomographic algorithm for through-the-wall radar imaging," Progress in Electromagnetics Research B, vol. 31, pp. 205-218, 2011.

[ZHL10] W. Zhang, A. Hoorfar, and L. Li, "Through-the-wall target localization with time reversal music method," Progress in Electromagnetics Research, vol. 106, pp. 75-89, 2010.

[ZHTA11] W. Zhang, A. Hoorfar, C. Thajudeen, and F. Ahmad, "Full polarimetric beam-forming algorithm for through-the-wall radar imaging," Radio Science, vol. 46, p. RS0E16, Oct. 2011. 



\section{Curriculum Vitae}

Name:

Date of birth:

Place of birth:

\section{Education}

$10 / 2005-11 / 2010$

08/2008-05/2009

$05 / 2009$
Michael Leigsnering

18.07.1984

Karlsruhe

Technische Universität Darmstadt, Germany

Dipl.-Ing. Electrical Engineering and Information Tech.

Nanyang Technological University, Singapore

Integrated exchange program

Eichendorff-Gymnasium (College), Ettlingen, Germany High school degree (Abitur)

\section{Work experience}

$12 / 2010-04 / 2015$

Research associate

Signal Processing Group

Technische Universität Darmstadt, Germany

$03 / 2014-05 / 2014$

Visiting researcher

$03 / 2013-06 / 2013$

Radar Imaging Lab, Center for Advanced Communication,

$03 / 2012-05 / 2012$

Villanova University, Villanova, PA, USA

$11 / 2010-12 / 2010$

06/2009-09/2009

Visiting researcher

Visual and Audio Signal Processing Lab, ICT Research Institute, University of Wollongong, Wollongong, NSW, Australia

Internship

Robert Bosch GmbH, Stuttgart-Feuerbach, Germany 

Erklärung laut $\S 9$ der Promotionsordnung

Ich versichere hiermit, dass ich die vorliegende Dissertation allein und nur unter Verwendung der angegebenen Literatur verfasst habe. Die Arbeit hat bisher noch nicht zu Prüfungszwecken gedient.

Darmstadt, 1. Juni 2015, 
